Supporting Information

\title{
Au-Catalyzed Synthesis of (1Z-3E)-2-Pivaloxy-1,3-Dienes from Propargylic Pivalates
}

\author{
Guotao Li, Guozhu Zhang and Liming Zhang* \\ Department of Chemistry/216, University of Nevada, Reno \\ 1664 North Virginia Street, Reno, Nevada 89557
}

- Preparation of starting materials

page 2

- Gold-catalyzed synthesis of diene page 8

- Spectra .page 19 
General: Ethyl acetate (ACS grade), hexanes (ACS grade) and diethyl ether (ACS grade) were purchased from Fisher Scientific and used without further purification. Anhydrous tetrahydrofuran, anhydrous 1,2-dichloroethane and anhydrous dichloromethane in Pure-Pac ${ }^{\mathrm{TM}}$ from Aldrich were used directly without further treatment. Commercially available reagents were used without further purification. The NMR data were the same as reported. Reactions were monitored by thin layer chromatography (TLC) using silicycle precoated silica gel plates. Flash column chromatography was performed over silicycle silica gel (230-400 mesh). ${ }^{1} \mathrm{H}$ NMR and ${ }^{13} \mathrm{C}$ NMR spectra were recorded on a Varian $500 \mathrm{MHz}$ Unity plus spectrometer and a Varian $400 \mathrm{MHz}$ spectrometer using residue solvent peaks as internal standards. Infrared spectra were recorded with a Perkin Elmer FT-IR spectrum 2000 spectrometer and are reported in reciprocal centimeter $\left(\mathrm{cm}^{-1}\right)$. Mass spectra were recorded with Waters micromass ZQ detector using electron spray method.

\section{Preparation of Propargyl Isobutyrate 2c}

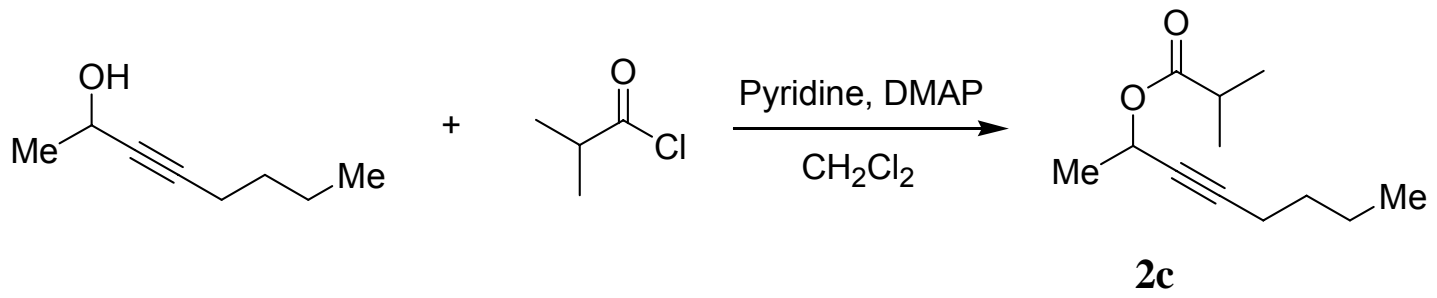

To a solution of a propargyl alcohol (1 eq.), pyridine (10 eq.) and DMAP (0.05 eq.) in anhydrous $\mathrm{CH}_{2} \mathrm{Cl}_{2}(0.5 \mathrm{M})$ was added isobutyryl chloride $\left(1.2\right.$ eq.) at $0^{\circ} \mathrm{C}$. The resulting mixture was stirred at room temperature for $2 \mathrm{~h}$. After all the propargyl alcohol was consumed, the mixture was quenched with methanol $(1 \mathrm{ml} / 1 \mathrm{mmol})$ to dissolve everything, and was stirred at room temperature for $1 \mathrm{~h}$. The reaction mixture was diluted with diethyl ether and extracted with water twice. The combined organic layers were washed with $1 \mathrm{M}$ aq. $\mathrm{HCl}$ twice, $1 \mathrm{M}$ aq. $\mathrm{NaOH}$ once and water once. The organic phase was dried over anhydrous $\mathrm{MgSO}_{4}$ and concentrated under vacuum to afford practically pure isobutyrate $2 \mathrm{c}$ in $73 \%$ yield. ${ }^{1} \mathrm{H}$ NMR $\left(500 \mathrm{MHz}, \mathrm{CDCl}_{3}\right) \delta: 5.45$ (tq, $\left.1 \mathrm{H}, \mathrm{J}=7.2 \mathrm{~Hz}\right), 2.54$ (heptet, $1 \mathrm{H}, \mathrm{J}=7.0 \mathrm{~Hz}$ ), 2.20 (dt, 2H, J = 7.0, $2.0 \mathrm{~Hz}$ ), 1.51-1.35 (m, 7H), 1.17 (dd, $6 \mathrm{H}, \mathrm{J}$ $=7.0,2.0 \mathrm{~Hz}$ ), 0.90 (t, $3 \mathrm{H}, \mathrm{J}=7.5 \mathrm{~Hz}$ ); ${ }^{13} \mathrm{C}$ NMR $\left(125 \mathrm{MHz}, \mathrm{CDCl}_{3}\right)$ ס: 176.1, 85.3, 78.7, $60.5,33.9,30.6,21.9,21.7,18.9,18.8,18.3,13.6$; IR (neat): 2962, 2937, 2876, 2249, 
$1739,1604,1470,1192,1153,1062 ; \mathrm{MS}\left(\mathrm{ES}^{+}\right)$Calculated for $\left[\mathrm{C}_{12} \mathrm{H}_{20} \mathrm{O}_{2} \mathrm{Na}\right]^{+}$219.2;

Found: 218.9 .

\section{General procedure A: Preparation of Propargyl Pivalate}

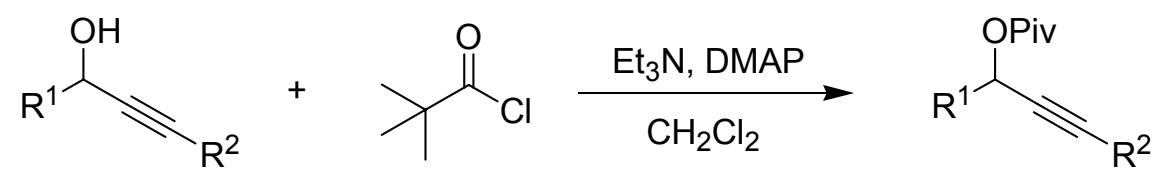

To a solution of a propargyl alcohol (1 eq.), $\mathrm{Et}_{3} \mathrm{~N}$ (10 eq.) and DMAP (0.05 eq.) in anhydrous $\mathrm{CH}_{2} \mathrm{Cl}_{2}(0.5 \mathrm{M})$ was added trimethylacetyl chloride $\left(1.2 \mathrm{eq}\right.$.) at $0^{\circ} \mathrm{C}$. The resulting mixture was stirred at room temperature for $2 \mathrm{~h}$. After all the propargyl alcohol was consumed, the mixture was quenched with methanol $(1 \mathrm{ml} / 1 \mathrm{mmol})$, and the resulting solution was stirred at room temperature for $1 \mathrm{~h}$. The reaction mixture was diluted with diethyl ether $(50 \mathrm{~mL})$ and washed with water $(2 \times 50 \mathrm{~mL}), 1 \mathrm{M}$ aq. $\mathrm{HCl}(2 \times 50 \mathrm{~mL}), 1 \mathrm{M}$ aq. $\mathrm{NaOH}(20 \mathrm{~mL})$ and water $(50 \mathrm{~mL})$. The organic phase was dried over anhydrous $\mathrm{MgSO}_{4}$ and concentrated under vacuum to yield practically pure pivaloate product. The propargyl pivalate was used for Au catalysis without further purification.

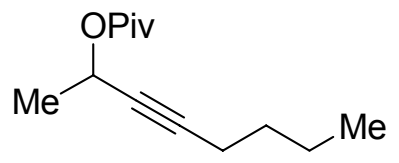

2d

Compound $\mathbf{2 d}$ was isolated in $93 \%$ yield following the general procedure A. ${ }^{1} \mathrm{H}$ NMR

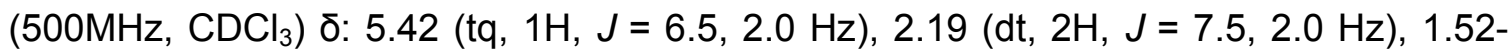
$1.36(\mathrm{~m}, 7 \mathrm{H}), 1.20(\mathrm{~s}, 9 \mathrm{H}), 0.90(\mathrm{t}, 3 \mathrm{H}, \mathrm{J}=7.0 \mathrm{~Hz}) ;{ }^{13} \mathrm{C} \mathrm{NMR}\left(100 \mathrm{MHz}, \mathrm{CDCl}_{3}\right)$ ठ: 177.3, 85.0, 78.8, 60.5, 38.5, 30.5, 27.0, 21.8, 21.6, 18.3, 13.5; IR (neat): 2962, 2937, 2875, $2250,1735,1459,1280,1149,1061$; $M S\left(E S^{+}\right)$Calculated for $\left[\mathrm{C}_{13} \mathrm{H}_{22} \mathrm{O}_{2} \mathrm{Na}\right]^{+}$233.2; Found: 232.7.

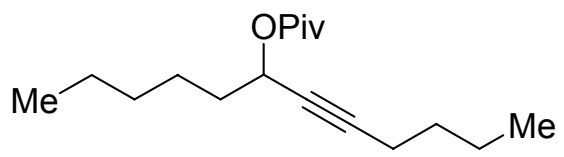

6a

Compound $\mathbf{6 a}$ was isolated in $90 \%$ yield following the general procedure A. ${ }^{1} \mathrm{H}$ NMR $\left(400 \mathrm{MHz}, \mathrm{CDCl}_{3}\right)$ ס: $5.32(\mathrm{tt}, 1 \mathrm{H}, J=6.4,2.0 \mathrm{~Hz}), 2.19(\mathrm{dt}, 2 \mathrm{H}, J=6.8,2.0 \mathrm{~Hz}), 1.75-$ 
$1.68(\mathrm{~m}, 2 \mathrm{H}), 1.51-1.28(\mathrm{~m}, 10 \mathrm{H}), 1.20(\mathrm{~s}, 9 \mathrm{H}), 0.92-0.87(\mathrm{~m}, 6 \mathrm{H}) ;{ }^{13} \mathrm{C} \mathrm{NMR}(125 \mathrm{MHz}$, $\left.\mathrm{CDCl}_{3}\right)$ ठ: 177.5, 85.6, 78.0, 64.3, 38.7, 35.0, 31.3, 30.6, 27.0, 24.7, 22.5, 21.8, 18.4, 13.9, 13.5; IR (neat): 2960, 2934, 2874, 2242, 1736, 1609, 1458, 1280, 1149, 1031; MS $\left(\mathrm{ES}^{+}\right)$Calculated for $\left[\mathrm{C}_{17} \mathrm{H}_{30} \mathrm{O}_{2} \mathrm{Na}\right]^{+}$289.2; Found: 289.0 .

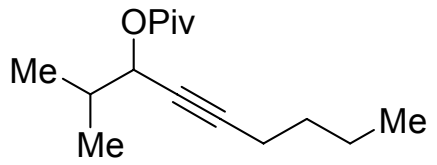

$6 b$

Compound $\mathbf{6 b}$ was isolated in $98 \%$ yield following the general procedure A. ${ }^{1} \mathrm{H}$ NMR $\left(500 \mathrm{MHz}, \mathrm{CDCl}_{3}\right)$ ס: 5.16 (dt, $1 \mathrm{H}, J=5.5,2.0 \mathrm{~Hz}$ ), 2.20 (dt, $2 \mathrm{H}, J=6.5,2.0 \mathrm{~Hz}$ ), 1.98$1.92(\mathrm{~m}, 1 \mathrm{H}), 1.51-1.36(\mathrm{~m}, 4 \mathrm{H}), 1.21(\mathrm{~s}, 9 \mathrm{H}), 1.00(\mathrm{~d}, \mathrm{~J}=6.5 \mathrm{~Hz}), 0.98(\mathrm{~d}, \mathrm{~J}=6.5 \mathrm{~Hz})$, 0.90 (t, $J=7.5 \mathrm{~Hz}) ;{ }^{13} \mathrm{C}$ NMR $\left(125 \mathrm{MHz}, \mathrm{CDCl}_{3}\right) \delta: 177.4,86.2,76.5,69.1,38.8,32.7$, 30.7, 27.1, 21.8, 18.3, 18.1, 17.7, 13.5; IR (neat): 2964, 2932, 2874, 1736, 1600, 1459, 1146; MS (ES ${ }^{+}$) Calculated for $\left[\mathrm{C}_{15} \mathrm{H}_{26} \mathrm{O}_{2} \mathrm{Na}\right]^{+}$261.2; Found: 260.9

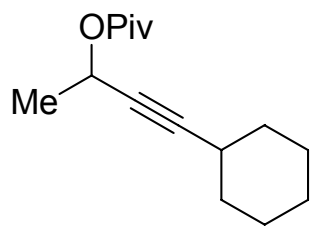

6c

Compound $\mathbf{6 c}$ was isolated in $81 \%$ yield following the general procedure A. ${ }^{1} \mathrm{H}$ NMR $\left(500 \mathrm{MHz}, \mathrm{CDCl}_{3}\right)$ ס: $5.44(\mathrm{dq}, 1 \mathrm{H}, \mathrm{J}=6.5,1.5 \mathrm{~Hz}), 2.40-2.36(\mathrm{~m}, 1 \mathrm{H}), 1.77-1.66(\mathrm{~m}, 4 \mathrm{H})$, 1.57-1.39 (m, 5H), 1.35-1.25 (m, 4H), $\left.1.20(\mathrm{~s}, 9 \mathrm{H}) ;{ }^{13} \mathrm{C} \mathrm{NMR} \mathrm{(100} \mathrm{MHz,} \mathrm{CDCl}_{3}\right)$ ס: 177.3, 88.9, 78.9, 60.5, 38.5, 32.4, 28.8, 26.9, 25.8, 24.6, 21.6; IR (neat): 2934, 2857, 1735, 1451, 1280, 1150, 1066; MS (ES $\left.{ }^{+}\right)$Calculated for $\left[\mathrm{C}_{15} \mathrm{H}_{24} \mathrm{O}_{2} \mathrm{Na}\right]^{+}$259.2; Found: 259.0.

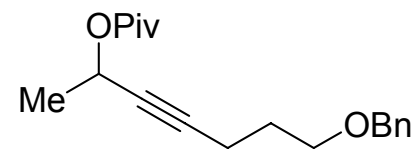

6d

Compound $6 \mathrm{~d}$ was isolated in $90 \%$ yield following the general procedure A. ${ }^{1} \mathrm{H}$ NMR $\left(400 \mathrm{MHz}, \mathrm{CDCl}_{3}\right)$ ס: 7.37-7.28 (m, 5H), 5.40 (tq, $\left.1 \mathrm{H}, \mathrm{J}=6.4,2.0 \mathrm{~Hz}\right), 4.51(\mathrm{~s}, 2 \mathrm{H}), 3.55(\mathrm{t}$, $2 \mathrm{H}, \mathrm{J}=6.4 \mathrm{~Hz}$ ), $2.33(\mathrm{dt}, 2 \mathrm{H}, \mathrm{J}=7.2,2.0 \mathrm{~Hz}$ ), 1.80 (quintet, $2 \mathrm{H}, \mathrm{J}=6.8 \mathrm{~Hz}$ ), $1.42(\mathrm{~d}, 3 \mathrm{H}$, 
$J=6.8 \mathrm{~Hz}), 1.20(\mathrm{~s}, 9 \mathrm{H}) ;{ }^{13} \mathrm{C} \mathrm{NMR}\left(125 \mathrm{MHz}, \mathrm{CDCl}_{3}\right) \delta: 177.4,138.5,128.4,127.6$, 127.5, 84.3, 79.2, 73.0, 68.7, 60.5, 38.6, 28.7, 27.0, 21.6, 15.5; IR (neat): 2936, 1730, $1608,1453,1280,1149$; $\mathrm{MS}\left(\mathrm{ES}^{+}\right)$Calculated for $\left[\mathrm{C}_{19} \mathrm{H}_{26} \mathrm{O}_{3} \mathrm{Na}\right]^{+} 325.2$; Found: 325.1.

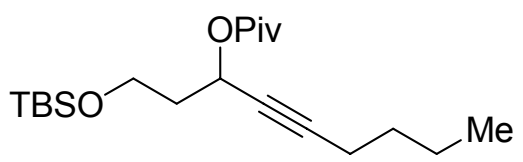

6h

Compound $\mathbf{6 h}$ was isolated in $80 \%$ yield following the general procedure A. ${ }^{1} \mathrm{H}$ NMR $\left(400 \mathrm{MHz}, \mathrm{CDCl}_{3}\right)$ ס: $5.44(\mathrm{tt}, 1 \mathrm{H}, \mathrm{J}=6.8,1.6 \mathrm{~Hz}), 3.78-3.67(\mathrm{~m}, 2 \mathrm{H}), 2.19(\mathrm{dt}, 2 \mathrm{H}, \mathrm{J}=7.2$, 2.0 Hz), 2.04-1.88 (m, 2H), 1.51-1.32 (m, 4H), $1.20(\mathrm{~s}, 9 \mathrm{H}), 0.90-0.88(\mathrm{~m}, 12 \mathrm{H}), 0.05(\mathrm{~s}$, 3H), 0.04 (s, 3H); ${ }^{13} \mathrm{C} \mathrm{NMR} \mathrm{(100} \mathrm{MHz,} \mathrm{CDCl}_{3}$ ) $\delta: 177.2,85.8,77.7,61.5,59.0,38.7,38.1$, 30.6, 27.0, 25.9, 21.8, 18.4, 18.3, 13.6, -5.4, -5.4; IR (neat): 2960, 2932, 2860, 1738, 1596, 1464, 1280, 1257, 1148, 1107, 957, 837; MS $\left(\mathrm{ES}^{+}\right)$Calculated for $\left[\mathrm{C}_{20} \mathrm{H}_{38} \mathrm{O}_{3} \mathrm{SiNa}\right]^{+}$ 377.3; Found: 377.1.

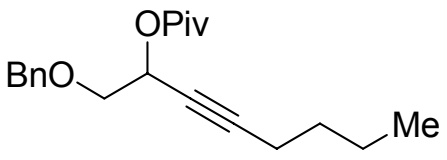

6i

Compound $6 \mathbf{i}$ was isolated in $81 \%$ yield following the general procedure A. ${ }^{1} \mathrm{H}$ NMR

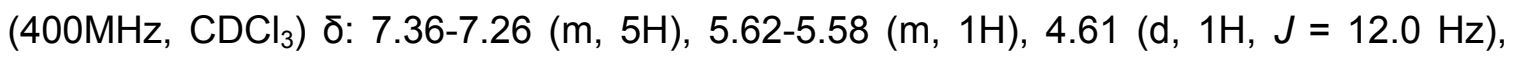
$4.56(\mathrm{~d}, 1 \mathrm{H}, \mathrm{J}=12.0 \mathrm{~Hz}), 3.71-3.63(\mathrm{~m}, 2 \mathrm{H}), 2.19(\mathrm{dt}, 2 \mathrm{H}, \mathrm{J}=6.8,2.0 \mathrm{~Hz}), 1.51-1.33(\mathrm{~m}$, $4 \mathrm{H}), 1.22(\mathrm{~s}, 9 \mathrm{H}), 0.89$ (t, 3H, J = 7.2 Hz); ${ }^{13} \mathrm{C} \mathrm{NMR}\left(100 \mathrm{MHz}, \mathrm{CDCl}_{3}\right) \delta: 177.4,137.9$, 128.4, 127.7, 127.6, 87.0, 75.1, 73.0, 71.6, 63.1, 38.1, 38.7, 30.4, 27.0, 21.8, 18.4, 13.5; IR (neat): 2961, 2934, 2873, 1737, 1600, 1457, 1279, 1147, 1042; MS (ES $\left.{ }^{+}\right)$Calculated for $\left[\mathrm{C}_{20} \mathrm{H}_{28} \mathrm{O}_{3} \mathrm{Na}\right]^{+}$339.2; Found: 339.1 .

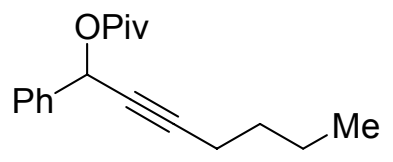

$6 \mathbf{k}$

Compound $\mathbf{6 k}$ was isolated in $99 \%$ yield following the general procedure A. ${ }^{1} \mathrm{H}$ NMR $\left(500 \mathrm{MHz}, \mathrm{CDCl}_{3}\right)$ ס: 7.50-7.48 (m, 2H), 7.38-7.31 (m, 3H), $6.43(\mathrm{t}, 1 \mathrm{H}, \mathrm{J}=2.0 \mathrm{~Hz}), 2,26$ (dt, $2 \mathrm{H}, J=7.0,2.0 \mathrm{~Hz}), 1.54-1.49(\mathrm{~m}, 2 \mathrm{H}), 1.45-1.37(\mathrm{~m}, 2 \mathrm{H}), 1.21(\mathrm{~s}, 9 \mathrm{H}), 0.91(\mathrm{t}, 3 \mathrm{H}, \mathrm{J}$ 
$=7.5 \mathrm{~Hz}) ;{ }^{13} \mathrm{C}$ NMR $\left(100 \mathrm{MHz}, \mathrm{CDCl}_{3}\right)$ ס: 177.3, 138.0, 128.4, 127.3, 87.9, 76.9, 65.7, 38.7, 30.5, 27.0, 21.9, 18.5, 13.6; IR (neat): 2961, 2932, 2874, 1737, 1607, 1457, 1272, 1136, 1031; MS (ES $\left.{ }^{+}\right)$Calculated for $\left[\mathrm{C}_{18} \mathrm{H}_{24} \mathrm{O}_{2} \mathrm{Na}\right]^{+}$295.2; Found: 294.9 .

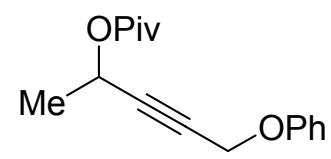

6l

Compound $6 \mathrm{I}$ was isolated in $95 \%$ yield following the general procedure A. ${ }^{1} \mathrm{H}$ NMR $\left(400 \mathrm{MHz}, \mathrm{CDCl}_{3}\right)$ ס: 7.32-7.26 (m, 3H), 7.00-6.95 (m, 2H), 5.46 (tq, $1 \mathrm{H}, J=6.4,1.6 \mathrm{~Hz}$ ), $4.71(\mathrm{~d}, 2 \mathrm{H}, \mathrm{J}=1.6 \mathrm{~Hz}), 1.46(\mathrm{~d}, 3 \mathrm{H}, \mathrm{J}=6.8 \mathrm{~Hz}), 1.19(\mathrm{~s}, 9 \mathrm{H}) ;{ }^{13} \mathrm{C} \mathrm{NMR}(125 \mathrm{MHz}$, $\mathrm{CDCl}_{3}$ ) ס: 177.3, 157,7, 129.4, 121.5, 115.0, 85.9, 79.3, 60.0, 56.1, 38.6, 27.0, 21.0; IR (neat): 2975, 2935, 2871, 1734, 1600, 1496, 1216, 1145, 1033; MS (ES ${ }^{+}$) Calculated for $\left[\mathrm{C}_{16} \mathrm{H}_{30} \mathrm{O}_{3} \mathrm{Na}\right]^{+}$283.2; Found: 283.0.

\section{Preparation of $6 e$}
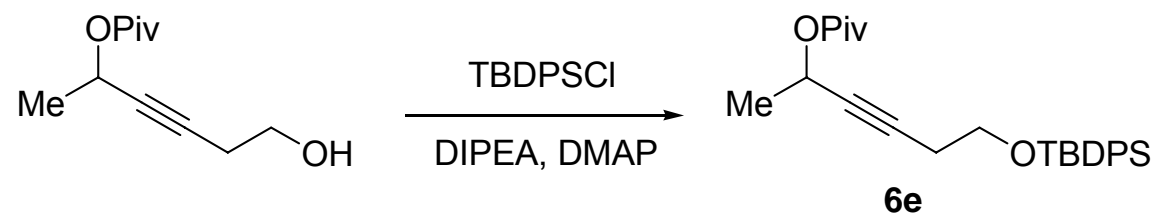

To a solution of the alcohol (2.5 mmol) and DIPEA (3 mmol, $1.2 \mathrm{eq})$ in anhydrous $\mathrm{CH}_{2} \mathrm{Cl}_{2}$ $(10 \mathrm{~mL})$ was added catalytic amount DMAP. The solution was cooled in an ice-water bath and treated with ${ }^{t}$ Butyldiphenylsilyl chloride $(2.75 \mathrm{mmol}, 1.1 \mathrm{eq})$. The ice-water bath was removed, and the reaction was stirred at room temperature for $1 \mathrm{~h}$. The reaction mixture was then diluted with $\mathrm{CH}_{2} \mathrm{Cl}_{2}(20 \mathrm{~mL})$ and washed with water $(3 \times 20 \mathrm{~mL})$ and brine $\left(20 \mathrm{~mL}\right.$ ). The combined organic layers were dried (anhydrous $\mathrm{MgSO}_{4}$ ), filtered, and concentrated. The residue was purified through silica gel flash column chromatography (eluents: EtOAc and hexanes $=10: 1)$ to yield the desired product $(2 \mathrm{mmol}$, yield $80 \%)$. ${ }^{1} \mathrm{H}$ NMR $\left(500 \mathrm{MHz}, \mathrm{CDCl}_{3}\right)$ ס: $7.68(\mathrm{~d}, 4 \mathrm{H}, \mathrm{J}=7.0 \mathrm{~Hz}), 7.44-7.37(\mathrm{~m}, 6 \mathrm{H}), 5.41(\mathrm{tq}, 1 \mathrm{H}, \mathrm{J}$ $=6.5,2.0 \mathrm{~Hz}), 3.75(\mathrm{t}, 2 \mathrm{H}, \mathrm{J}=7.0 \mathrm{~Hz}), 2.62(\mathrm{dt}, 2 \mathrm{H}, \mathrm{J}=7.0,2.0 \mathrm{~Hz}), 1.42(\mathrm{~d}, 3 \mathrm{H}, \mathrm{J}=7.0$

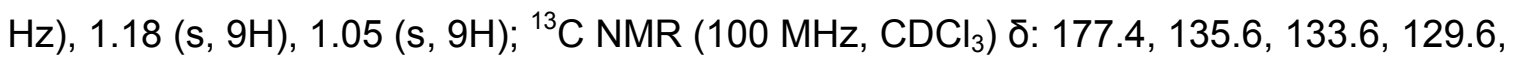
127.7, 82.1, 79.8, 62.2, 60.4, 38.6, 27.0, 26.7, 22.9, 21.6, 19.2; IR (neat): 3072, 2933, 2858, 1733, 1474, 1279, 1151; MS (ES ${ }^{+}$Calculated for $\left[\mathrm{C}_{27} \mathrm{H}_{36} \mathrm{NaO} \mathrm{Si}\right]^{+}$459.2; Found: 459.1 


\section{General procedure B: Preparation of $6 f$ and $6 g$}

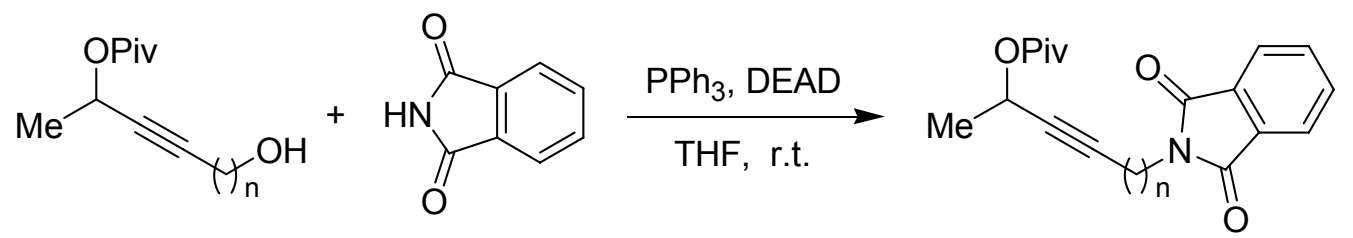

To a solution of the hydroxyl pivalate (1 eq.), phthalimide (1.1 eq.), $\mathrm{PPh}_{3}$ (1.1 eq.) in anhydrous THF (0.3M) were added DEAD (1.1 eq.) dropwise at $0^{\circ} \mathrm{C}$. The resulting mixture was stirred at room temperature for $2 \mathrm{~h}$. After all the hydroxyl pivalate was consumed, the mixture was concentrated under vacuum, and the residue was purified via silica gel flash column chromatography (eluents: hexanes/ethyl acetate $=3: 1$ ) to yield the desired product.

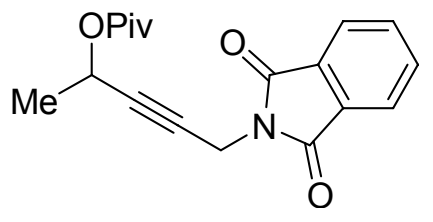

6f

Compound $6 \mathrm{f}$ was isolated in $80 \%$ yield following the general procedure $\mathrm{B} .{ }^{1} \mathrm{H}$ NMR

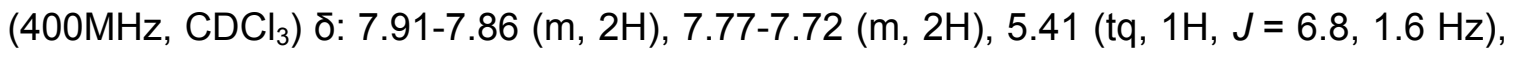
$4.48(\mathrm{~d}, 2 \mathrm{H}, \mathrm{J}=1.6 \mathrm{~Hz}), 1.43(\mathrm{~d}, 3 \mathrm{H}, \mathrm{J}=6.8 \mathrm{~Hz}), 1.18(\mathrm{~s}, 9 \mathrm{H}) ;{ }^{13} \mathrm{C} \mathrm{NMR}(125 \mathrm{MHz}$, $\mathrm{CDCl}_{3}$ ) ঠ: 177.3, 167.0, 134.2, 132.1, 123.5, 81.7, 77.9, 59.9, 38.6, 27.2, 27.0, 21.0; IR (neat): 2963, 1729, 1710, 1467, 1320, 1281, 1147, 1116, 1056, 939; MS $\left(\mathrm{ES}^{+}\right)$ Calculated for $\left[\mathrm{C}_{18} \mathrm{H}_{19} \mathrm{NO}_{4} \mathrm{Na}\right]^{+} 336.1$; Found: 336.0

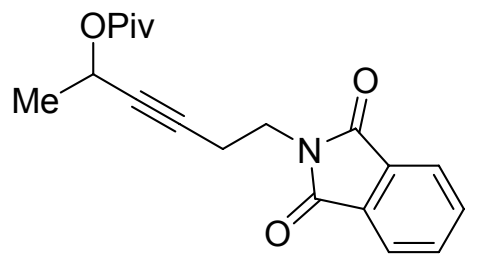

$6 \mathrm{~g}$

Compound $6 \mathrm{~g}$ was isolated in $85 \%$ yield following the general procedure $\mathrm{B} .{ }^{1} \mathrm{H}$ NMR

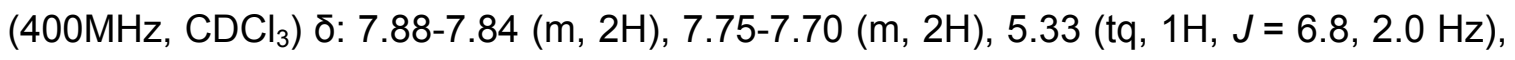
$3.86(\mathrm{t}, 2 \mathrm{H}, \mathrm{J}=7.2 \mathrm{~Hz}$ ), 2.62 (dt, $2 \mathrm{H}, \mathrm{J}=7.2,2.0 \mathrm{~Hz}$ ), 1.37 (d, 3H, J = 6.4 Hz), 1.15 (s, $9 \mathrm{H}) ;{ }^{13} \mathrm{C}$ NMR $\left(100 \mathrm{MHz}, \mathrm{CDCl}_{3}\right) \delta: 177.3,168.0,134.0,132.0,123.3,80.8,80.7,60.2$, 
38.5, 36.5, 26.9, 21.3, 18.6; IR (neat): 2980, 1775, 1720, 1616, 1438, 1396, 1365, 1150, 1000; $\mathrm{MS}\left(\mathrm{ES}^{+}\right)$Calculated for $\left[\mathrm{C}_{19} \mathrm{H}_{21} \mathrm{NO}_{4} \mathrm{Na}\right]^{+} 350.1$; Found: 350.0

\section{Preparation of $6 \mathbf{j}$}

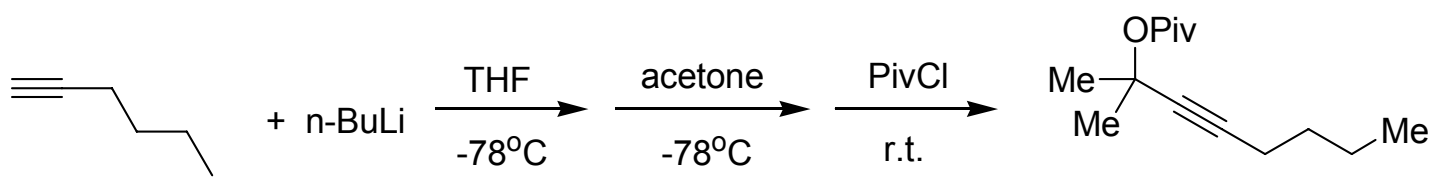

6j

To a solution of the 1-hexyne $(4.3 \mathrm{~mL}, 37.5 \mathrm{mmol})$ in anhydrous THF (25 mL) was added $n$-BuLi (1.6 M in hexane, $23 \mathrm{~mL}, 36 \mathrm{mmol})$ at $-78^{\circ} \mathrm{C}$. 30 minutes later, anhydrous acetone was added in dropwise at $-78^{\circ} \mathrm{C}$. The reaction mixture was stirred at $-78^{\circ} \mathrm{C}$ for $1 \mathrm{~h}$ before the addition of trimethylacetyl chloride $(4.2 \mathrm{~mL}, 40 \mathrm{mmol})$. The reaction mixture was allowed to rise to room temperature. Stirring was continued for 1.5 hours. The reaction mixture was then washed with water $(20 \mathrm{~mL})$ and brine $(20 \mathrm{~mL})$. The organic layer were dried (anhydrous $\mathrm{MgSO}_{4}$ ), filtered, and concentrated. The residue was purified through silica gel flash column chromatography (eluents: EtOAc and hexanes $=5: 1)$ to yield the desired ester $(19 \mathrm{mmol}$, yield $50 \%)$. ${ }^{1} \mathrm{H} \mathrm{NMR}(400 \mathrm{MHz}$, $\left.\mathrm{CDCl}_{3}\right)$ ס: $2.19(\mathrm{t}, 2 \mathrm{H}, \mathrm{J}=7.2 \mathrm{~Hz}), 1.62(\mathrm{~s}, 6 \mathrm{H}), 1.50-1.33(\mathrm{~m}, 4 \mathrm{H}), 1.17(\mathrm{~s}, 9 \mathrm{H}), 0.90(\mathrm{t}$, $2 \mathrm{H}, \mathrm{J}=7.2 \mathrm{~Hz}) ;{ }^{13} \mathrm{C}$ NMR $\left(125 \mathrm{MHz}, \mathrm{CDCl}_{3}\right) \delta: 176.7,84.1,81.5,72.1,39.0,30.7,29.2$, 27.0, 21.8, 18.3, 13.6; IR (neat): 2961, 2936, 2875, 2246, 1739, 1608, 1480, 1460, 1286, 1265, 1125; MS $\left(\mathrm{ES}^{+}\right)$Calculated for $\left[\mathrm{C}_{14} \mathrm{H}_{24} \mathrm{O}_{2} \mathrm{Na}\right]^{+}$247.2; Found: 247.0.

\section{General procedure C: IPrAuNTf 2 -catalyzed reaction of propargylic pivalate}

To a $0.5 \mathrm{M}$ solution of a propargyl pivalate $(1 \mathrm{~mol})$ in anhydrous 1,2-dichloroethane (2 $\mathrm{mL}$ ) in a flame-dried 2-dram via was added $5 \mathrm{~mol} \%$ of IPrAuNTf $\mathrm{PL}_{2}$. The vial was sealed tightly with Teflon tape inside and outside the cap and heated up to $80^{\circ} \mathrm{C}$ for the indicated time. Upon the completion of the reaction, the solvent was removed under vacuum, and the residue was purified via basified silica gel $\left(E_{3} N\right)$ flash column chromatography (eluents: hexanes) to yield the desired 2-pivaloxy-1,3-dienes.

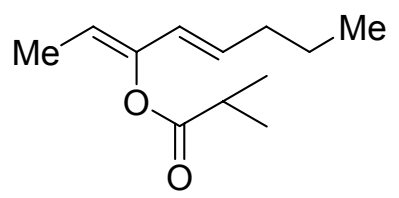


${ }^{1} \mathrm{H}$ NMR $\left(400 \mathrm{MHz}, \mathrm{CDCl}_{3}\right)$ ס: $5.93(\mathrm{dt}, 1 \mathrm{H}, \mathrm{J}=15.6,1.2 \mathrm{~Hz}), 5.55(\mathrm{dt}, 1 \mathrm{H}, \mathrm{J}=15.6,7.2$ $\mathrm{Hz}$ ), 5.27 (q, $1 \mathrm{H}, J=7.2 \mathrm{~Hz}$ ), 2.78 (heptet, $1 \mathrm{H}, J=7.2 \mathrm{~Hz}$ ), 2.05 (q, 2H, J = 7.2 Hz), 1.56 (d, $3 \mathrm{H}, J=7.2 \mathrm{~Hz}$ ), 1.40 (sextet, $2 \mathrm{H}, J=7.2 \mathrm{~Hz}$ ), 1.31 (d, 6H, J = $7.2 \mathrm{~Hz}), 0.89(\mathrm{t}, 3 \mathrm{H}, \mathrm{J}=$ $7.2 \mathrm{~Hz}) ;{ }^{13} \mathrm{C}$ NMR $\left(100 \mathrm{MHz}, \mathrm{CDCl}_{3}\right) \delta: 174.1,146.3,129.0,124.7,114.0,34.2,34.1$, 22.2, 19.1, 13.6, 11.0; IR (neat): 2965, 2933, 2875, 1756, 1471, 1387, 1136, 958; MS $\left(\mathrm{ES}^{+}\right)$Calculated for $\left[\mathrm{C}_{12} \mathrm{H}_{20} \mathrm{O}_{2} \mathrm{Na}\right]^{+}$219.2; Found: 218.9 .

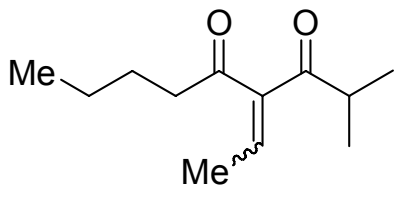

$5 c$

Major: ${ }^{1} \mathrm{H}$ NMR $\left(400 \mathrm{MHz}, \mathrm{CDCl}_{3}\right.$ ) $\delta: 6.82(\mathrm{q}, 1 \mathrm{H}, \mathrm{J}=7.2 \mathrm{~Hz}$ ), 2.83 (heptet, $1 \mathrm{H}, \mathrm{J}=7.2$ $\mathrm{Hz}), 2.62(\mathrm{t}, 2 \mathrm{H}, \mathrm{J}=7.6 \mathrm{~Hz}), 1.86(\mathrm{~d}, 3 \mathrm{H}, \mathrm{J}=6.8 \mathrm{~Hz}), 1.64-1.56(\mathrm{~m}, 2 \mathrm{H}), 1.39-1.31(\mathrm{~m}$, $2 \mathrm{H}), 1.11(\mathrm{~d}, 6 \mathrm{H}, \mathrm{J}=7.2 \mathrm{~Hz}), 0.92(\mathrm{t}, 3 \mathrm{H}, \mathrm{J}=7.2 \mathrm{~Hz}) ;{ }^{13} \mathrm{C}$ NMR $\left(125 \mathrm{MHz}, \mathrm{CDCl}_{3}\right) \delta$ : 210.0, 199.6, 145.6, 140.4, 41.1, 37.6, 26.4, 22.4, 17.6, 15.7, 13.9; IR (neat): 2963, 2932, $2871,1699,1663,1460,1122,1091,1051 ; \mathrm{MS}^{\left(E S^{+}\right)}$Calculated for $\left[\mathrm{C}_{12} \mathrm{H}_{20} \mathrm{O}_{2} \mathrm{Na}\right]^{+}$ 219.2; Found: 218.9.

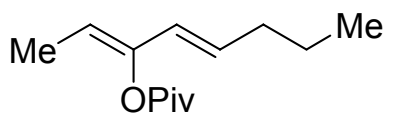

3d

Compound 3d was isolated in $83 \%$ yield following the general procedure $\mathrm{F}$. Reaction time: 9 h. ${ }^{1} \mathrm{H}$ NMR $\left(400 \mathrm{MHz}, \mathrm{CDCl}_{3}\right)$ ס: $5.93(\mathrm{dt}, 1 \mathrm{H}, \mathrm{J}=15.6,1.6 \mathrm{~Hz}), 5.55(\mathrm{dt}, 1 \mathrm{H}, \mathrm{J}=$ 15.6, $6.8 \mathrm{~Hz}), 5.26(\mathrm{q}, 1 \mathrm{H}, \mathrm{J}=7.2 \mathrm{~Hz}), 2.08-2.02(\mathrm{~m}, 2 \mathrm{H}), 1.55(\mathrm{~d}, 3 \mathrm{H}, \mathrm{J}=6.8 \mathrm{~Hz}), 1.45-$ $1.35(\mathrm{~m}, 11 \mathrm{H}), 0.89(\mathrm{t}, 3 \mathrm{H}, \mathrm{J}=7.6 \mathrm{~Hz}) ;{ }^{13} \mathrm{C}$ NMR $\left(125 \mathrm{MHz}, \mathrm{CDCl}_{3}\right) \delta: 175.5,146.4$, 128.9, 124.9, 114.0, 39.2, 34.2, 27.3, 22.3, 13.6, 10.9; IR (neat): 2962, 2933, 2874, 1752, 1481, 1459, 1273, 1128, 958; MS $\left(\mathrm{ES}^{+}\right)$Calculated for $\left[\mathrm{C}_{13} \mathrm{H}_{22} \mathrm{O}_{2} \mathrm{Na}\right]^{+}$233.2; Found: 232.8 .

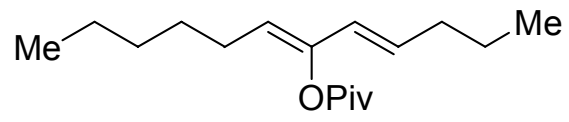

$7 a$

Compound $7 \mathrm{a}$ was isolated in $85 \%$ yield following the general procedure $\mathrm{F}$. Reaction time: 8 h. ${ }^{1} \mathrm{H}$ NMR $\left(400 \mathrm{MHz}, \mathrm{CDCl}_{3}\right)$ ס: $5.92(\mathrm{dt}, 1 \mathrm{H}, \mathrm{J}=15.6,1.2 \mathrm{~Hz}), 5.54(\mathrm{dt}, 1 \mathrm{H}, \mathrm{J}=$ 
15.2, 7.2 Hz), 5.20 (q, 1H, J = 7.6 Hz), 2.08-2.02 (m, 2H), $1.94(\mathrm{q}, 2 \mathrm{H}, \mathrm{J}=7.6 \mathrm{~Hz}), 1.45-$ $1.24(\mathrm{~m}, 17 \mathrm{H}), 0.91-0.86(\mathrm{~m}, 6 \mathrm{H}) ;{ }^{13} \mathrm{C}$ NMR $\left(125 \mathrm{MHz}, \mathrm{CDCl}_{3}\right) \delta: 175.6,145.5,129.0$, 124.9, 119.8, 39.1, 34.2, 31.5, 28.5, 27.3, 25.6, 22.4, 22.3, 13.9, 13.6; IR (neat): 2960, 2932, 2874, 1752, 1480, 1460, 1272, 1128, 958; MS $\left(E^{+}\right)$Calculated for $\left[\mathrm{C}_{17} \mathrm{H}_{30} \mathrm{O}_{2} \mathrm{Na}\right]^{+}$ 289.2; Found: 288.9 .

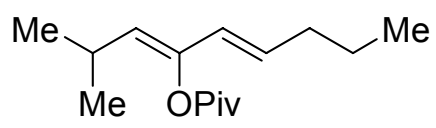

$7 \mathbf{b}$

Compound $\mathbf{7 b}$ was isolated in $75 \%$ yield following the general procedure $\mathrm{F}$. Reaction time: 10 h. ${ }^{1} \mathrm{H}$ NMR $\left(400 \mathrm{MHz}, \mathrm{CDCl}_{3}\right)$ ס: $5.87(\mathrm{dt}, 1 \mathrm{H}, \mathrm{J}=15.6,1.2 \mathrm{~Hz}), 5.50(\mathrm{dt}, 1 \mathrm{H}, \mathrm{J}=$ 15.6, 7.2 Hz), $5.01(\mathrm{~d}, 1 \mathrm{H}, \mathrm{J}=9.6 \mathrm{~Hz}), 2.41-2.32(\mathrm{~m}, 1 \mathrm{H}), 2.05-1.99(\mathrm{~m}, 2 \mathrm{H}), 1.46-1.27$ $(\mathrm{m}, 11 \mathrm{H}), 0.94(\mathrm{~d}, 6 \mathrm{H}, \mathrm{J}=7.2 \mathrm{~Hz}), 0.86(\mathrm{t}, 3 \mathrm{H}, \mathrm{J}=7.6 \mathrm{~Hz}) ;{ }^{13} \mathrm{C} \mathrm{NMR}\left(100 \mathrm{MHz}, \mathrm{CDCl}_{3}\right) \delta$ : $175.8,143.8,129.2,126.7,124.9,39.1,34.2,27.3,25.5,22.4,22.2,13.6$; IR (neat): 2961, 2933, 2873, 1752, 1635, 1460, 1272, 1128, 958; MS $\left(\mathrm{ES}^{+}\right)$Calculated for $\left[\mathrm{C}_{15} \mathrm{H}_{26} \mathrm{O}_{2} \mathrm{Na}\right]^{+}$261.2; Found: 261.1 .

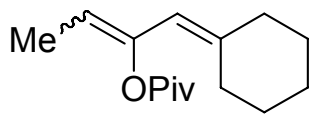

7c

Compound 7c was isolated in $80 \%$ yield following the general procedure $\mathrm{F}$. Reaction time: 10 h. $Z: E=8: 1 .{ }^{1} \mathrm{H} \mathrm{NMR}\left(400 \mathrm{MHz}, \mathrm{CDCl}_{3}\right) \delta: 5.57(\mathrm{~s}, 1 \mathrm{H}$, minor), $5.52(\mathrm{~s}, 1 \mathrm{H}$, major), 5.19-5.13 (m, 1H), 2.38-2.36 (m, 2H, major), 2.23-2.21 (m, 2H, minor), 2.17-2.09 $(\mathrm{m}, 2 \mathrm{H}), 1.62-1.54(\mathrm{~m}, 9 \mathrm{H}), 1.29$ (s, 9H, major), 1.23 (s, 9H, minor); ${ }^{13} \mathrm{C}$ NMR (100 MHz, $\mathrm{CDCl}_{3}$ ) ठ: 177.0, 175.9, 147.1, 145.8, 145.5, 144.4, 116.6, 114.2, 113.6, 113.3, 39.0, $38.6,37.4,36.8,30.6,29.9,28.4,27.7,27.4,27.2,27.1,26.4,26.3,12.5,10.7$; IR (neat): 2931, 2857, 1747, 1655, 1479, 1449, 1273, 1127, 1032, 982; MS (ES ${ }^{+}$) Calculated for $\left[\mathrm{C}_{15} \mathrm{H}_{24} \mathrm{O}_{2} \mathrm{Na}\right]^{+}$259.2; Found: 258.9 .

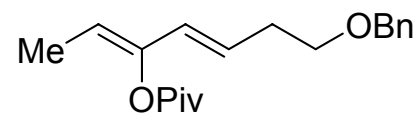

7d

Compound $\mathbf{7 d}$ was isolated in $67 \%$ yield following the general procedure $\mathrm{F}$. Reaction time: 10 h. ${ }^{1} \mathrm{H}$ NMR $\left(400 \mathrm{MHz}, \mathrm{CDCl}_{3}\right)$ ס: 7.36-7.25 (m, 5H), $6.00(\mathrm{~d}, 1 \mathrm{H}, \mathrm{J}=15.6 \mathrm{~Hz})$, 
$5.58(\mathrm{dt}, 1 \mathrm{H}, \mathrm{J}=15.6,7.2 \mathrm{~Hz}), 5.29(\mathrm{q}, 1 \mathrm{H}, \mathrm{J}=7.2 \mathrm{~Hz}), 4.5(\mathrm{~s}, 2 \mathrm{H}), 3.5(\mathrm{t}, 2 \mathrm{H}, J=6.8$ $\mathrm{Hz}$ ), 2.40 (q, $2 \mathrm{H}, \mathrm{J}=6.8 \mathrm{~Hz}$ ), 1.55 (d, 3H, J = 7.2 Hz), 1.34 (s, 9H); ${ }^{13} \mathrm{C}$ NMR $(100 \mathrm{MHz}$, $\mathrm{CDCl}_{3}$ ) $\delta:$ 175.5, 146.2, 138.4, 128.3, 127.5, 126.5, 124.9, 72.9, 69.5, 39.2, 32.6, 27.3, 11.0; IR (neat): 3035, 2975, 2862, 1749, 1635, 1480, 1456, 1273, 1127, 1030, 960; MS $\left(\mathrm{ES}^{+}\right)$Calculated for $\left[\mathrm{C}_{19} \mathrm{H}_{26} \mathrm{O}_{3} \mathrm{Na}\right]^{+}$325.2; Found: 325.1 .

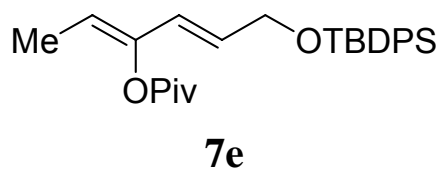

Compound $7 \mathrm{e}$ was isolated in $67 \%$ yield following the general procedure $\mathrm{F}$. Reaction time: 22 h. ${ }^{1} \mathrm{H}$ NMR $\left(400 \mathrm{MHz}, \mathrm{CDCl}_{3}\right)$ ס: 7.68-7.64 (m, 4H), 7.44-7.35 (m, 6H), 6.00 (dt, $1 \mathrm{H}, \mathrm{J}=15.6,1.6 \mathrm{~Hz}), 5.61(\mathrm{tt}, 1 \mathrm{H}, \mathrm{J}=15.6,4 \mathrm{~Hz}), 5.37(\mathrm{q}, 1 \mathrm{H}, \mathrm{J}=7.2 \mathrm{~Hz}), 4.26-4.24(\mathrm{~m}$, $2 \mathrm{H}), 1.58(\mathrm{~d}, 3 \mathrm{H}, \mathrm{J}=7.2 \mathrm{~Hz}), 1.33(\mathrm{~s}, 9 \mathrm{H}), 1.05(\mathrm{~s}, 9 \mathrm{H}) ;{ }^{13} \mathrm{C} \mathrm{NMR}\left(100 \mathrm{MHz}, \mathrm{CDCl}_{3}\right) \delta$ : 175.7, 146.4, 135.8, 133.8, 129.9, 127.9, 127.0, 124.5, 116.1, 77.5, 63.8, 39.4, 27.6, 27.0, 19.5, 11.4; IR (neat): 2933, 1748, 1590, 1462, 1276, 1114; MS (ES ${ }^{+}$) Calculated for [C27H36NaO3Si] ${ }^{+} 459.2$; Found: 459.1.

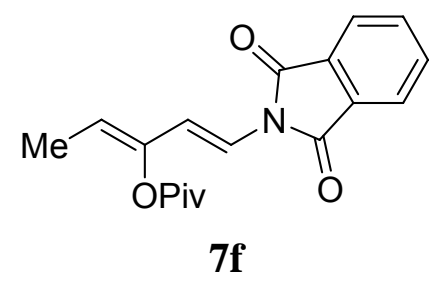

Compound $7 \mathrm{f}$ was isolated in $52 \%$ yield following the general procedure $\mathrm{F}$. Reaction time: 9 h. ${ }^{1} \mathrm{H}$ NMR $\left(400 \mathrm{MHz}, \mathrm{CDCl}_{3}\right)$ ס: 7.88-7.84 (m, 2H), 7.77-7.73 (m, 2H), 7.27 (d, $1 \mathrm{H}$, $J=14.8 \mathrm{~Hz}), 6.79(\mathrm{~d}, 1 \mathrm{H}, \mathrm{J}=14.8 \mathrm{~Hz}), 5.53(\mathrm{q}, 1 \mathrm{H}, \mathrm{J}=7.2 \mathrm{~Hz}), 1.63(\mathrm{~d}, 3 \mathrm{H}, \mathrm{J}=6.8 \mathrm{~Hz})$,

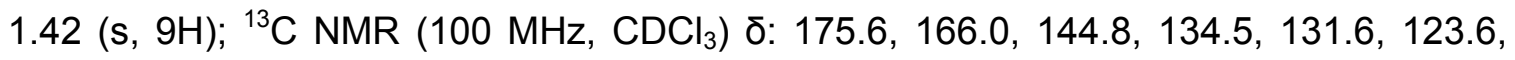
117.6, 115.9, 114.9, 39.3, 27.3, 11.4; IR (neat): 2976, 2925, 1746, 1724, 1612, 1456, 1388, 1115, 955; MS $\left(\mathrm{ES}^{+}\right)$Calculated for $\left[\mathrm{C}_{18} \mathrm{H}_{19} \mathrm{NO}_{4} \mathrm{Na}\right]^{+} 336.1$; Found: 335.9 .

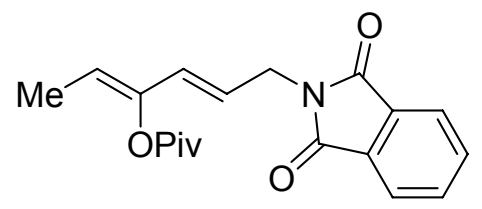

$7 \mathrm{~g}$ 
Compound $\mathbf{7 g}$ was isolated in $80 \%$ yield following the general procedure $\mathrm{F}$. Reaction time: 910 h. ${ }^{1} \mathrm{H}$ NMR $\left(400 \mathrm{MHz}, \mathrm{CDCl}_{3}\right)$ ס: 7.85-7.82 (m, 2H), 7.74-7.71 (m, 2H), $6.21(\mathrm{~d}$, $1 \mathrm{H}, \mathrm{J}=15.2 \mathrm{~Hz}$ ), $5.62(\mathrm{dt}, 1 \mathrm{H}, \mathrm{J}=15.2,6.8 \mathrm{~Hz}), 5.42(\mathrm{q}, 1 \mathrm{H}, \mathrm{J}=7.2 \mathrm{~Hz}), 4.30(\mathrm{~d}, 2 \mathrm{H}, \mathrm{J}$ $=6.8 \mathrm{~Hz}), 1.55(\mathrm{~d}, 3 \mathrm{H}, \mathrm{J}=6.8 \mathrm{~Hz}), 1.33(\mathrm{~s}, 9 \mathrm{H}) ;{ }^{13} \mathrm{C} \mathrm{NMR}\left(100 \mathrm{MHz}, \mathrm{CDCl}_{3}\right)$ ס: 175.3, $167.7,145.3,133.9,132.0,129.0,123.2,120.5,117.8,39.1,38.8,27.2,11.1$; IR (neat): 3060, 2988, 2988, 2908, 1772, 1744, 1713, 1430, 1392, 1258, 1129, 1111, 955; MS $\left(\mathrm{ES}^{+}\right)$Calculated for $\left[\mathrm{C}_{19} \mathrm{H}_{21} \mathrm{NO}_{4} \mathrm{Na}\right]^{+} 350.1$; Found: 350.0 .

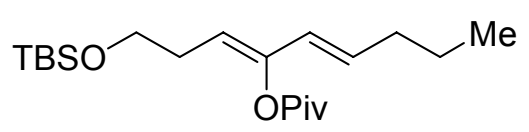

$7 \mathrm{~h}$

Compound $\mathbf{7 h}$ was isolated in $67 \%$ yield following the general procedure $\mathrm{F}$. Reaction time: 11 h. ${ }^{1} \mathrm{H} \mathrm{NMR}\left(400 \mathrm{MHz}, \mathrm{CDCl}_{3}\right)$ d: $5.92(\mathrm{~d}, 1 \mathrm{H}, \mathrm{J}=15.6 \mathrm{~Hz}), 5.55(\mathrm{dt}, 1 \mathrm{H}, \mathrm{J}=15.6$, $6.8 \mathrm{~Hz}$ ), 5.24 (t, 1H, J = 7.6 Hz), 3.59 (t, $2 \mathrm{H}, \mathrm{J}=6.8 \mathrm{~Hz}$ ), 2.16 (q, $2 \mathrm{H}, \mathrm{J}=7.2 \mathrm{~Hz}$ ), 2.06 (q, $2 \mathrm{H}, \mathrm{J}=7.2 \mathrm{~Hz}), 1.41-1.32(\mathrm{~m}, 11 \mathrm{H}), 0.90-0.86(\mathrm{~m}, 12 \mathrm{H}), 0.03(\mathrm{~s}, 6 \mathrm{H}) ;{ }^{13} \mathrm{C}$ NMR $(100$ $\left.\mathrm{MHz}, \mathrm{CDCl}_{3}\right) \delta: 175.6,146.4,129.6,124.9,115.8,62.0,39.1,34.2,29.5,27.3,25.9$, 22.2, 18.3, 13.6, -5.3; IR (neat): 2959, 2931, 2860, 1753, 1600, 1464, 1258, 1125, 958, 836; $\mathrm{MS}\left(\mathrm{ES}^{+}\right)$Calculated for $\left[\mathrm{C}_{20} \mathrm{H}_{38} \mathrm{O}_{3} \mathrm{SiNa}\right]^{+}$377.3; Found: 377.0 .

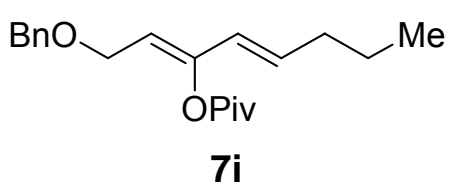

Compound $7 \mathbf{i}$ was isolated in $36 \%$ yield following the general procedure $\mathrm{F}$. Reaction time: 10 h. ${ }^{1} \mathrm{H}$ NMR $\left(400 \mathrm{MHz}, \mathrm{CDCl}_{3}\right)$ ס: 7.35-7.25 (m, 5H), $5.96(\mathrm{~d}, 1 \mathrm{H}, \mathrm{J}=15.6 \mathrm{~Hz}), 5.67$ (dt, $1 \mathrm{H}, \mathrm{J}=15.6,7.2 \mathrm{~Hz}), 5.42(\mathrm{t}, 1 \mathrm{H}, \mathrm{J}=6.8 \mathrm{~Hz}), 4.48(\mathrm{~s}, 2 \mathrm{H}), 3.97(\mathrm{~d}, 2 \mathrm{H}, \mathrm{J}=6.8 \mathrm{~Hz}), 2.08$ (q, $2 \mathrm{H}, \mathrm{J}=6.8 \mathrm{~Hz}), 1.46-1.36(\mathrm{~m}, 2 \mathrm{H}), 1.26(\mathrm{~s}, 9 \mathrm{H}), 0.89(\mathrm{t}, 3 \mathrm{H}, \mathrm{J}=7.6 \mathrm{~Hz}) ;{ }^{13} \mathrm{C} \mathrm{NMR}$ $\left(100 \mathrm{MHz}, \mathrm{CDCl}_{3}\right) \delta: 175.6,147.5,138.1,131.9,128.4,127.8,127.6,124.4,115.5,72.1$, 64.1, 39.1, 34.2, 27.2, 22.1, 13.6; IR (neat): 3033, 2961, 2932, 2873, 1752, 1605, 1455, 1271, 1122, 960; MS (ES ${ }^{+}$) Calculated for $\left[\mathrm{C}_{20} \mathrm{H}_{28} \mathrm{O}_{3} \mathrm{Na}\right]^{+}$339.2; Found: 339.1 .<smiles>CCCCC(=O)/C=C/COCc1ccccc1</smiles>

Compound was isolated in $30 \%$ yield following the general F. ${ }^{1} \mathrm{H} \mathrm{NMR}\left(400 \mathrm{MHz}, \mathrm{CDCl}_{3}\right)$ ס: 7.39-7.28 (m, 5H), $6.83(\mathrm{dt}, 1 \mathrm{H}, \mathrm{J}=16.0,4.4 \mathrm{~Hz}), 6.38(\mathrm{dt}, 1 \mathrm{H}, \mathrm{J}=6.8,2.0 \mathrm{~Hz}), 4.57$ 
(s, 2H), $4.20(\mathrm{dd}, 2 \mathrm{H}, \mathrm{J}=4.4,2.0 \mathrm{~Hz}), 2.55(\mathrm{t}, 2 \mathrm{H}, \mathrm{J}=7.6 \mathrm{~Hz}), 1.64-1.56(\mathrm{~m}, 2 \mathrm{H}), 1.38-$ $1.29(\mathrm{~m}, 2 \mathrm{H}), 0.91(\mathrm{t}, 3 \mathrm{H}, \mathrm{J}=7.6 \mathrm{~Hz}) ;{ }^{13} \mathrm{C} \mathrm{NMR}\left(100 \mathrm{MHz}, \mathrm{CDCl}_{3}\right) \delta: 200.4,141.7,137.6$, 129.3, 128.4, 127.8, 127.6, 72.8, 68.8, 40.3, 26.1, 22.3, 13.8; IR (neat): 3032, 2961, 2933, 2873, 1698, 1677, 1636, 1455, 1362, 1261, 1120, 1100, 1028; MS (ES $\left.{ }^{+}\right)$ Calculated for $\left[\mathrm{C}_{15} \mathrm{H}_{20} \mathrm{O}_{2} \mathrm{Na}\right]^{+}$255.2; Found: 255.2 .

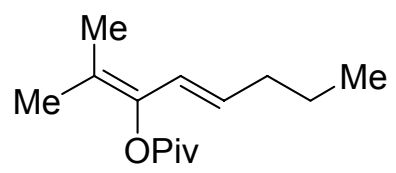

$7 \mathbf{j}$

Compound $7 \mathbf{j}$ was isolated in $36 \%$ yield following the general procedure $\mathrm{F}$. Reaction time: 7 h. ${ }^{1} \mathrm{H}$ NMR $\left(400 \mathrm{MHz}, \mathrm{CDCl}_{3}\right)$ ס: $6.26(\mathrm{~d}, 1 \mathrm{H}, \mathrm{J}=15.2 \mathrm{~Hz}), 6.52(\mathrm{dt}, 1 \mathrm{H}, \mathrm{J}=15.2,6.8$ $\mathrm{Hz}$ ), 2.08 (q, 2H, J = 7.2 Hz), $1.83(\mathrm{~s}, 3 \mathrm{H}), 1.60(\mathrm{~s}, 3 \mathrm{H}), 1.45-1.36(\mathrm{~m}, 2 \mathrm{H}), 1.33(\mathrm{~s}, 9 \mathrm{H})$, $0.91(\mathrm{t}, 3 \mathrm{H}, \mathrm{J}=7.6 \mathrm{~Hz}) ;{ }^{13} \mathrm{C}$ NMR $\left(125 \mathrm{MHz}, \mathrm{CDCl}_{3}\right) \mathrm{\delta}: 176.0,140.1,128.8,121.2,121.0$, 39.1, 34.6, 27.4, 22.5, 18.5, 18.1, 13.6; IR (neat): 2962, 2932, 2874, 1750, 1480, 1460, 1264, 1176, 1127, 955, 889; MS $\left(\mathrm{ES}^{+}\right)$Calculated for $\left[\mathrm{C}_{14} \mathrm{H}_{24} \mathrm{O}_{2} \mathrm{Na}\right]^{+}$247.2; Found: 246.9.

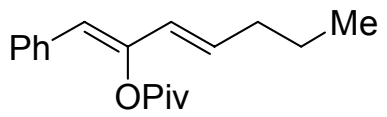

$7 \mathbf{k}$

Compound $\mathbf{7 k}$ was isolated in $11 \%$ yield following the general procedure $\mathrm{F}$. Reaction time: 10 h. ${ }^{1} \mathrm{H}$ NMR $\left(400 \mathrm{MHz}, \mathrm{CDCl}_{3}\right)$ D: 7.40-7.18 (m, 5H), $6.16(\mathrm{~s}, 1 \mathrm{H}), 6.09(\mathrm{~d}, 1 \mathrm{H}, \mathrm{J}=$ $15.6 \mathrm{~Hz}), 5.73(\mathrm{dt}, 1 \mathrm{H}, \mathrm{J}=15.6,6.8 \mathrm{~Hz}), 2.14(\mathrm{q}, 2 \mathrm{H}, \mathrm{J}=6.8 \mathrm{~Hz}), 1.50-1.39(\mathrm{~m}, 2 \mathrm{H})$, $1.34(\mathrm{~s}, 9 \mathrm{H}), 0.93(\mathrm{t}, 3 \mathrm{H}, \mathrm{J}=7.6 \mathrm{~Hz}) ;{ }^{13} \mathrm{C}$ NMR $\left(100 \mathrm{MHz}, \mathrm{CDCl}_{3}\right)$ ठ: 175.3, 146.0, 134.4, 131.4, 128.6, 128.2, 127.2, 125.8, 118.6, 39.1, 34.4, 27.4, 22.3, 13.6; IR (neat): 2960, 2931, 2873, 1752, 1627, 1447, 1269, 1111, 959; MS (ES $\left.{ }^{+}\right)$Calculated for $\left[\mathrm{C}_{18} \mathrm{H}_{24} \mathrm{O}_{2} \mathrm{Na}\right]^{+}$ 295.2; Found: 295.1.

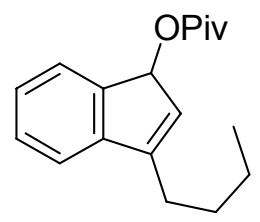

8

Compound 8 was isolated in $69 \%$ yield. ${ }^{1} \mathrm{H}$ NMR $\left(400 \mathrm{MHz}, \mathrm{CDCl}_{3}\right) \delta: 7.37(\mathrm{~d}, 1 \mathrm{H}, \mathrm{J}=7.6$ $\mathrm{Hz}), 7.30(\mathrm{t}, 1 \mathrm{H}, J=7.6 \mathrm{~Hz}), 7.23(\mathrm{~d}, 1 \mathrm{H}, \mathrm{J}=7.2 \mathrm{~Hz}), 7.18(\mathrm{t}, 1 \mathrm{H}, \mathrm{J}=7.6 \mathrm{~Hz}), 6.18(\mathrm{~d}, 1 \mathrm{H}$, 
$J=1.2 \mathrm{~Hz}), 6.03(\mathrm{~d}, 1 \mathrm{H}, \mathrm{J}=1.6 \mathrm{~Hz}), 2.47(\mathrm{t}, 2 \mathrm{H}, \mathrm{J}=7.6 \mathrm{~Hz}), 1.69-1.61(\mathrm{~m}, 2 \mathrm{H}), 1.47-$ $1.38(\mathrm{~m}, 2 \mathrm{H}), 1.23(\mathrm{~s}, 9 \mathrm{H}), 0.95(\mathrm{t}, 3 \mathrm{H}, \mathrm{J}=7.6 \mathrm{~Hz}) ;{ }^{13} \mathrm{C} \mathrm{NMR}\left(100 \mathrm{MHz}, \mathrm{CDCl}_{3}\right)$ ס: 179.0, 147.8, 144.0, 143.1, 128.4, 127.0, 126.0, 123.8, 119.3, 76.5, 38.9, 29.4, 27.2, 27.1, 22.6, 13.9; IR (neat): 2960, 2932, 2874, 1730, 1620, 1480, 1459, 1277, 1151, 1033, 973, 916; MS (ES $\left.{ }^{+}\right)$Calculated for $\left[\mathrm{C}_{18} \mathrm{H}_{24} \mathrm{O}_{2} \mathrm{Na}\right]^{+}$295.2; Found: 295.1.

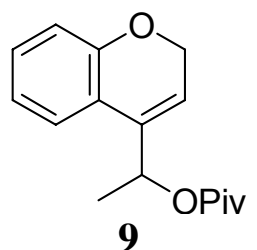

Compound 9 was isolated in $60 \%$ yield following the general procedure $F$. Reaction time: 3 h. ${ }^{1} \mathrm{H}$ NMR $\left(400 \mathrm{MHz}, \mathrm{CDCl}_{3}\right)$ ): $7.20(\mathrm{dd}, 1 \mathrm{H}, \mathrm{J}=7.6,1.6 \mathrm{~Hz}), 7.13(\mathrm{dt}, 1 \mathrm{H}, \mathrm{J}=7.6,1.6$ $\mathrm{Hz}), 6.90(\mathrm{dt}, 1 \mathrm{H}, J=7.6,1.2 \mathrm{~Hz}), 6.83(\mathrm{dd}, 1 \mathrm{H}, J=8.0,1.2 \mathrm{~Hz}), 5.86-5.80(\mathrm{~m}, 2 \mathrm{H}), 4.76$ $(\mathrm{d}, 2 \mathrm{H}, \mathrm{J}=4.0 \mathrm{~Hz}), 1.45(\mathrm{~d}, 3 \mathrm{H}, \mathrm{J}=6.4 \mathrm{~Hz}), 1.21(\mathrm{~s}, 9 \mathrm{H}) ;{ }^{13} \mathrm{C} \mathrm{NMR}\left(100 \mathrm{MHz}, \mathrm{CDCl}_{3}\right) \delta$ : 177.5, 154.4, 134.7, 129.1, 123.5, 121.2, 121.2, 118.2, 116.3, 68.3, 65.0, 38.7, 27.1, 19.5; IR (neat): 2978, 2873, 1729, 1605, 1481, 1452, 1282, 1223, 1158, 1039; MS (ES $\left.{ }^{+}\right)$ Calculated for $\left[\mathrm{C}_{16} \mathrm{H}_{20} \mathrm{O}_{3} \mathrm{Na}\right]^{+}$283.2; Found: 283.0.

\section{One-pot, tandem diene formation and Diels-Alder reaction}
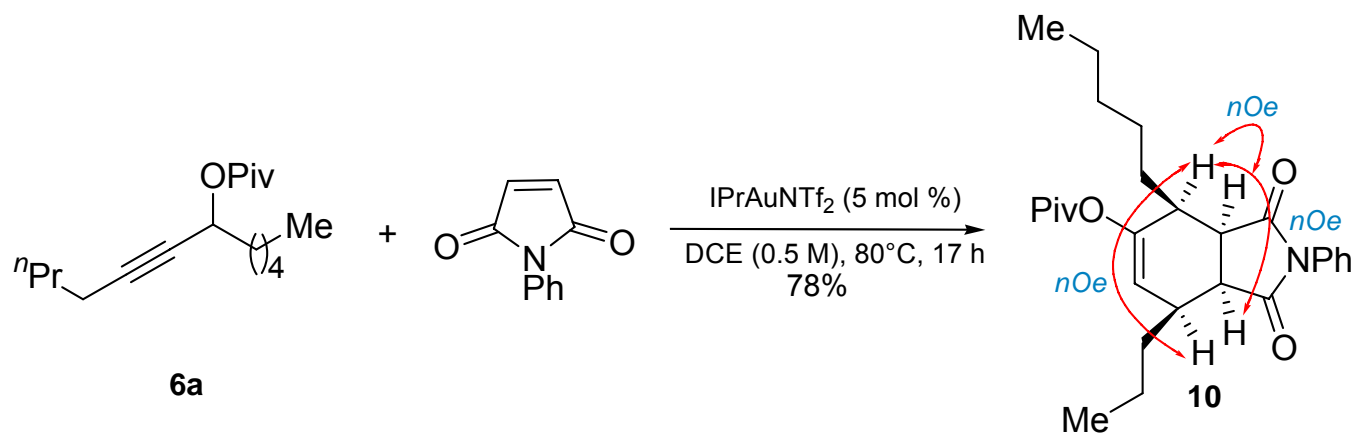

To a $0.5 \mathrm{M}$ solution of propargyl pivaloate $6 \mathbf{a}(0.272 \mathrm{~g}, 1.023 \mathrm{mmol})$ and $\mathrm{N}$ phenylphthalimide $(0.354 \mathrm{~g}, 2.05 \mathrm{mmol})$ in anhydrous 1,2-dichloroethane $(2 \mathrm{~mL})$ in a flame-dried 2-dram via was added $5 \mathrm{~mol} \%$ of $\operatorname{IPrAuNTf}_{2}(44 \mathrm{mg}, 0.05 \mathrm{mmol}$ ). The vial was sealed tightly with Teflon tape inside and outside the cap and heated up to $80^{\circ} \mathrm{C}$ for $17 \mathrm{~h}$. The reaction mixture was concentrated under vacuum, and the resulting residue was purified via silica gel flash column chromatography (eluents: EtOAc : hexanes =1:5) to yield $0.352 \mathrm{~g}$ of pure cycloadduct 10 (78\% yield). ${ }^{1} \mathrm{H}$ NMR $\left(500 \mathrm{MHz}, \mathrm{CDCl}_{3}\right)$ ס: $7.44-$ 
$7.40(\mathrm{~m}, 2 \mathrm{H}), 7.35-7.31(\mathrm{~m}, 3 \mathrm{H}), 5.38(\mathrm{t}, 1 \mathrm{H}, \mathrm{J}=3.5 \mathrm{~Hz}), 3.34(\mathrm{dd}, 1 \mathrm{H}, \mathrm{J}=6.8,4.4 \mathrm{~Hz}$ ), $3.26(\mathrm{dd}, 1 \mathrm{H}, \mathrm{J}=7.6 \mathrm{~Hz}), 2.61-2.59(\mathrm{~m}, 1 \mathrm{H}), 2.38-2.35(\mathrm{~m}, 1 \mathrm{H}), 2.05-1.82(\mathrm{~m}, 3 \mathrm{H}), 1.73-$ $1.60(\mathrm{~m}, 2 \mathrm{H}), 1.56-1.27(\mathrm{~m}, 8 \mathrm{H}), 1.25(\mathrm{~s}, 9 \mathrm{H}), 0.99(\mathrm{t}, 3 \mathrm{H}, \mathrm{J}=7.5 \mathrm{~Hz}), 0.90(\mathrm{t}, 3 \mathrm{H}, \mathrm{J}=7.0$ $\mathrm{Hz}) ;{ }^{13} \mathrm{C}$ NMR $\left(125 \mathrm{MHz}, \mathrm{CDCl}_{3}\right) \delta: 176.5,176.4,175.7,149.1,132.2,128.8,128.3$, 126.9, 117.8, 44.1, 43.4, 39.2, 38.9, 36.7, 33.4, 31.9, 27.7, 27.1, 25.9, 22.6, 21.3, 14.0, 14.0; IR (neat): 2958, 2930, 2872, 1750, 1711, 1600, 1456, 1384, 1119; $M S\left(\mathrm{ES}^{+}\right)$ Calculated for $\left[\mathrm{C}_{27} \mathrm{H}_{37} \mathrm{NO}_{4} \mathrm{Na}\right]^{+}$462.3; Found: 462.3 .

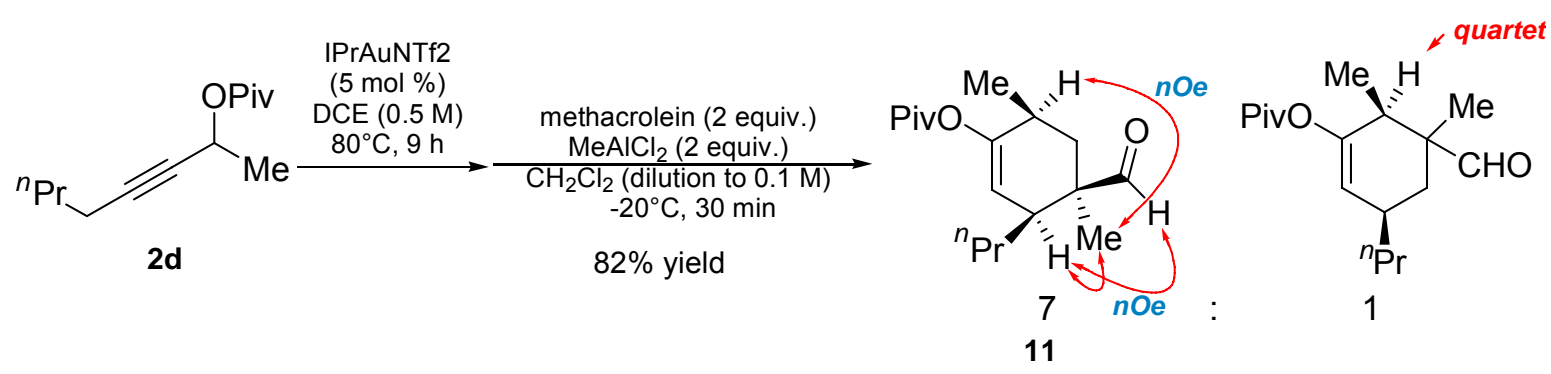

To a solution of propargyl pivaloate $2 \mathrm{~d}(0.249 \mathrm{~g}, 1.19 \mathrm{mmol})$ in anhydrous 1,2dichloroethane $(2.4 \mathrm{~mL})$ in a flame-dried 2-dram via was added IPrAuNTf $\mathrm{I}_{2}(51 \mathrm{mg}, 0.06$ $\mathrm{mmol})$. The vial was sealed tightly with Teflon tape inside and outside the cap and heated up to $80^{\circ} \mathrm{C}$ for $9 \mathrm{~h}$. The reaction mixture was transferred to a $50-\mathrm{mL}$ round-bottom flask and $\mathrm{CH}_{2} \mathrm{Cl}_{2}(10 \mathrm{~mL})$ was added. The resulting mixture was cooled to $-20^{\circ} \mathrm{C}$ and treated with methacrolein $(167 \mathrm{mg}, 2.38 \mathrm{mmol})$ and $\mathrm{MeAICl}_{2}(2.4 \mathrm{~mL}, 1 \mathrm{M}$ solution in hexanes) for $30 \mathrm{~min}$. Then, the reaction was quenched with $\mathrm{Et}_{3} \mathrm{~N}(1 \mathrm{~mL})$ and diluted with saturated aqueous $\mathrm{NH}_{4} \mathrm{Cl}$ solution $(20 \mathrm{~mL})$. Extraction with $\mathrm{Et}_{2} \mathrm{O}(3 \times 20 \mathrm{~mL})$ and the combined organic layers were dried $\left(\mathrm{MgSO}_{4}\right)$, filtered, and concentrated. The resulting residue was purified via silica gel flash column chromatography (eluents: EtOAc : hexanes $=1: 8$ ) to yield an inseparable mixture of cycloadduct 11 and a structural isomer in a combined $82 \%$ yield (ratio: 7:1). Compound 11: ${ }^{1} \mathrm{H}$ NMR $\left(500 \mathrm{MHz}, \mathrm{CDCl}_{3}\right)$ ס: 9.56 (s, $1 \mathrm{H}$, major), $9.52(\mathrm{~s}, 1 \mathrm{H}$, minor), 5.37 (dd, $1 \mathrm{H}, \mathrm{J}=6.0,2.0 \mathrm{~Hz}$, major), $5.23(\mathrm{~s}, 1 \mathrm{H}$, minor), 2.57-2.51 (m, 1H), 2.32-2.25 (m, 1H), 1.71 (ddd, $1 \mathrm{H}, \mathrm{J}=14.0,6.5,1.5 \mathrm{~Hz}$ ), 1.61 (dd, $1 \mathrm{H}$, $\mathrm{J}=14.0,11.0 \mathrm{~Hz}), 1.52-1.35(\mathrm{~m}, 2 \mathrm{H}), 1.32-1.17(\mathrm{~m}, 17 \mathrm{H}), 1.04(\mathrm{~d}, 3 \mathrm{H}, \mathrm{J}=7.5 \mathrm{~Hz}$, major $)$, 0.98 (d, 3H, J = 7.0 Hz, minor), 0.93-0.87 (m, 3H); ${ }^{13} \mathrm{C} \mathrm{NMR} \mathrm{(125} \mathrm{MHz,} \mathrm{CDCl}_{3}$ ) ס: 205.5, 177.3, 151.1, 115.1, 48.3, 40.6, 35.9, 32.7, 28.4, 27.2, 27.1, 20.7, 19.6, 17.6, 14.1; IR (neat): 2961, 2934, 2874, 1742, 1733, 1683, 1481, 1461, 1279, 1136, 906; MS (ES $\left.{ }^{+}\right)$ Calculated for $\left[\mathrm{C}_{17} \mathrm{H}_{28} \mathrm{O}_{3} \mathrm{Na}\right]^{+}$303.2; Found: 303.20; For the minor component, it is 
assigned as the regioisomer based on the quartet coupling pattern of the tertiary $\mathrm{H}$ as indicated above.

\section{Preparation of 12}<smiles>COC(=O)/C=C/C(=O)OCCCC#CC(C)OCCCCC(=O)O</smiles>

To a solution of the alcohol $(2.35 \mathrm{mmol})$ and methyl hydrogen fumarate $(2.8 \mathrm{mmol}, 1.1$ eq) in anhydrous $\mathrm{CH}_{2} \mathrm{Cl}_{2}(10 \mathrm{~mL})$ was added catalytic amount DMAP. The solution was cooled in an ice-water bath and treated with EDCI \{1-[3-(dimethylamino)propyl]-3ethylcarbodiimide methiodide $(2.8 \mathrm{mmol}, 1.1 \mathrm{eq})$. The ice-water bath was removed, and the reaction was stirred at room temperature for $0.5 \mathrm{~h}$. The reaction mixture was then diluted with $\mathrm{CH}_{2} \mathrm{Cl}_{2}(20 \mathrm{~mL})$ and washed with water $(3 \times 20 \mathrm{~mL})$ and brine $(20 \mathrm{~mL})$. The combined organic layers were dried (anhydrous $\mathrm{MgSO}_{4}$ ), filtered, and concentrated. The residue was purified through silica gel flash column chromatography (eluents: EtOAc and hexanes $=3: 1)$ to yield ester $12\left(1.5 \mathrm{mmol}\right.$, yield 65\%). ${ }^{1} \mathrm{H} \mathrm{NMR}\left(500 \mathrm{MHz}, \mathrm{CDCl}_{3}\right) \delta$ : $6.86(\mathrm{~s}, 2 \mathrm{H}), 5.40(\mathrm{tq}, 1 \mathrm{H}, \mathrm{J}=6.5,2.0 \mathrm{~Hz}), 4.28(\mathrm{t}, 2 \mathrm{H}, \mathrm{J}=7.0 \mathrm{~Hz}), 3.82(\mathrm{~s}, 3 \mathrm{H}), 2.33(\mathrm{dt}$, $2 \mathrm{H}, \mathrm{J}=7.0,2.0 \mathrm{~Hz}$ ), 2.89 (tt, $2 \mathrm{H}, \mathrm{J}=6.5,6.5 \mathrm{~Hz}), 1.43$ (d, 3H, J = 7.0 Hz), $1.20(\mathrm{~s}, 9 \mathrm{H})$; ${ }^{13} \mathrm{C}$ NMR $\left(100 \mathrm{MHz}, \mathrm{CDCl}_{3}\right)$ ס: 177.4, 165.4, 164.8, 133.6, 133.3, 83.1, 79.9, 63.9, 60.3, 52.3, 38.6, 27.4, 27.0, 21.5, 15.4; IR (neat): 2960, 1728, 1437, 1300, 1153, 1031; MS $\left(\mathrm{ES}^{+}\right)$Calculated for $[\mathrm{C} 17 \mathrm{H} 24 \mathrm{NaO} 6]^{+} 347.2$; Found: 347.1

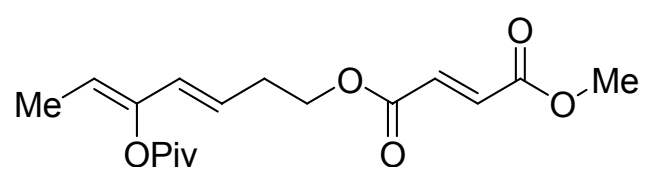

13

Compound 13 was isolated in $67 \%$ yield following the general procedure $\mathrm{F}$. Reaction time: 11 h. ${ }^{1} \mathrm{H}$ NMR (500MHz, $\left.\mathrm{CDCl}_{3}\right) \delta: 6.85(\mathrm{~s}, 2 \mathrm{H}), 6.03(\mathrm{~d}, 1 \mathrm{H}, \mathrm{J}=15.5), 5.61(\mathrm{tt}, 1 \mathrm{H}$, $J=15.5,7 \mathrm{~Hz}), 5.37(\mathrm{q}, 1 \mathrm{H}, \mathrm{J}=7 \mathrm{~Hz}), 4.23(\mathrm{t}, 2 \mathrm{H}, \mathrm{J}=6.5 \mathrm{~Hz}), 3.81(\mathrm{~s}, 3 \mathrm{H}), 2.47(\mathrm{q}, 1 \mathrm{H}$, $\mathrm{J}=6 \mathrm{~Hz}), 1.56(\mathrm{~d}, 3 \mathrm{H}, \mathrm{J}=7 \mathrm{~Hz}), 1.34(\mathrm{~s}, 9 \mathrm{H}) ;{ }^{13} \mathrm{C}$ NMR $\left(100 \mathrm{MHz}, \mathrm{CDCl}_{3}\right) \delta:$ 175.5, 165.4 , 164.8, 145.9, 133.6, 133.4, 127.7, 123.0, 115.8, 64.3, 52.3, 39.2, 31.4, 27.3, 
27.25, 11.1; IR (neat): 2959, 1726, 1603, 1435, 1300, 1126; $M S\left(E S^{+}\right)$Calculated for [C17H24NaO6] $]^{+} 347.2$; Found: 347.0 .

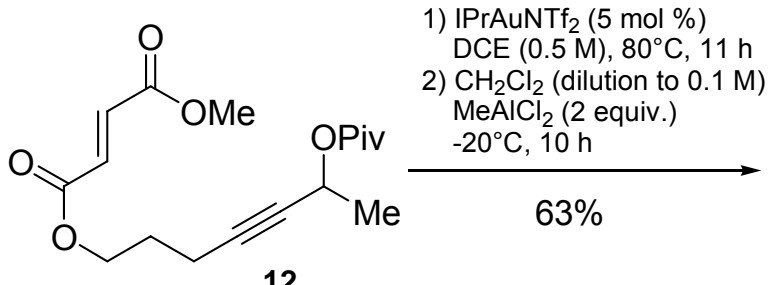

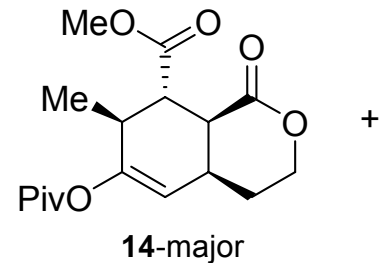

12

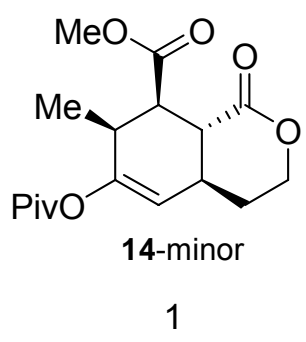

Compound $12(0.178 \mathrm{~g}, 0.55 \mathrm{mmol})$ was subject to the general procedure $\mathrm{F}$. 11 hours later, the reaction mixture was transferred to a $25 \mathrm{~mL}$ round-bottom flask and diluted with $\mathrm{CH}_{2} \mathrm{Cl}_{2}(4 \mathrm{~mL})$. The resulting solution was cooled to $-20^{\circ} \mathrm{C}$ and treated with $\mathrm{MeAlCl}_{2}(1.1$ $\mathrm{mL}, 1 \mathrm{M}$ in hexanes). After stirring at the same temperature for $10 \mathrm{~h}$, the reaction was complete. Then, the reaction was quenched with $\mathrm{Et}_{3} \mathrm{~N}(1 \mathrm{~mL})$ and diluted with saturated aqueous $\mathrm{NH}_{4} \mathrm{Cl}$ solution $(20 \mathrm{~mL})$. Extraction with $\mathrm{Et}_{2} \mathrm{O}(3 \times 20 \mathrm{~mL})$ and the combined organic layers were dried $\left(\mathrm{MgSO}_{4}\right)$, filtered, and concentrated. The resulting residue was purified via silica gel flash column chromatography (eluents: EtOAc : hexanes $=2: 1$ ) to yield cycloadduct $14(0.112 \mathrm{~g}, 63 \%$ yield $)$ as a mixture of diastereomers $(\mathrm{dr}=12: 1)$.

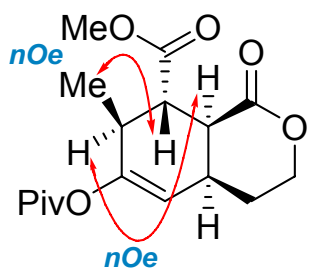

Note: nOe determined by using DMSO- $d_{6}$ as solvent

14-major

${ }^{1} \mathrm{H}$ NMR (400MHz, $\left.\mathrm{CDCl}_{3}\right)$ ס: 5.21-5.19 (m, 1H), 4.55-4.49 (m, 1H), 4.36-4.31 (m, 1H), 5.21-5.19 (m, $1 \mathrm{H}), 3.72(\mathrm{~s}, 3 \mathrm{H}), 3.30-3.25(\mathrm{~m}, 2 \mathrm{H}), 3.03-2.97(\mathrm{~m}, 2 \mathrm{H}), 2.15-2.09(\mathrm{~m}, 1 \mathrm{H})$, 1.83-1.76 (m, 1H), $1.26(\mathrm{~s}, 9 \mathrm{H}), 1.07(\mathrm{~d}, 3 \mathrm{H}, \mathrm{J}=7.2 \mathrm{~Hz}) ;{ }^{13} \mathrm{C}$ NMR (100 MHz, $\left.\mathrm{CDCl}_{3}\right) \delta$ : 176.5, 173.0, 171.2, 151.6, 114.2, 67.2, 52.3, 47.4, 39.5, 38.9, 33.0, 30.0, 27.9, 27.0, 17.6; IR (neat): 2974, 1741, 1458, 1274, 1125; MS (ES ${ }^{+}$) Calculated for [C17H24NaO6] ${ }^{+}$ 347.2; Found: 347.1. 


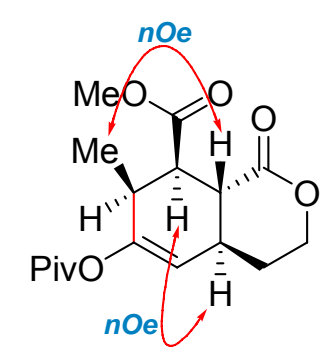

\section{4-minor}

${ }^{1} \mathrm{H}$ NMR (400MHz, $\left.\mathrm{CDCl}_{3}\right)$ ס: $5.34(\mathrm{~d}, 1 \mathrm{H}, \mathrm{J}=2 \mathrm{~Hz}), 4.46-4.20(\mathrm{~m}, 2 \mathrm{H}), 3.75(\mathrm{~s}, 3 \mathrm{H}), 5.61$ (dd, $1 \mathrm{H}, \mathrm{J}=11.6,6 \mathrm{~Hz}), 2.82(\mathrm{t}, 1 \mathrm{H}, \mathrm{J}=12 \mathrm{~Hz}), 2.79-2.74(\mathrm{~m}, 1 \mathrm{H}), 2.64-2.54(\mathrm{~m}, 1 \mathrm{H})$, 2.38-2.28 (m, 1H), 1.77-1.70 (m, 1H), $1.26(\mathrm{~s}, 9 \mathrm{H}), 1.07(\mathrm{~d}, 3 \mathrm{H}, \mathrm{J}=7.2 \mathrm{~Hz}) ;{ }^{13} \mathrm{C} \mathrm{NMR}$ $\left(100 \mathrm{MHz}, \mathrm{CDCl}_{3}\right)$ ס: 176.7, 173.5, 172.3, 150.3, 114.9, 65.2, 51.9, 43.7, 39.0, 37.8, 32.7, 32.2, 27.6, 27.0, 15.2; IR (neat): 2976, 1742, 1603, 1436, 1316, 1274, 1125; MS (ES $\left.{ }^{+}\right)$ Calculated for [C17H24NaO6 $]^{+} 347.2$; Found: 347.0 . 


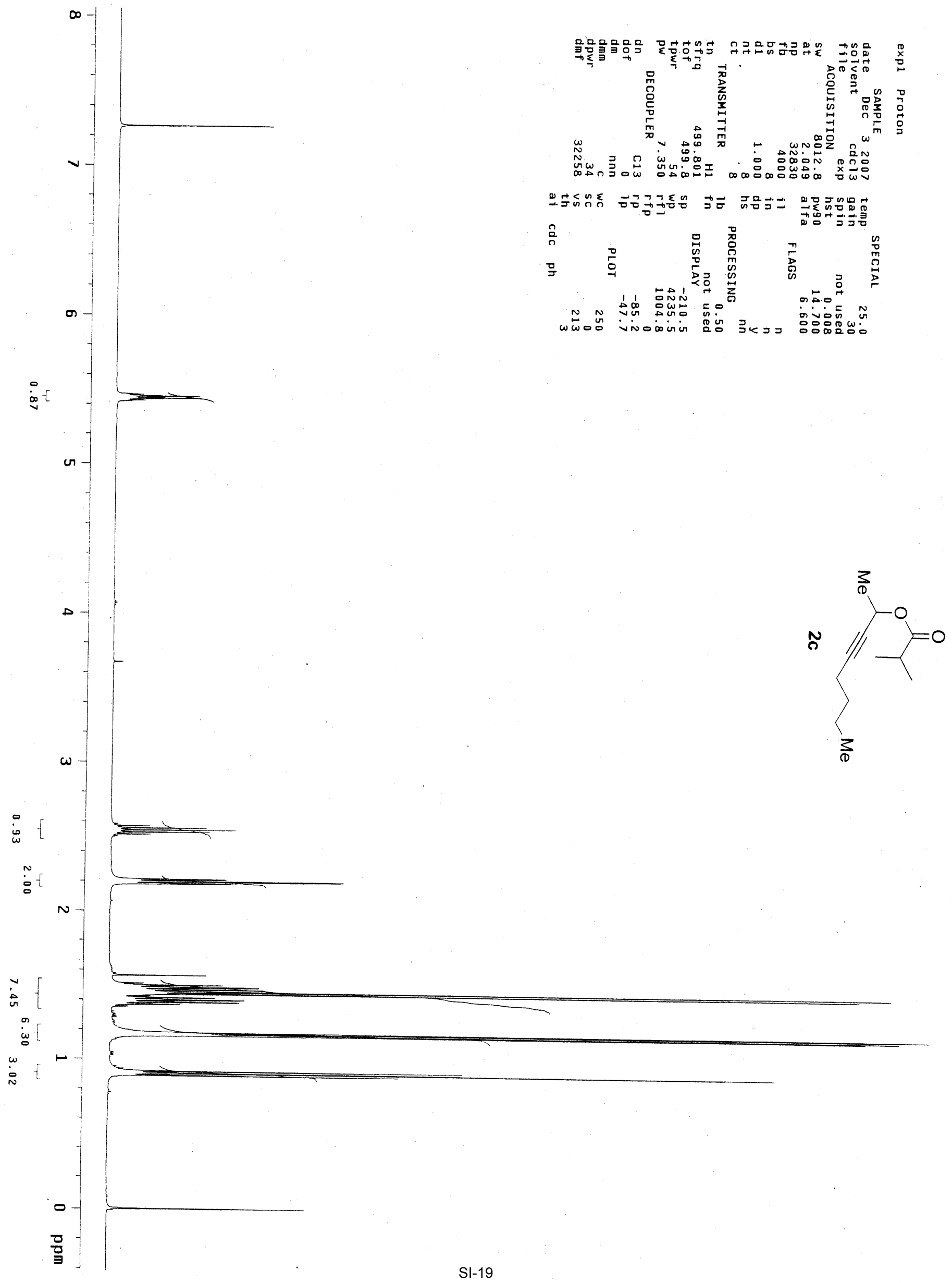




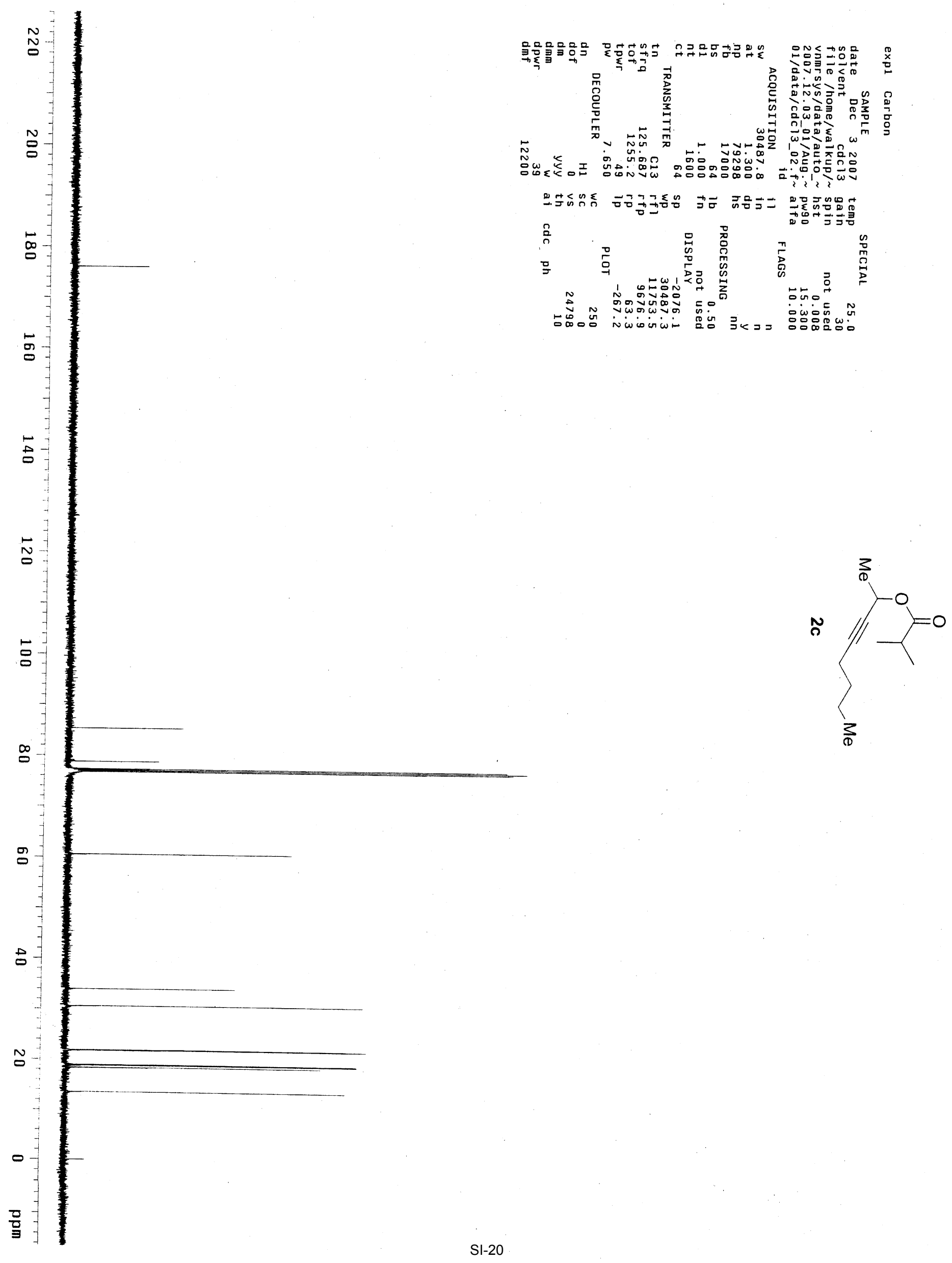




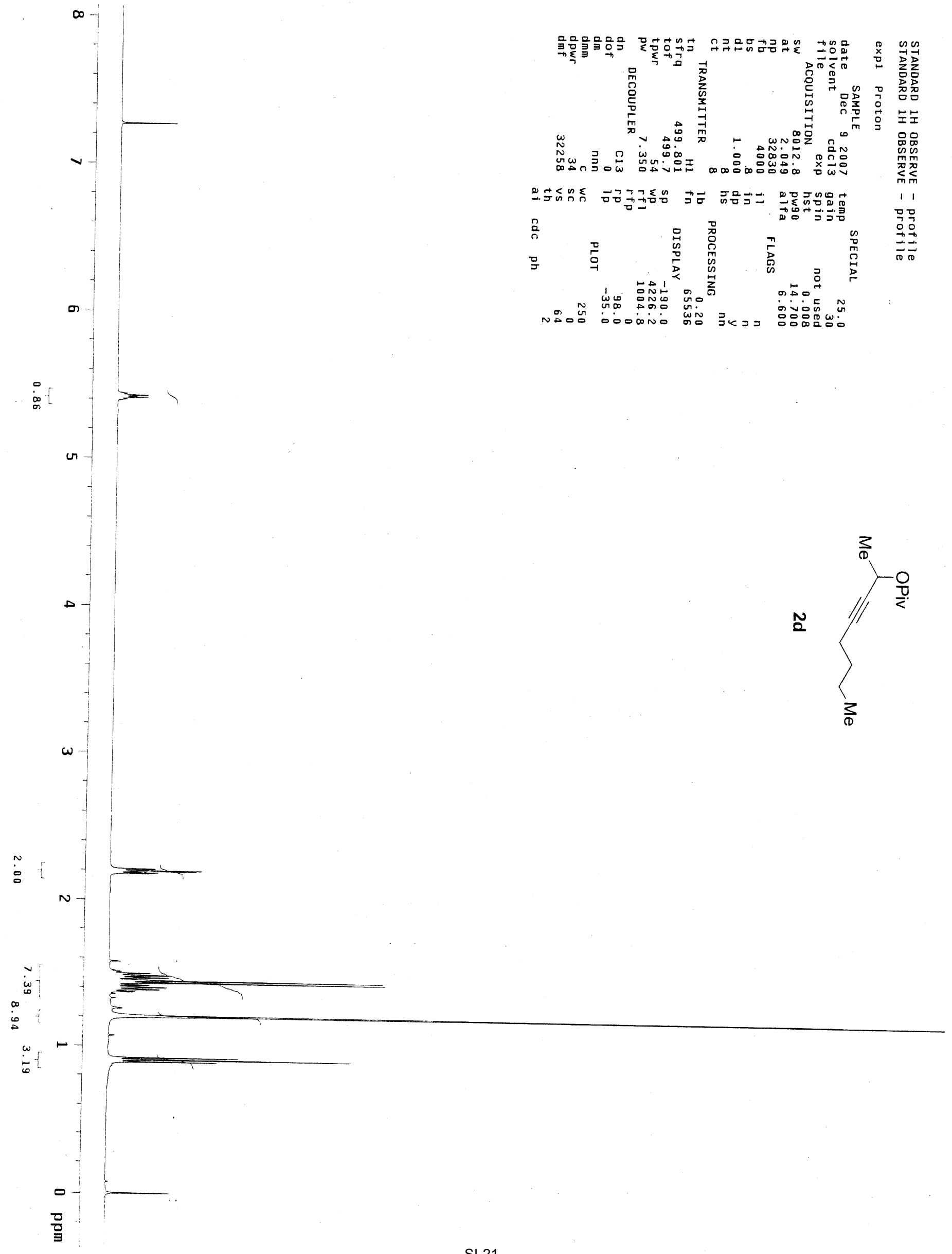




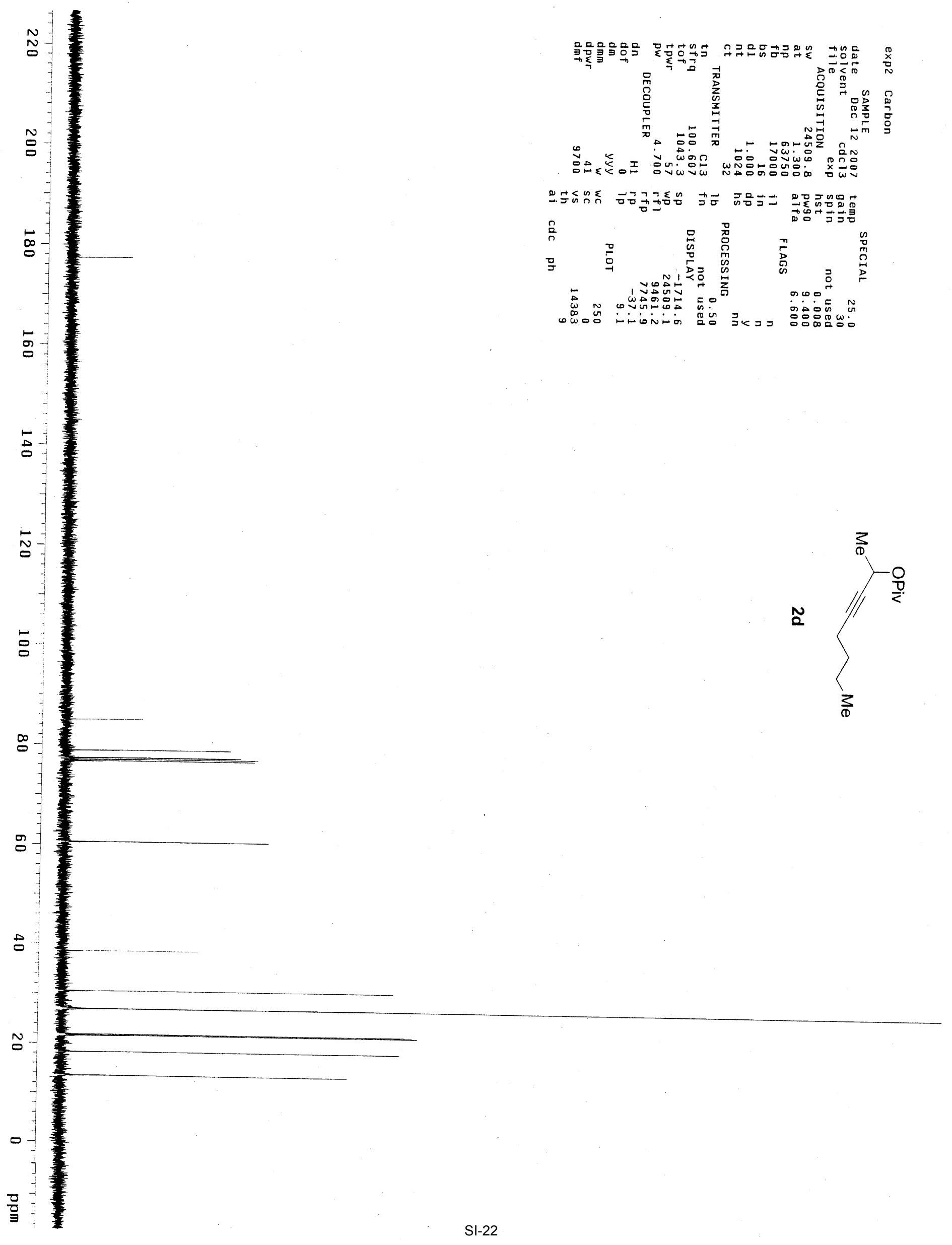




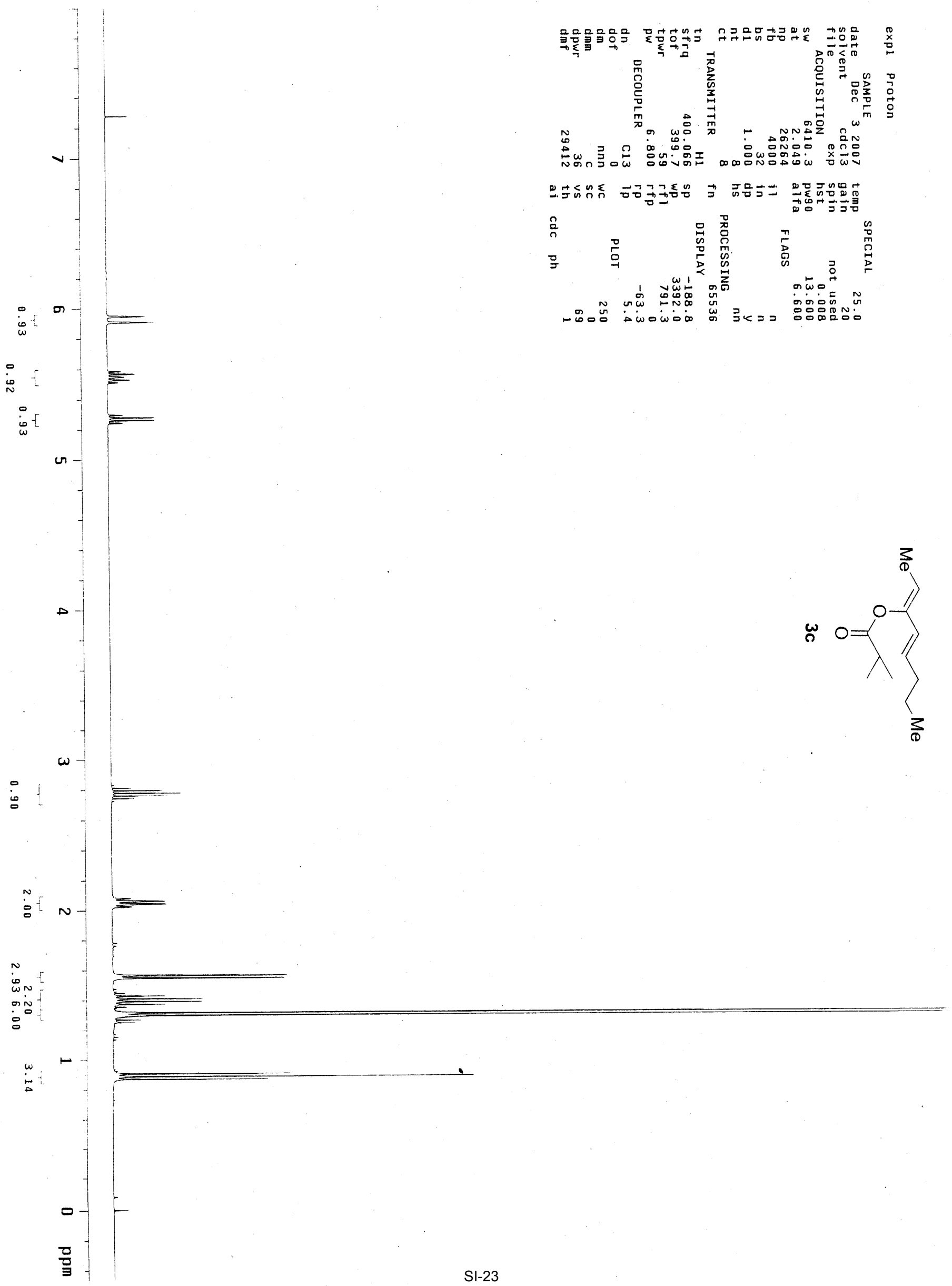




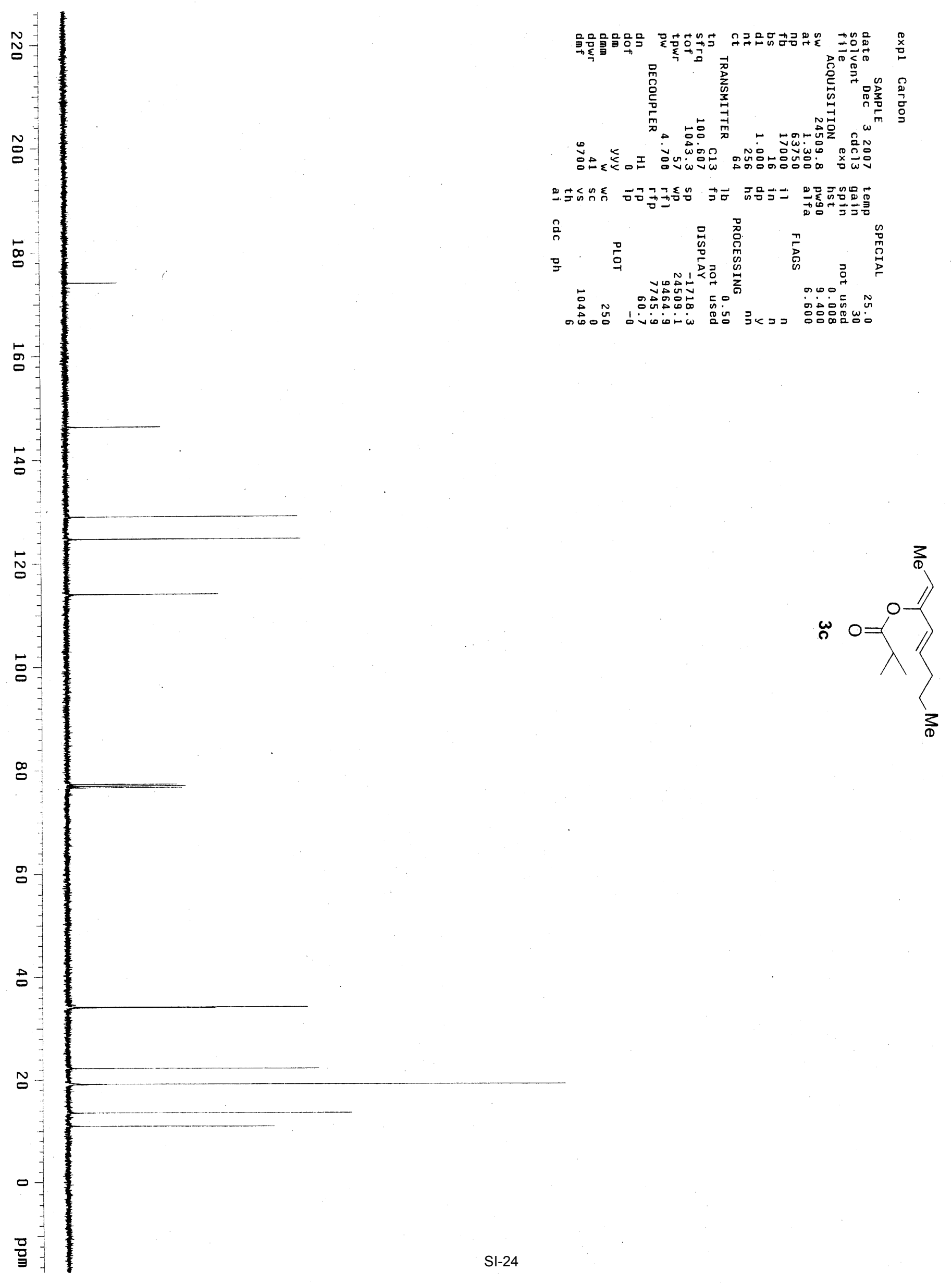




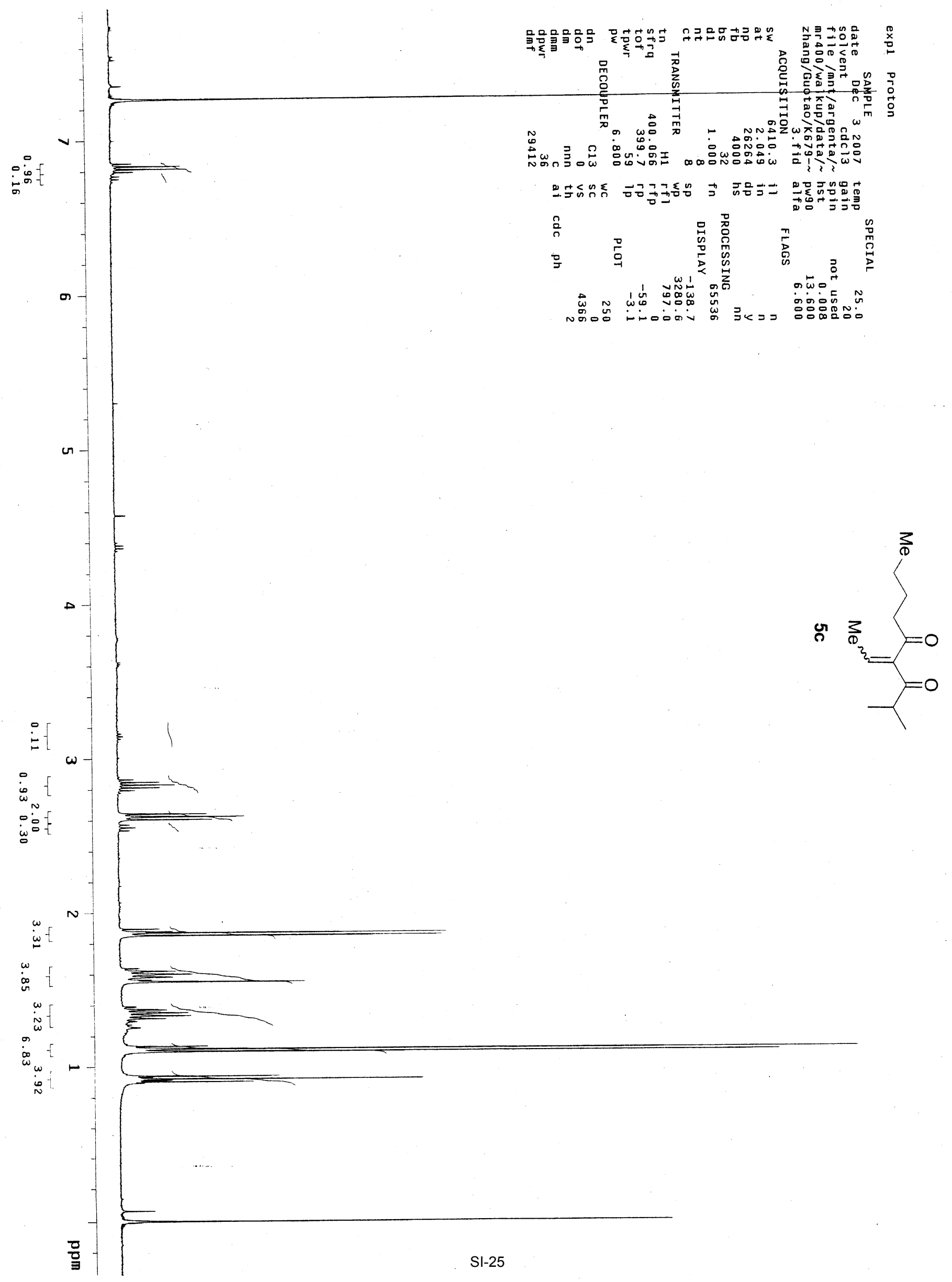




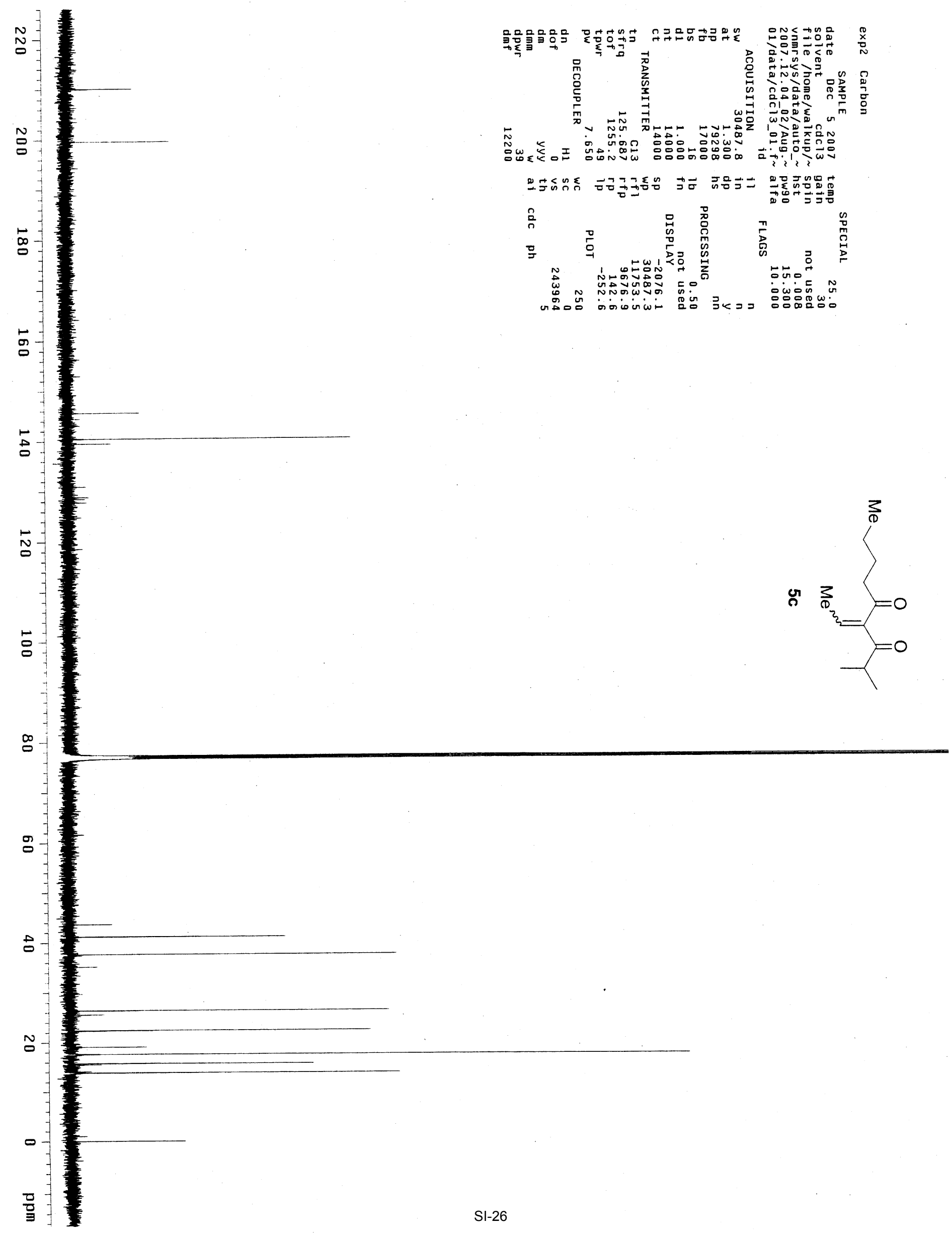




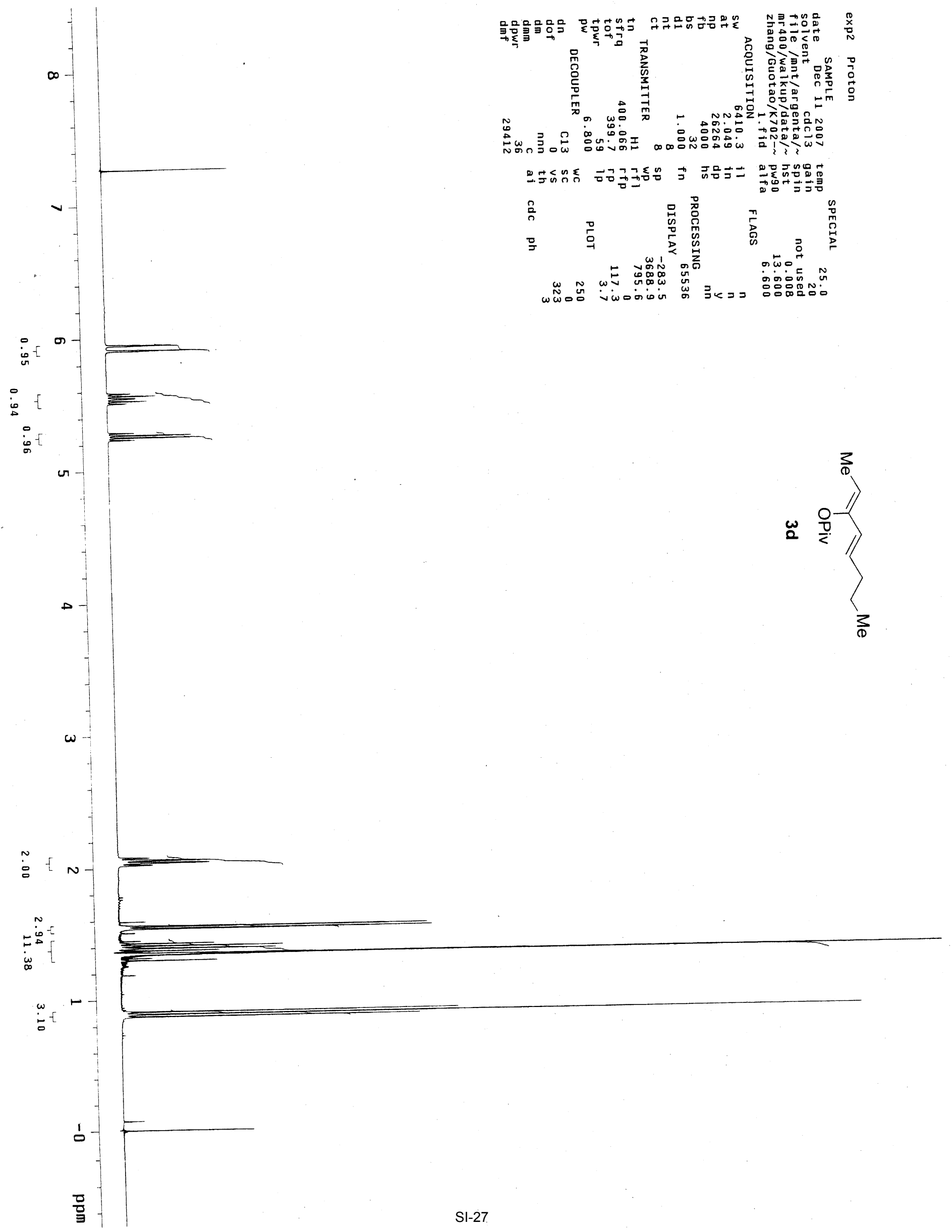




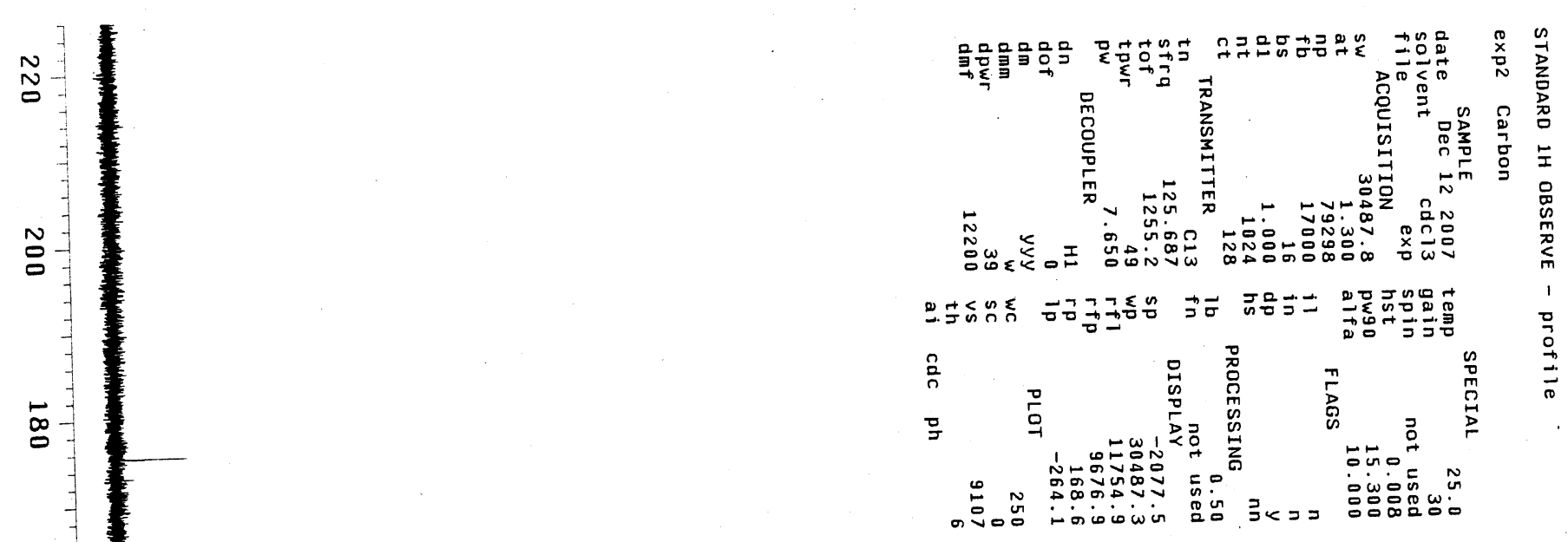

$\underset{\substack{3 \\ 0}}{\substack{3 \\ 0}}$ 


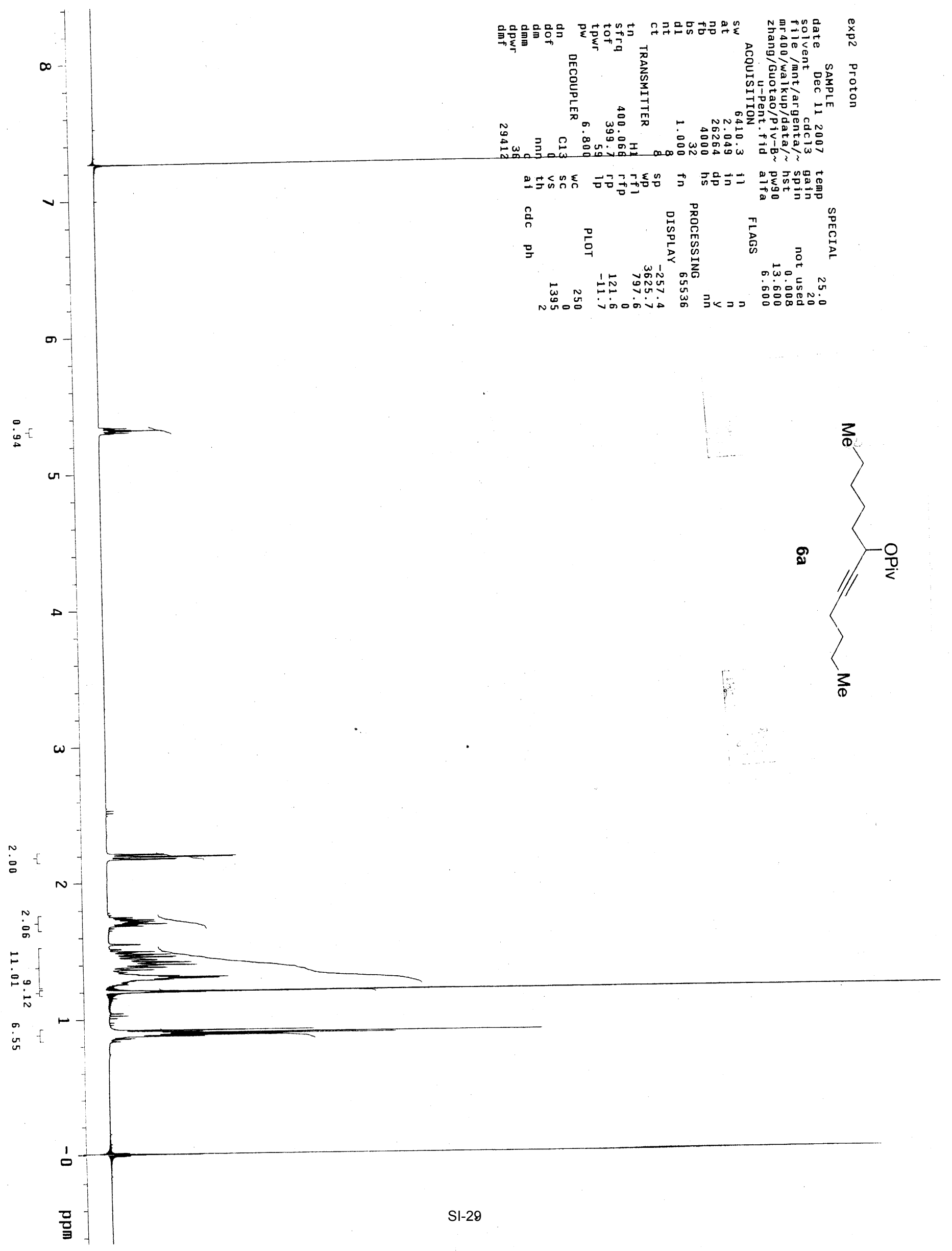



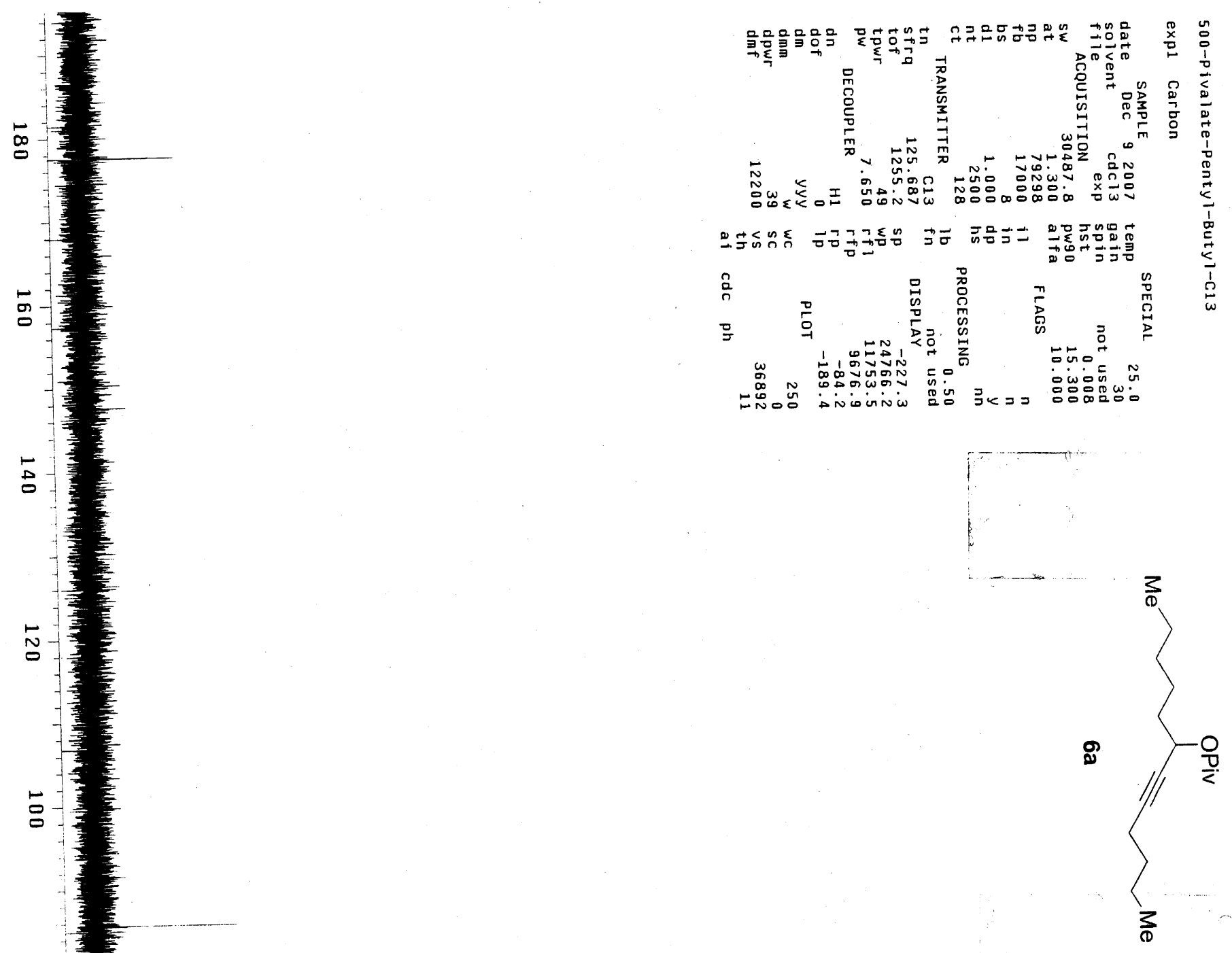


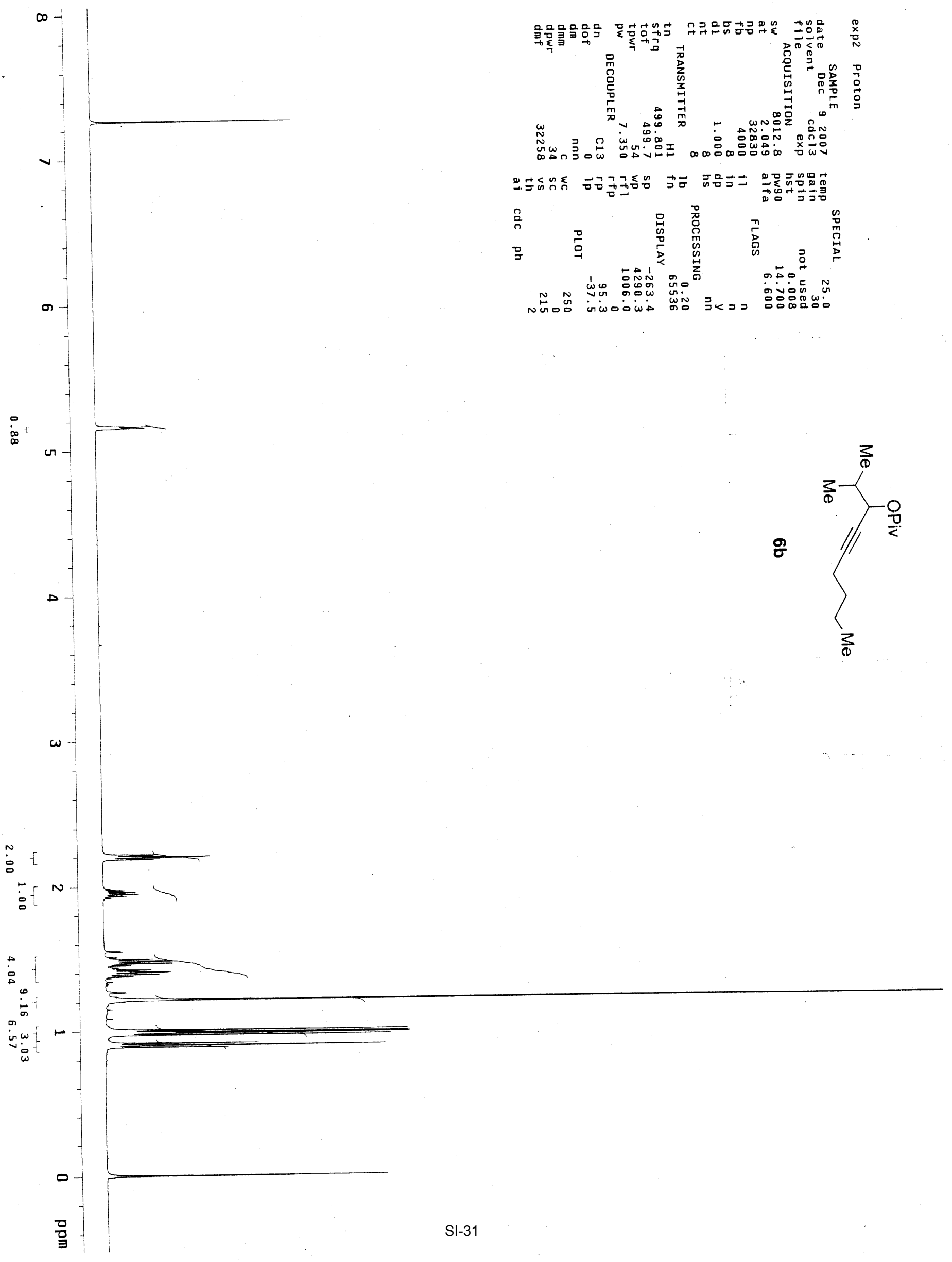




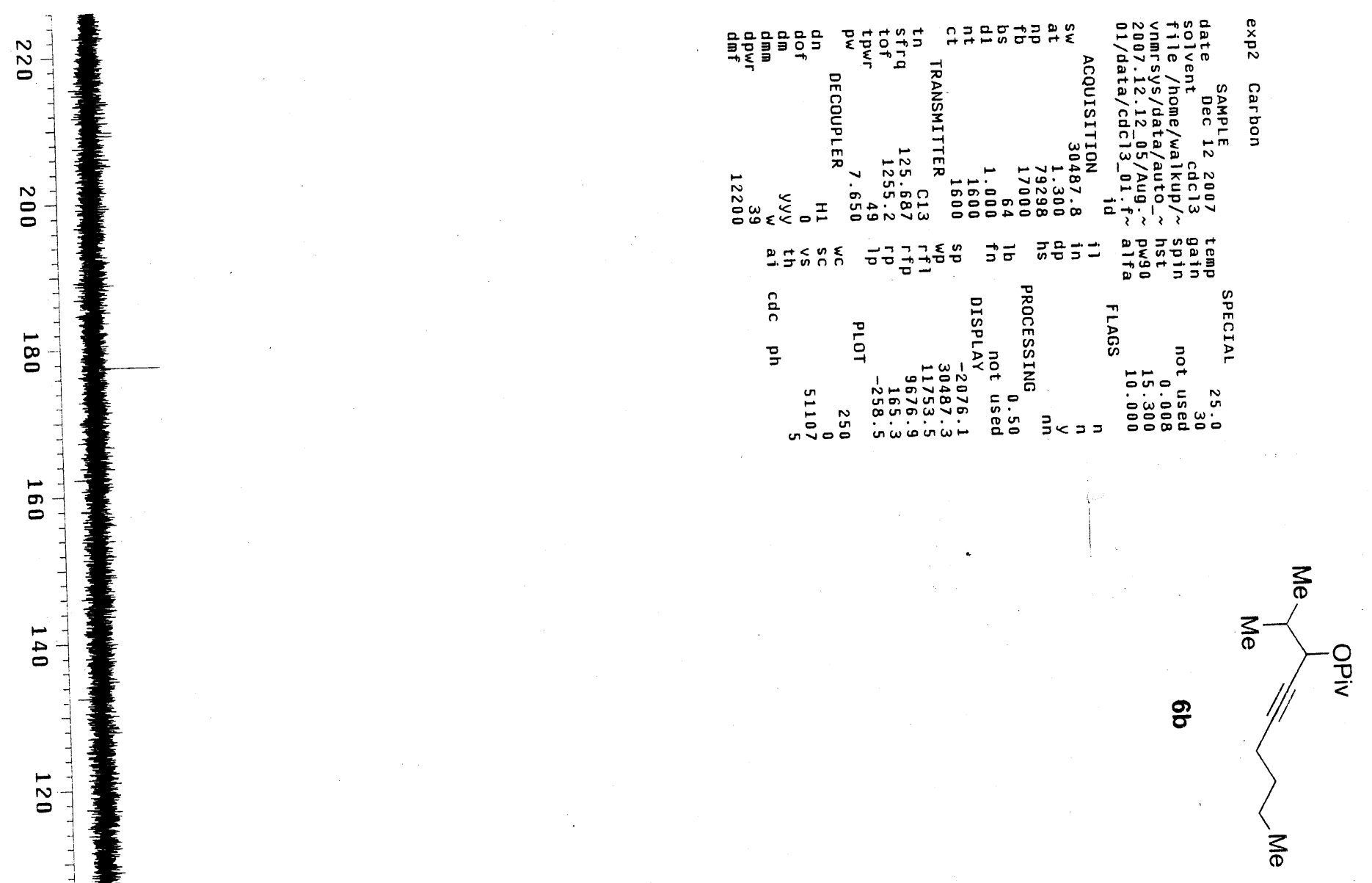




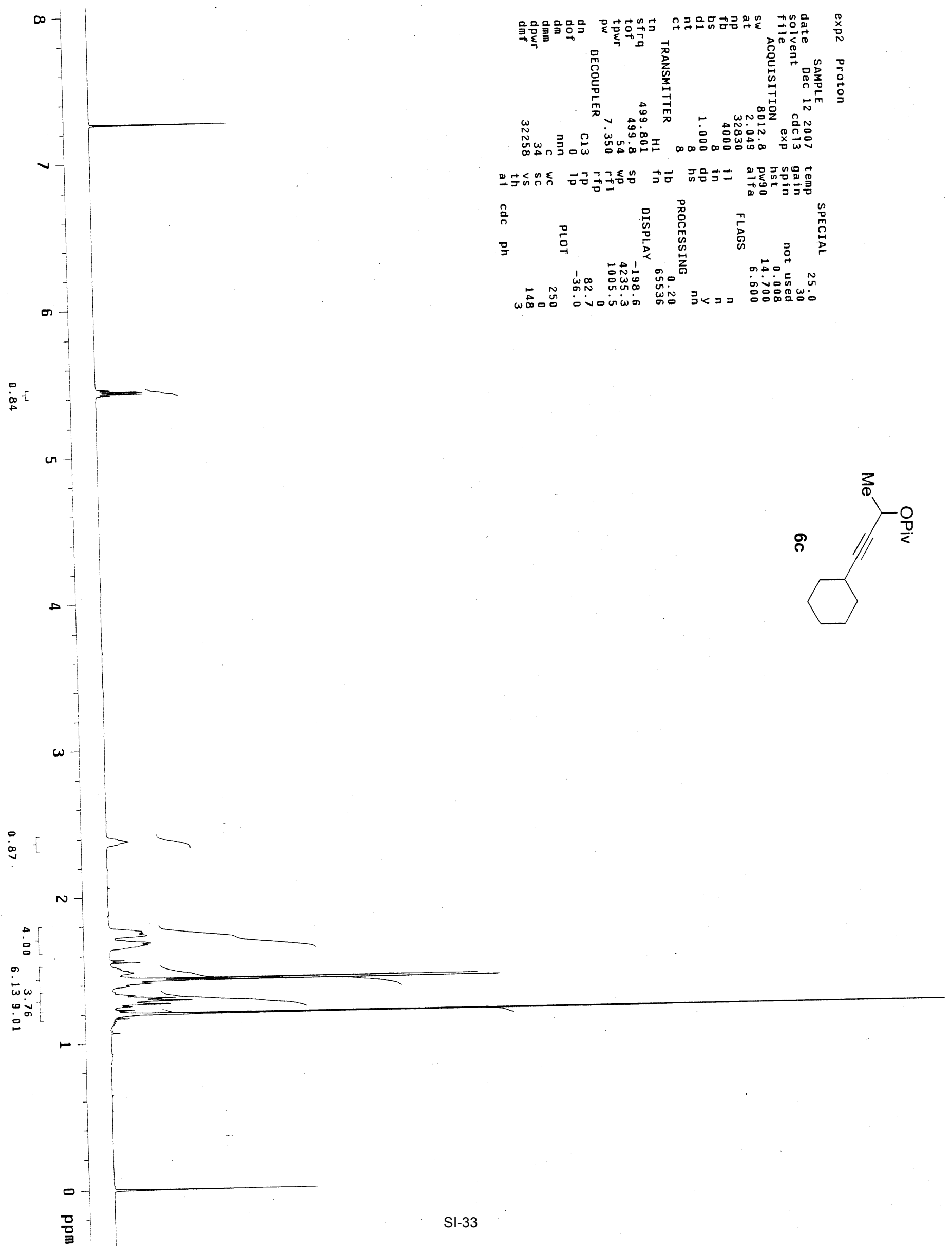




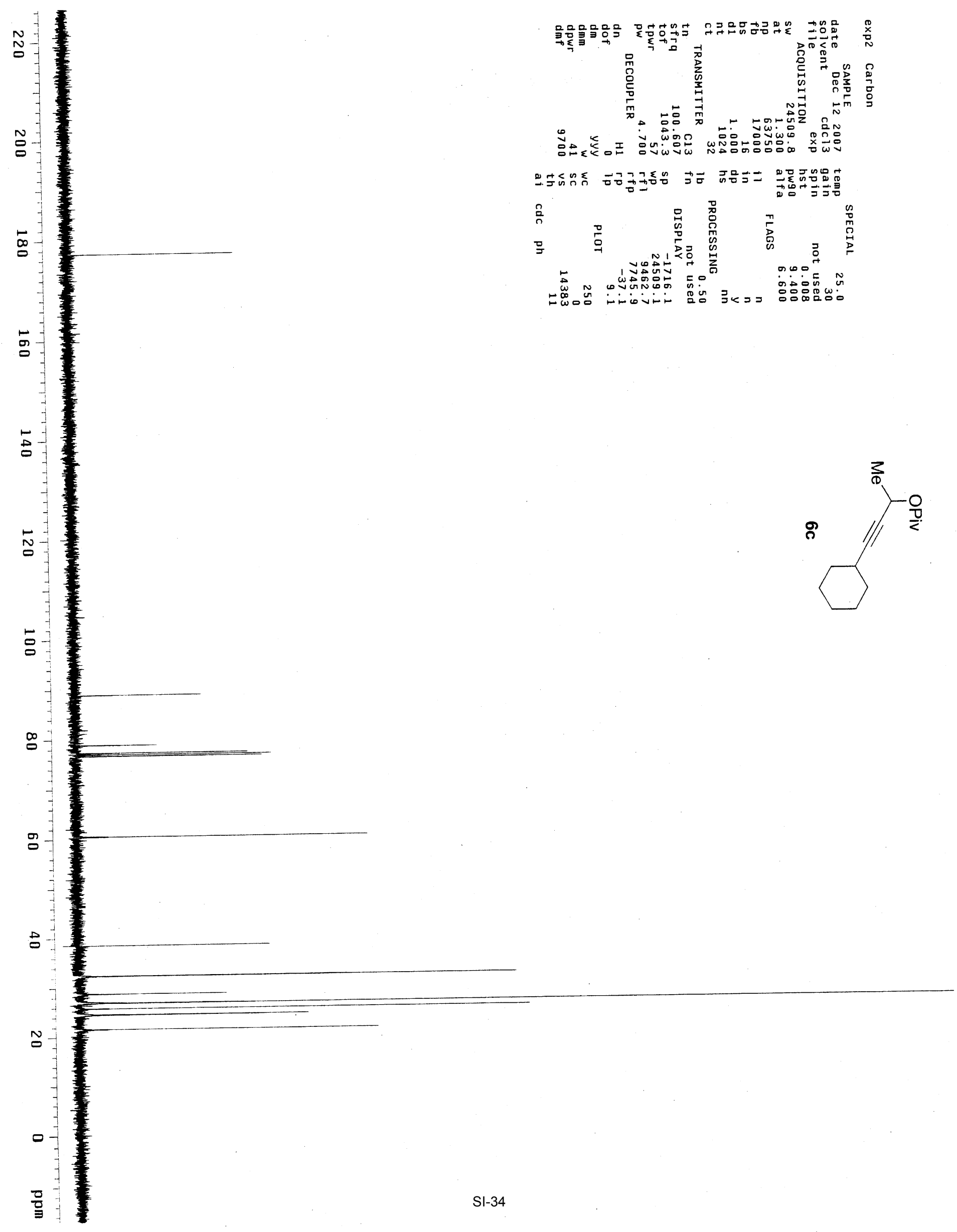




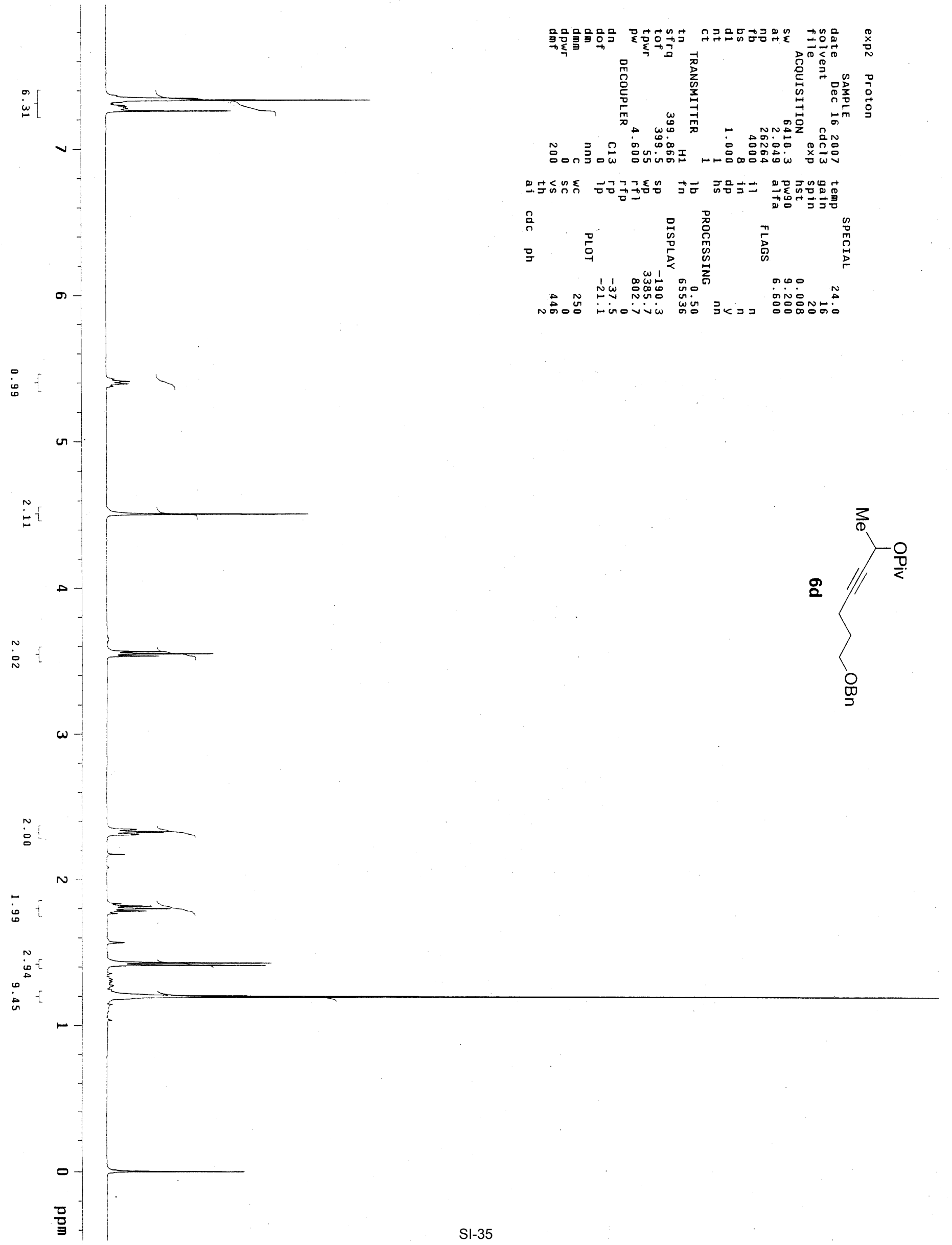




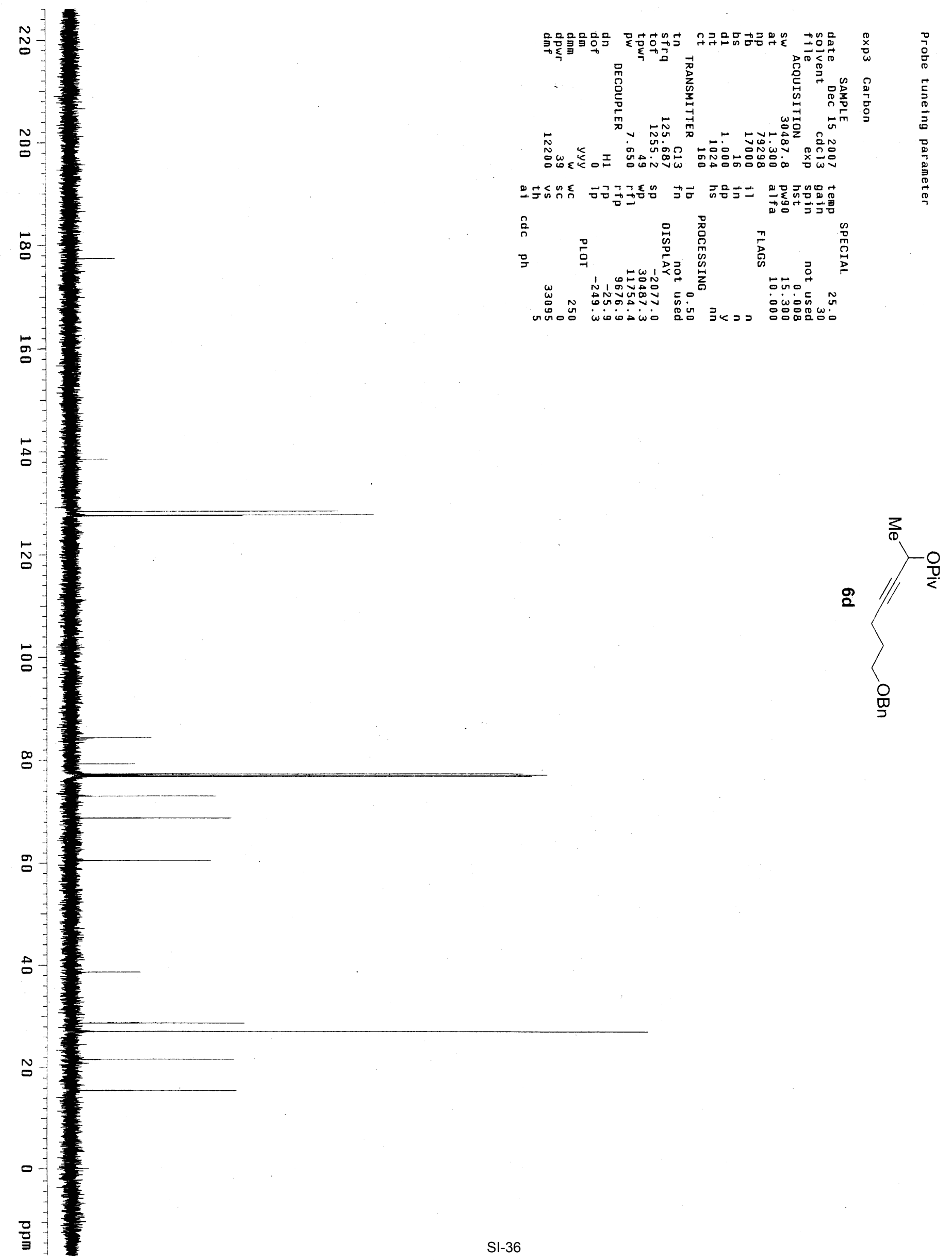




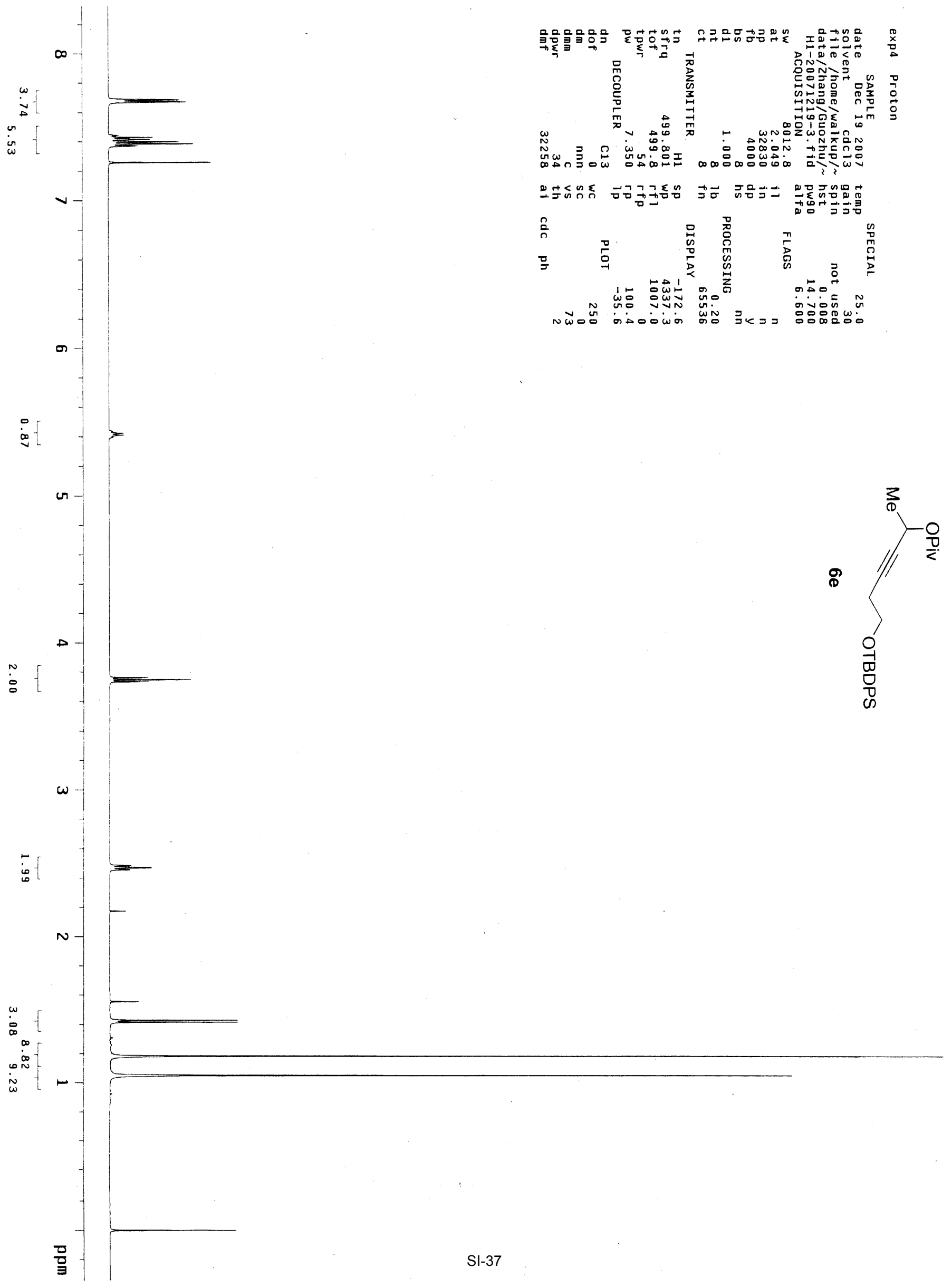




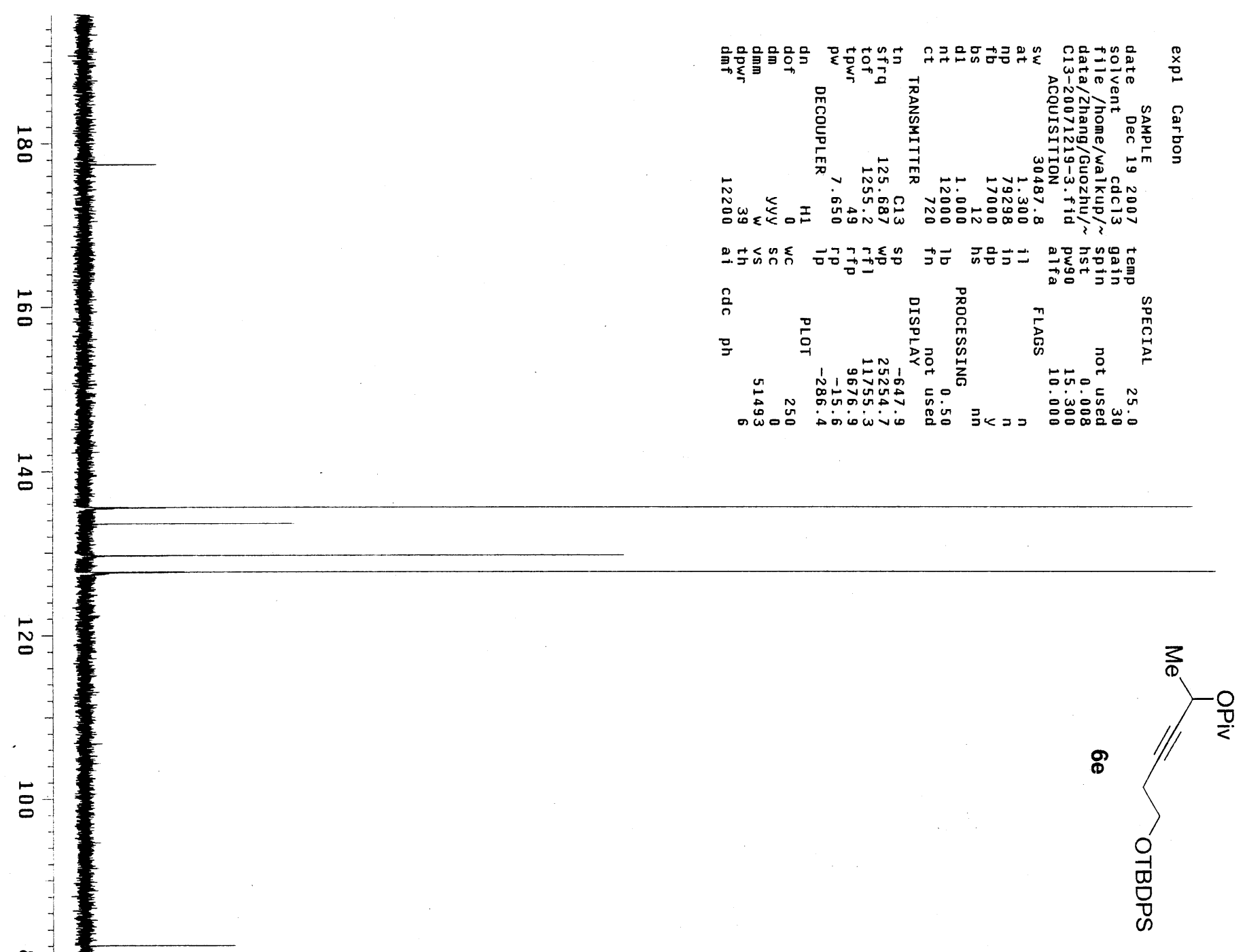




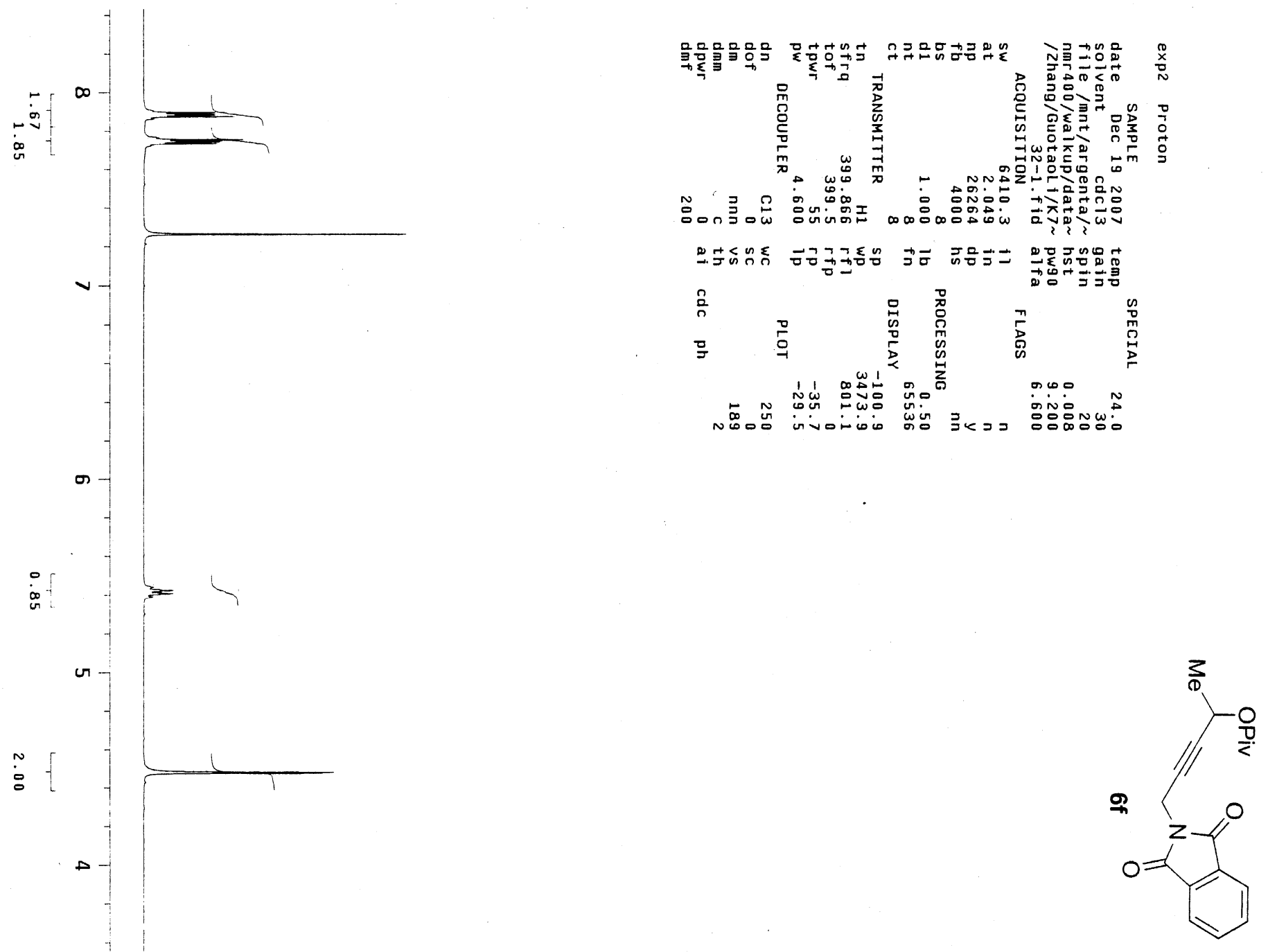

唲 


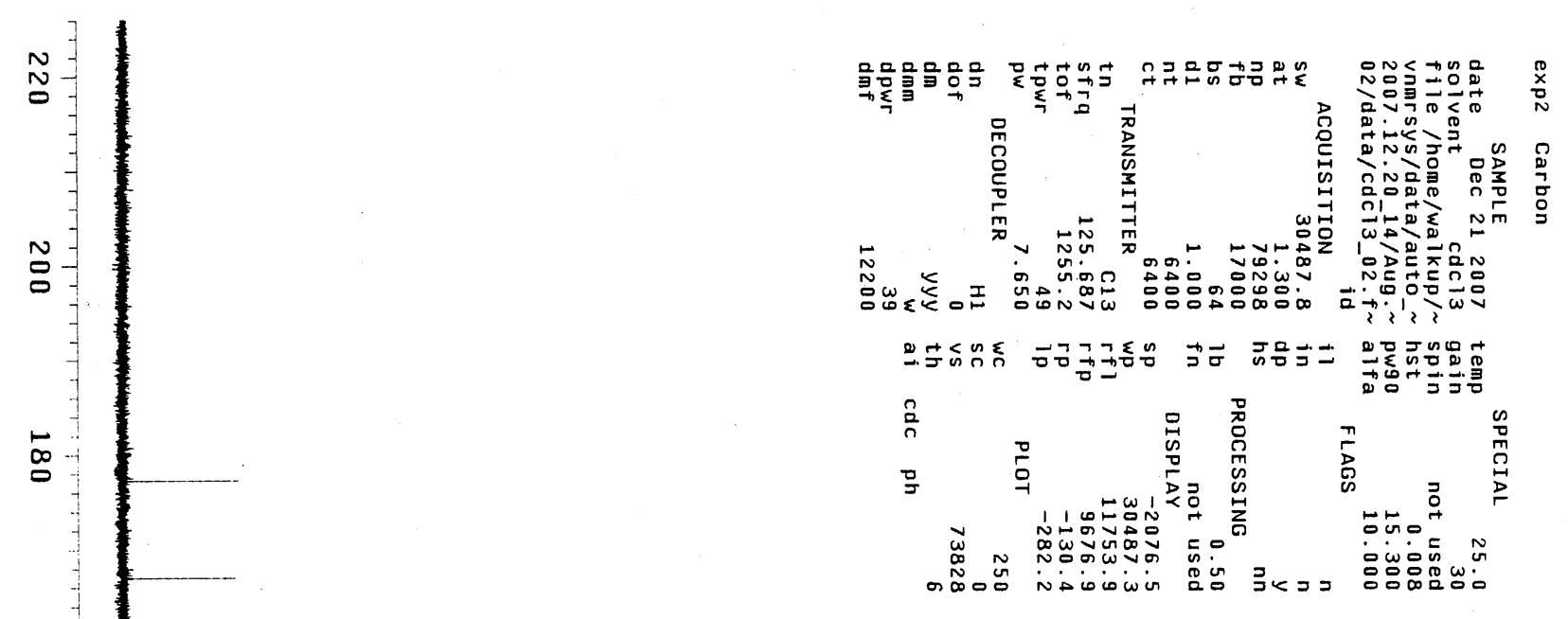

吕

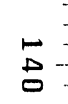

$\stackrel{\Sigma}{\circ}$

宫

$\stackrel{\infty}{\circ}$

8

a

굴

$\triangle$

$-$

을 국 


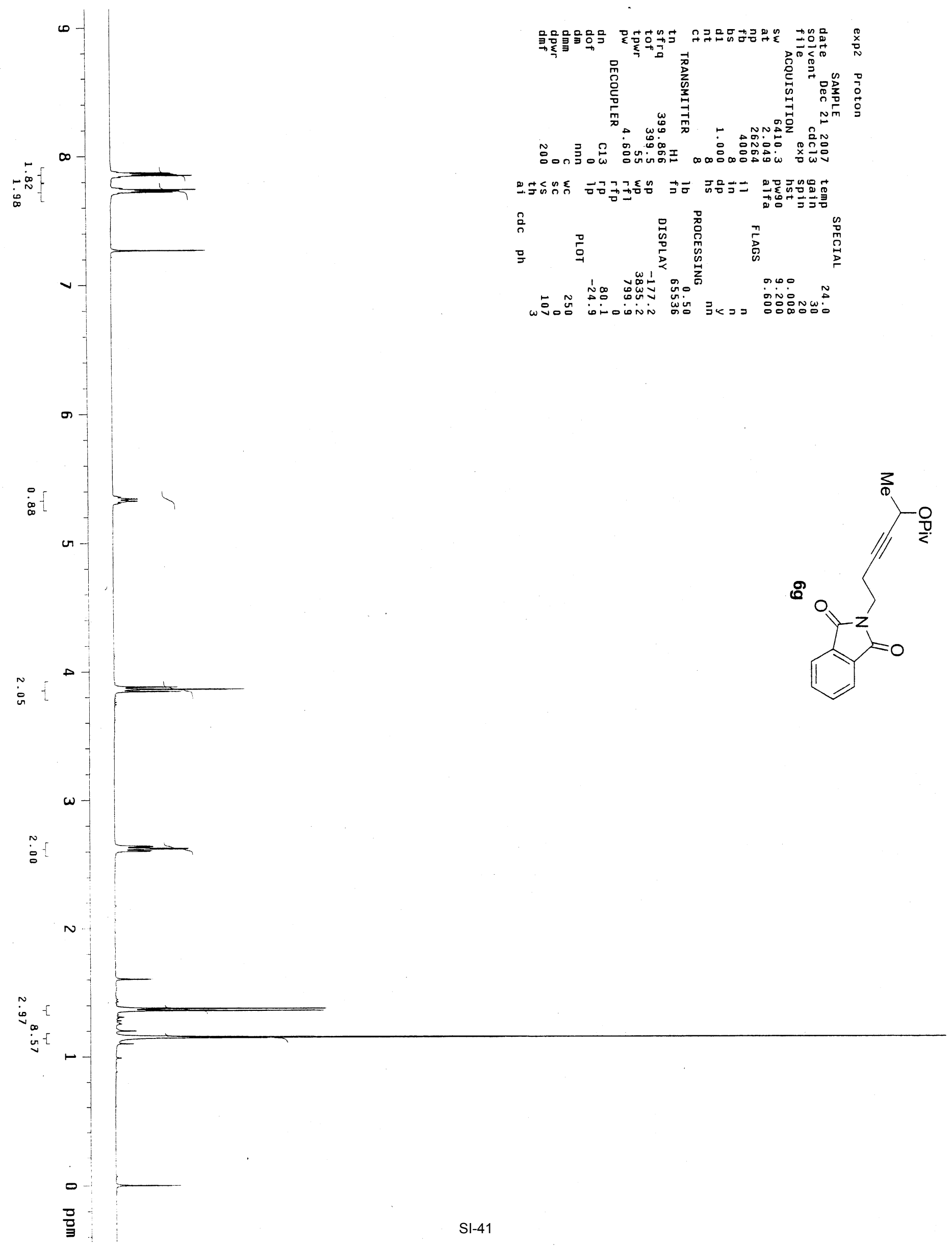



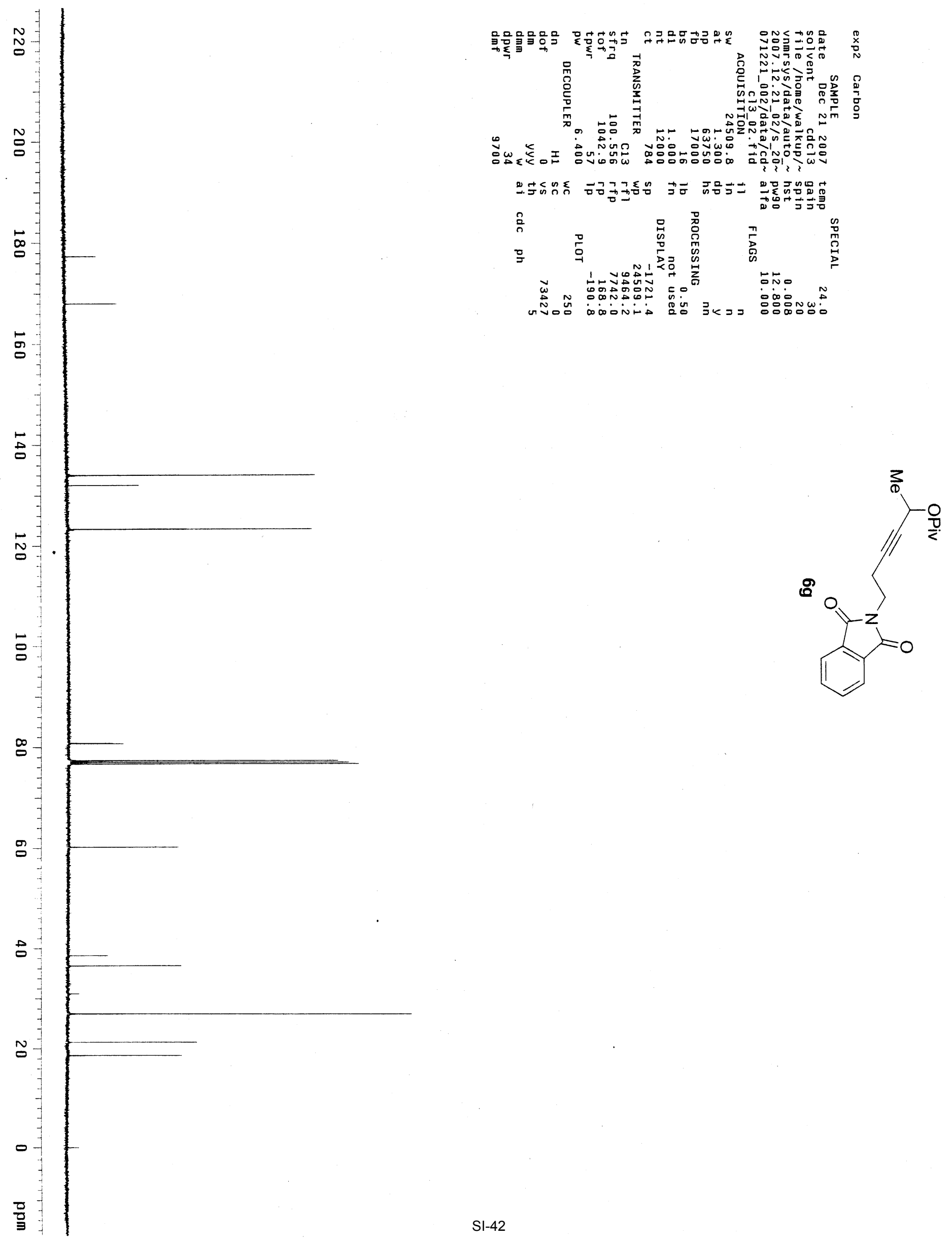


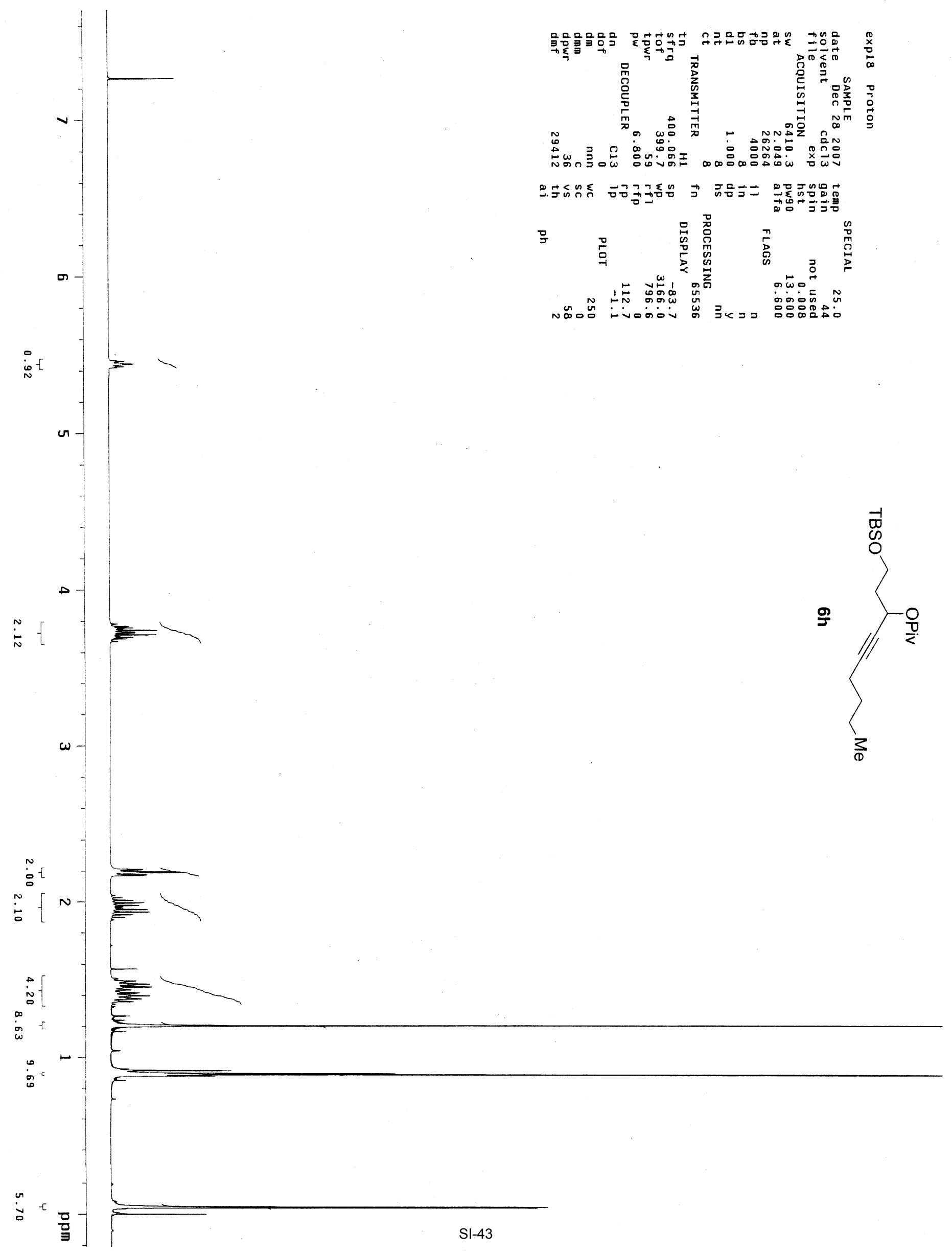



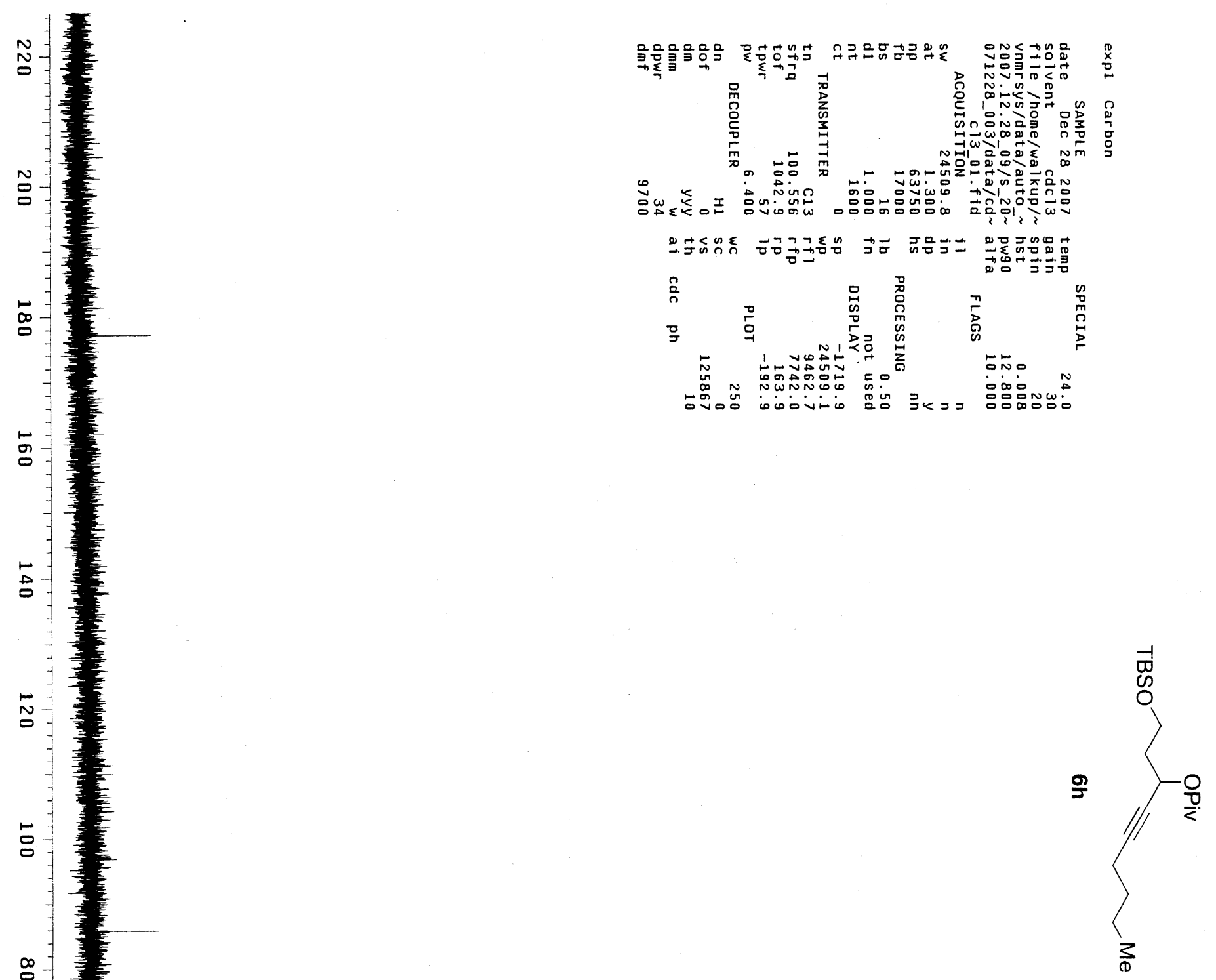


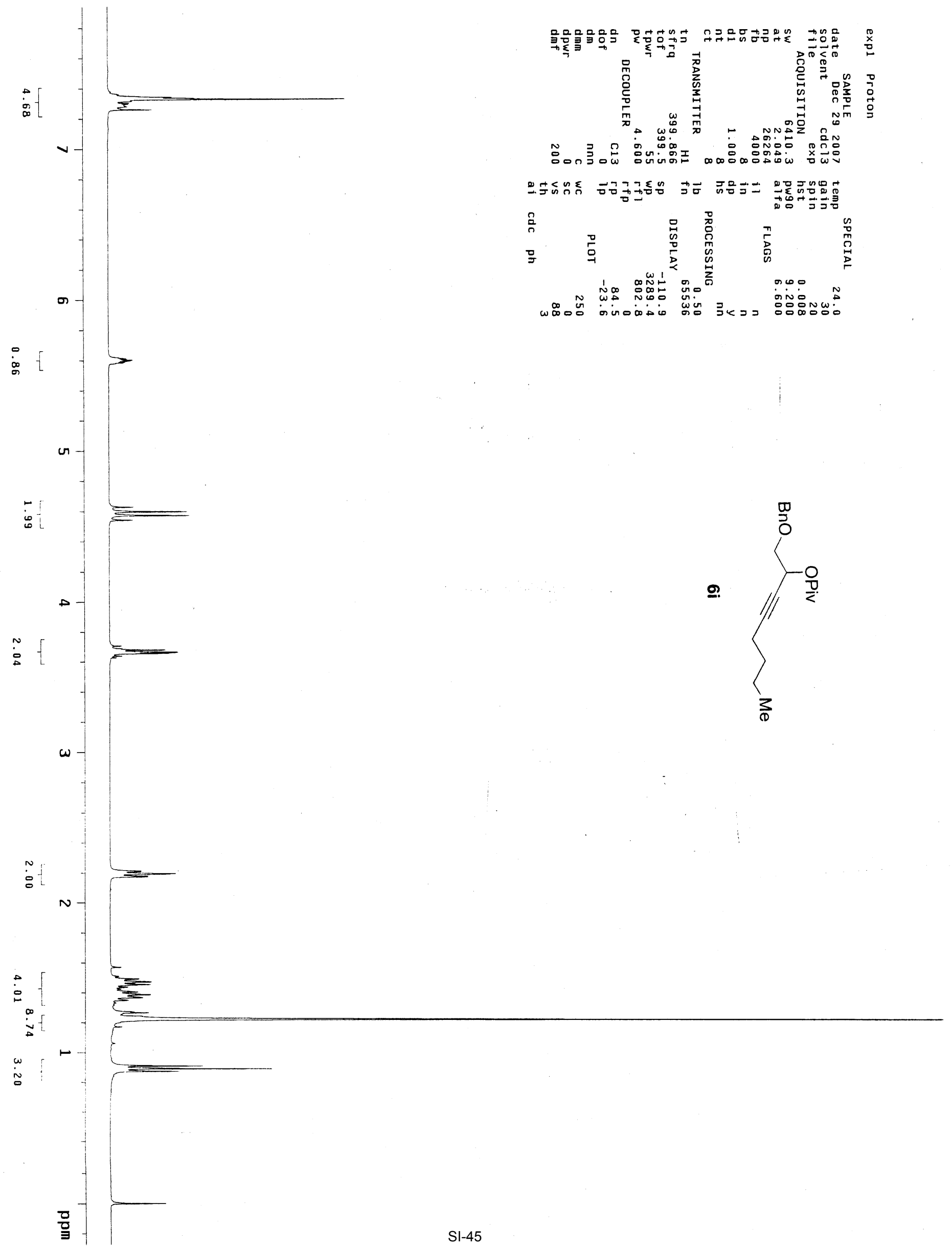




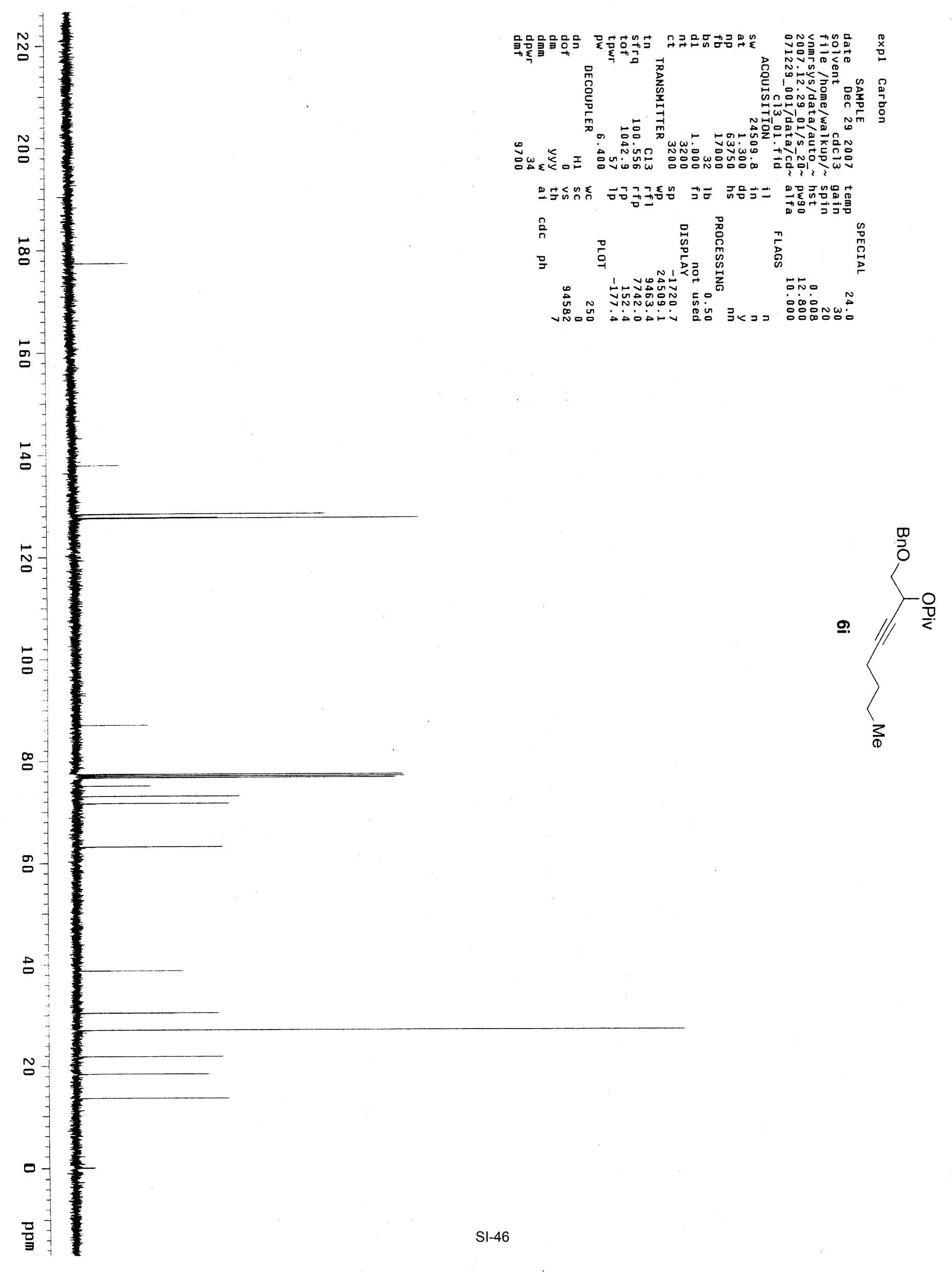




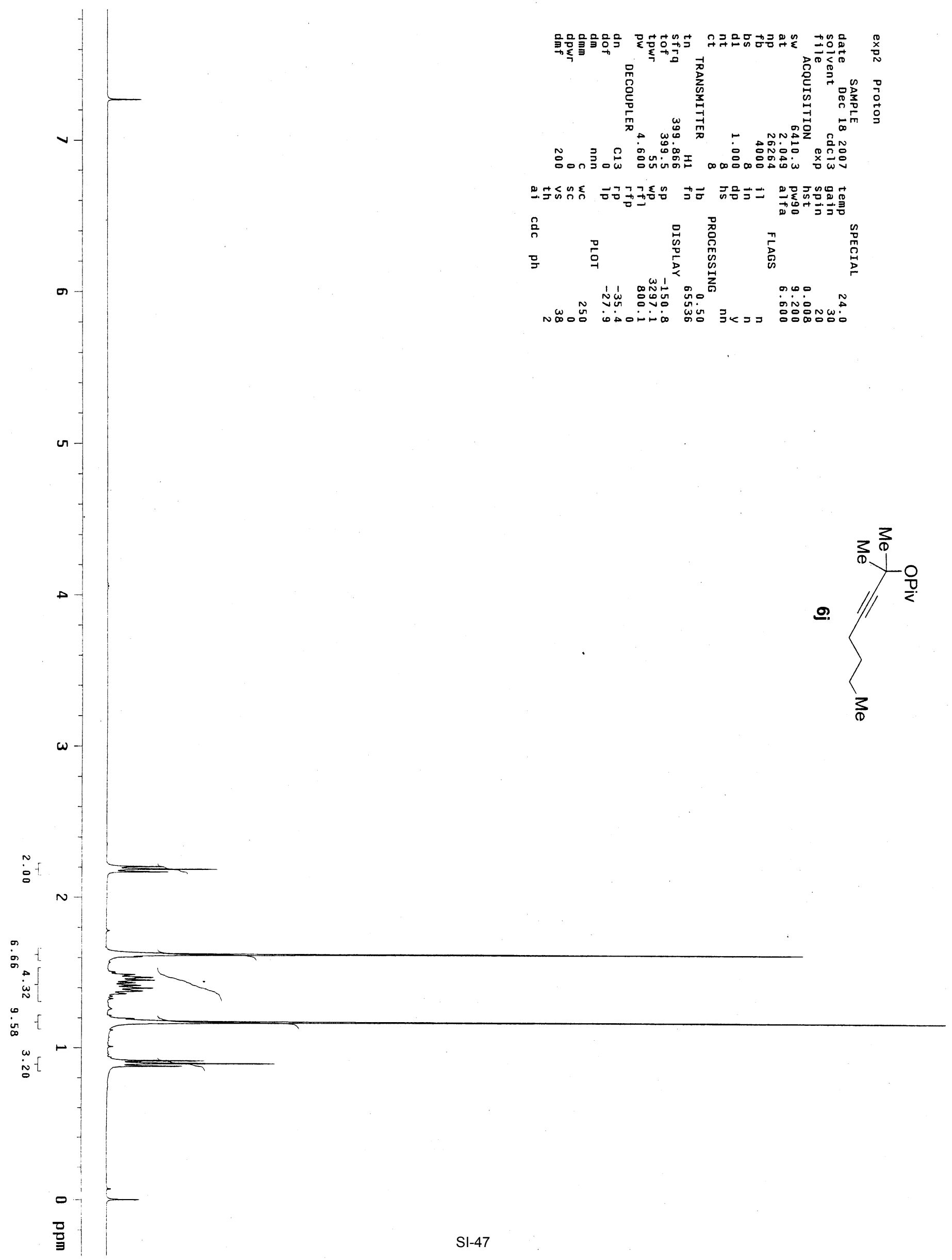




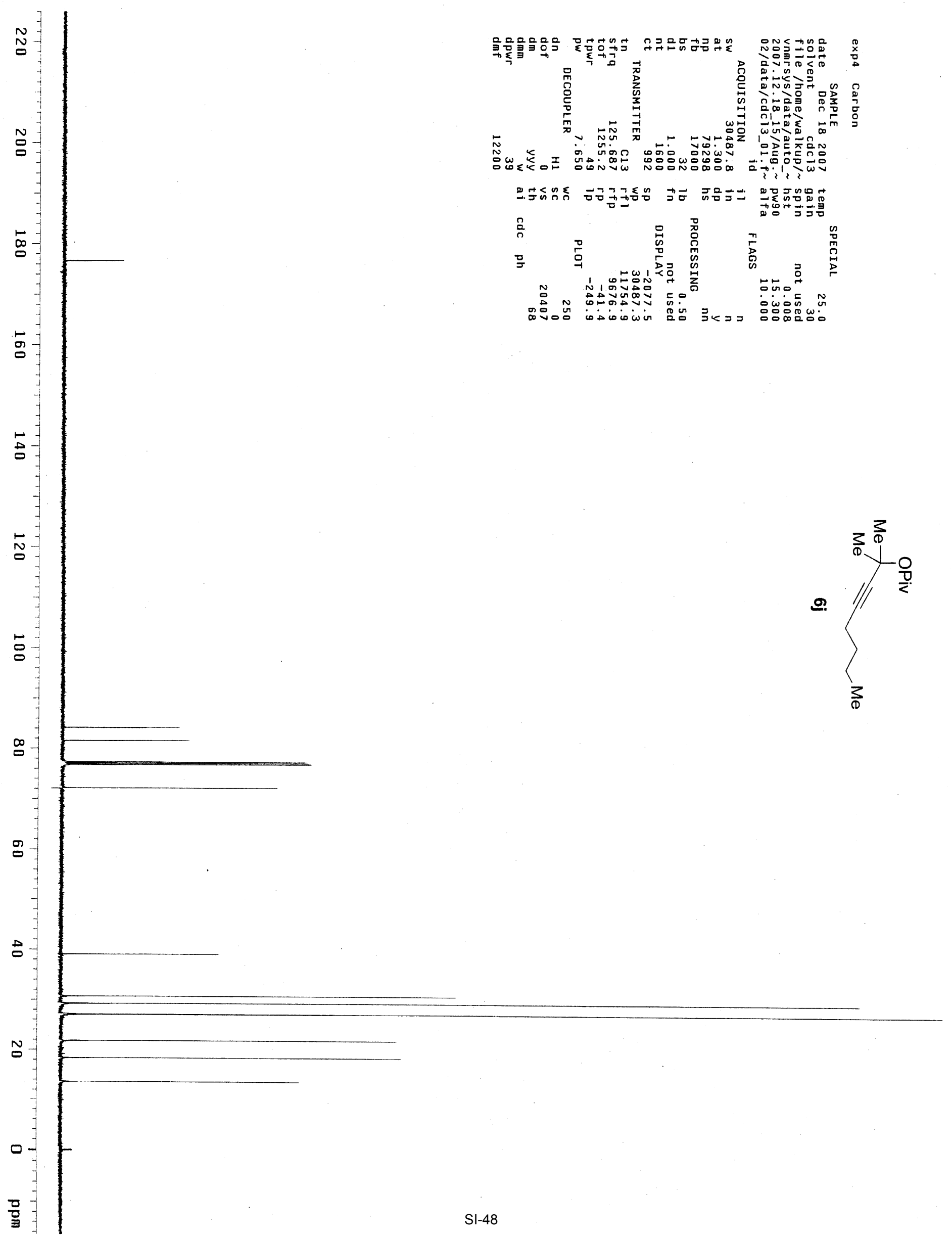




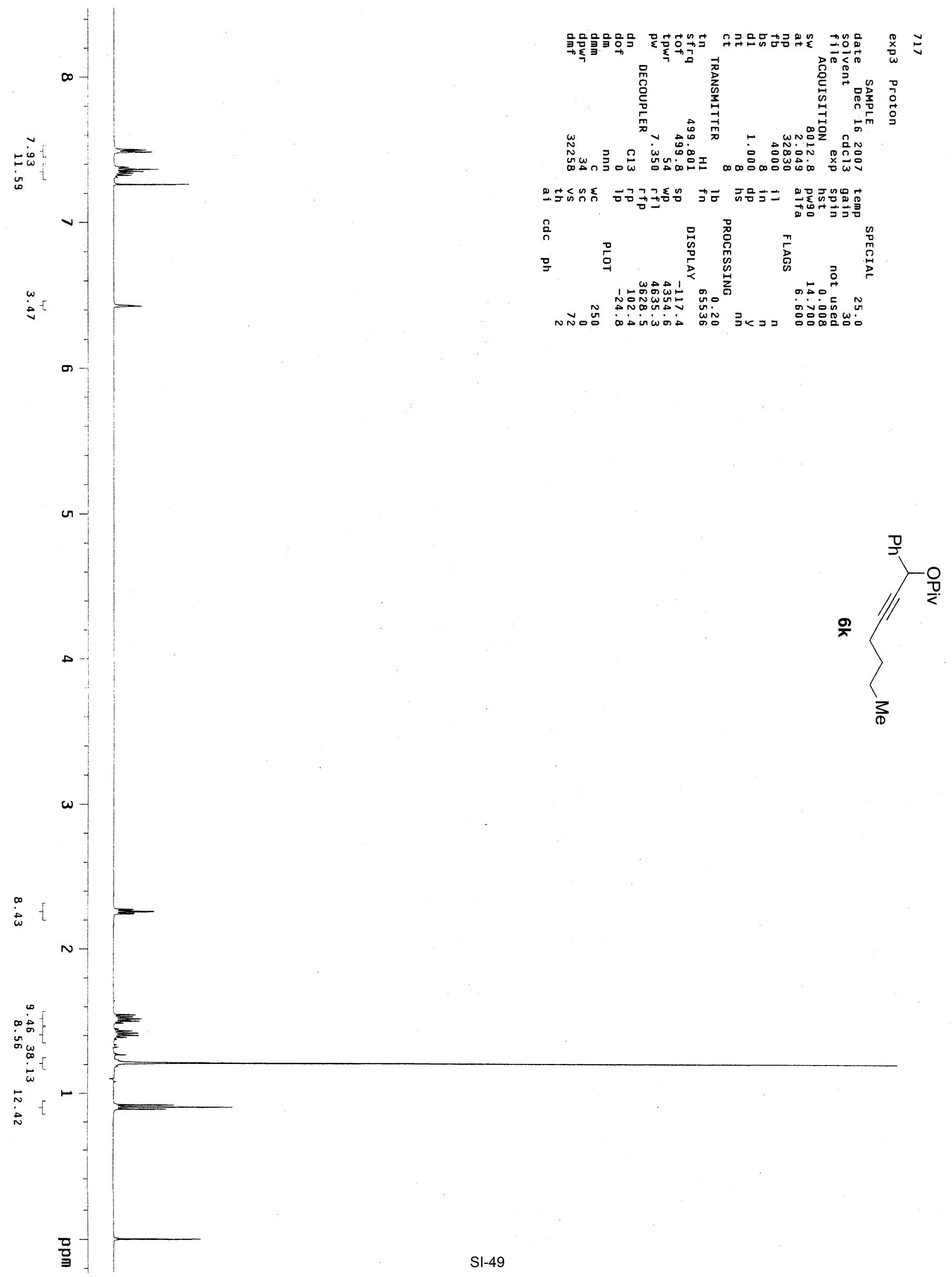




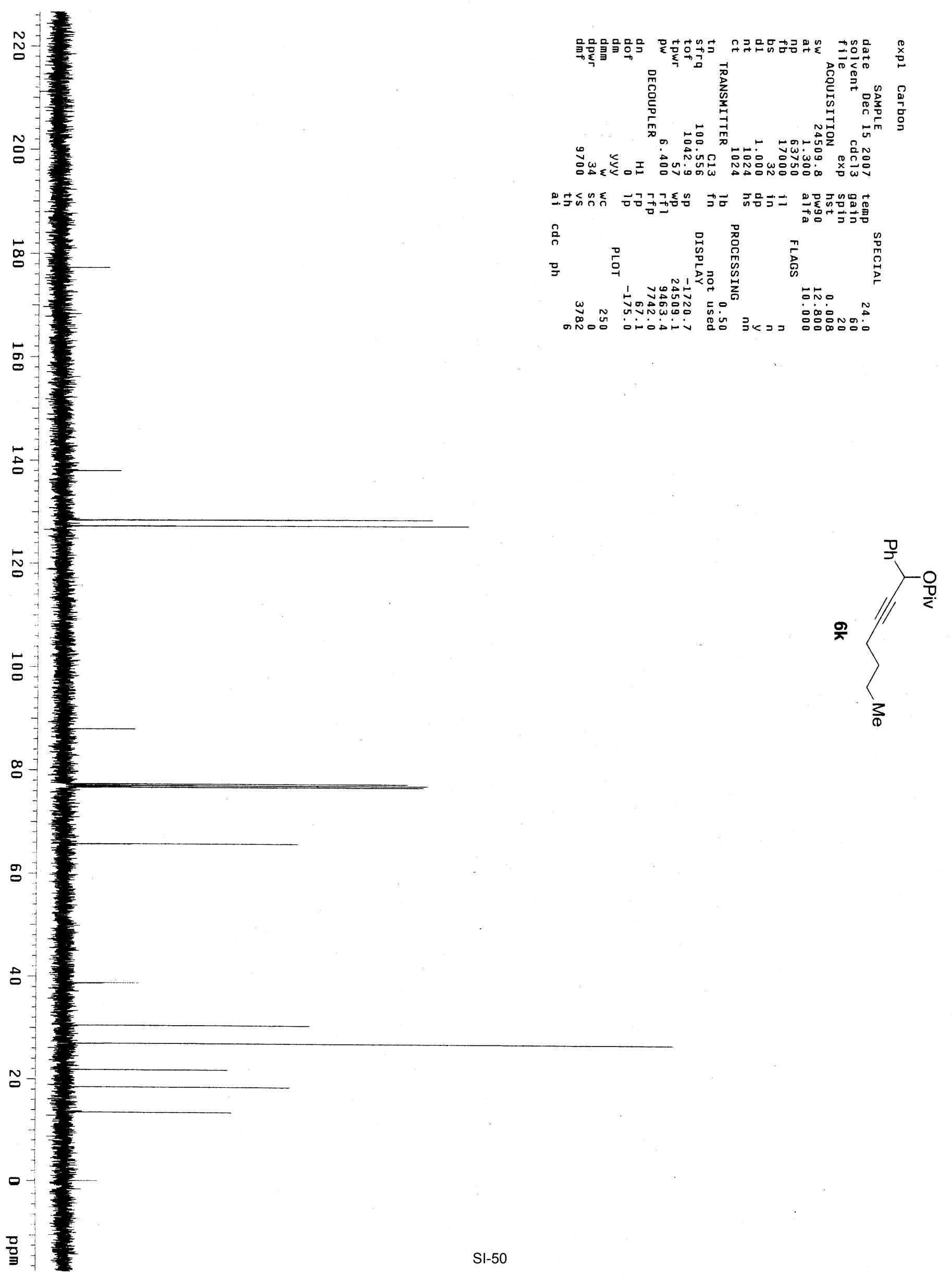




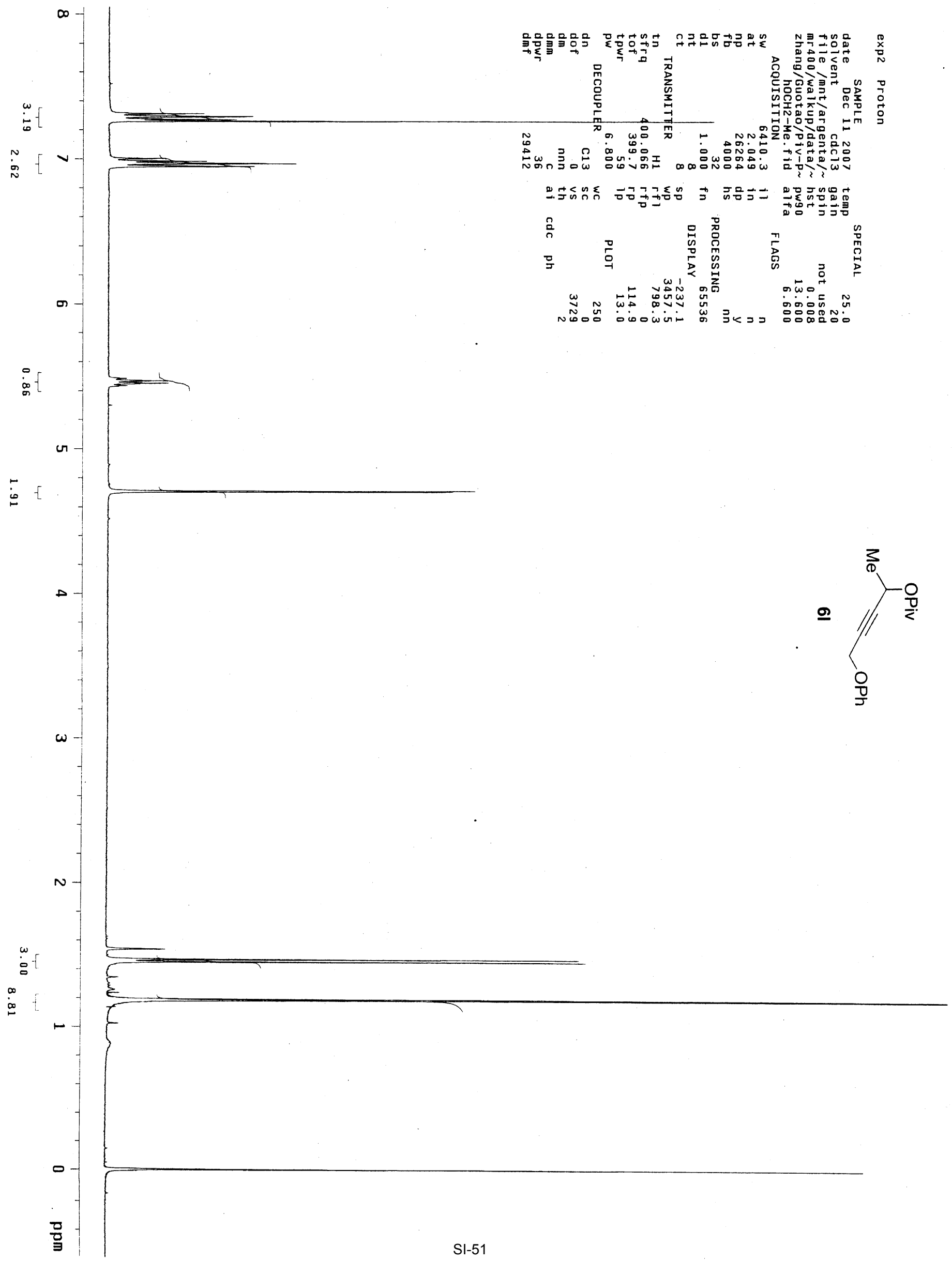




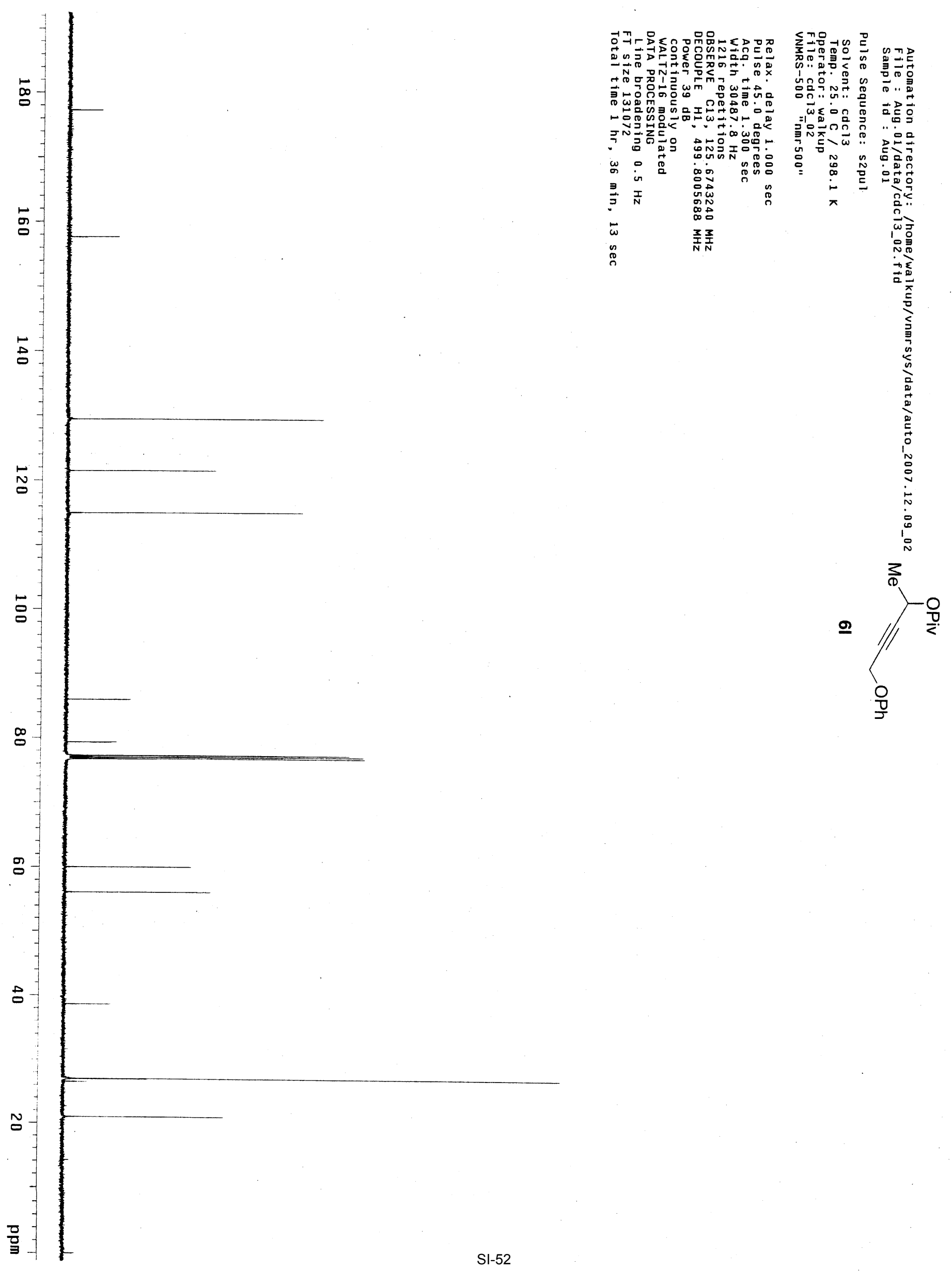




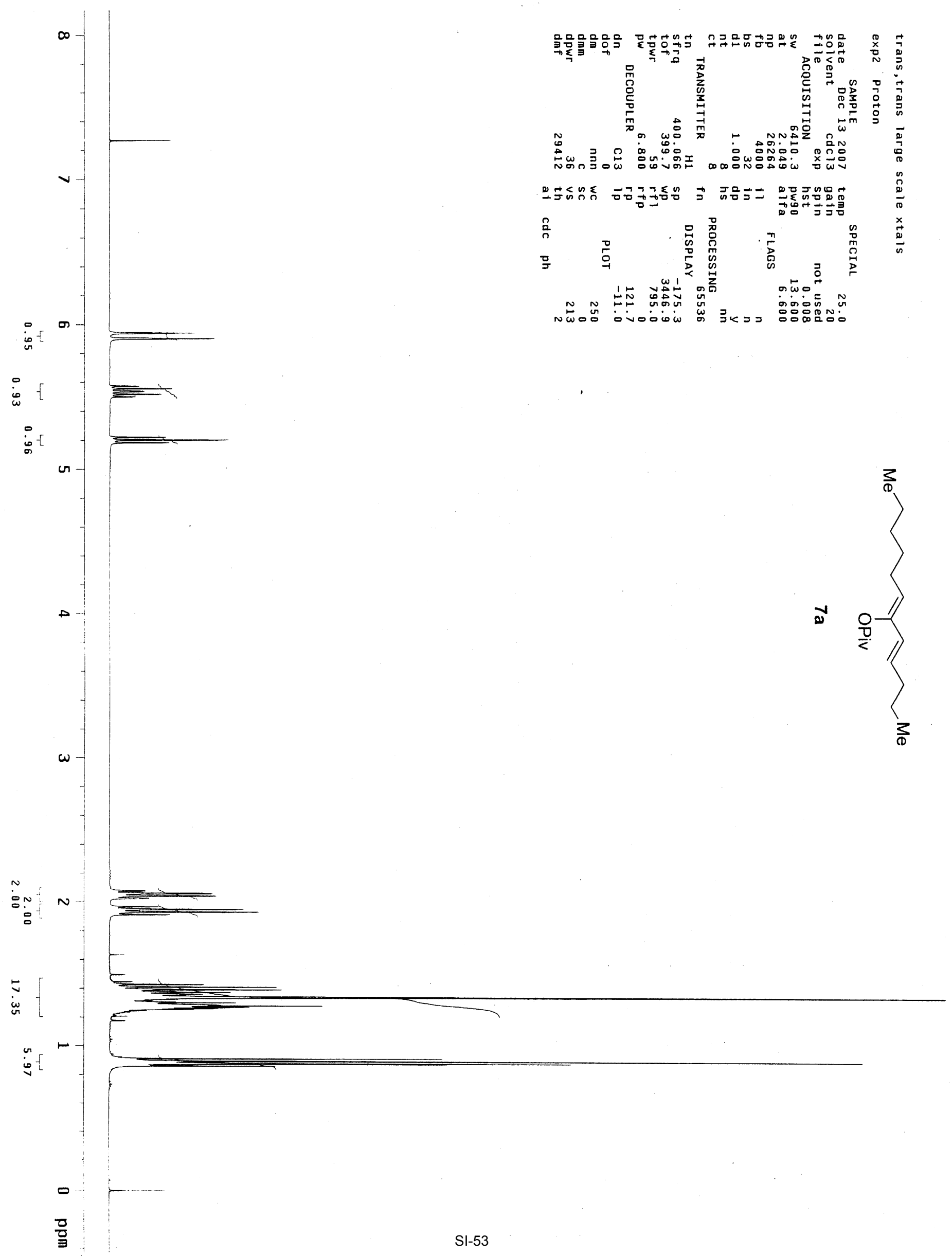



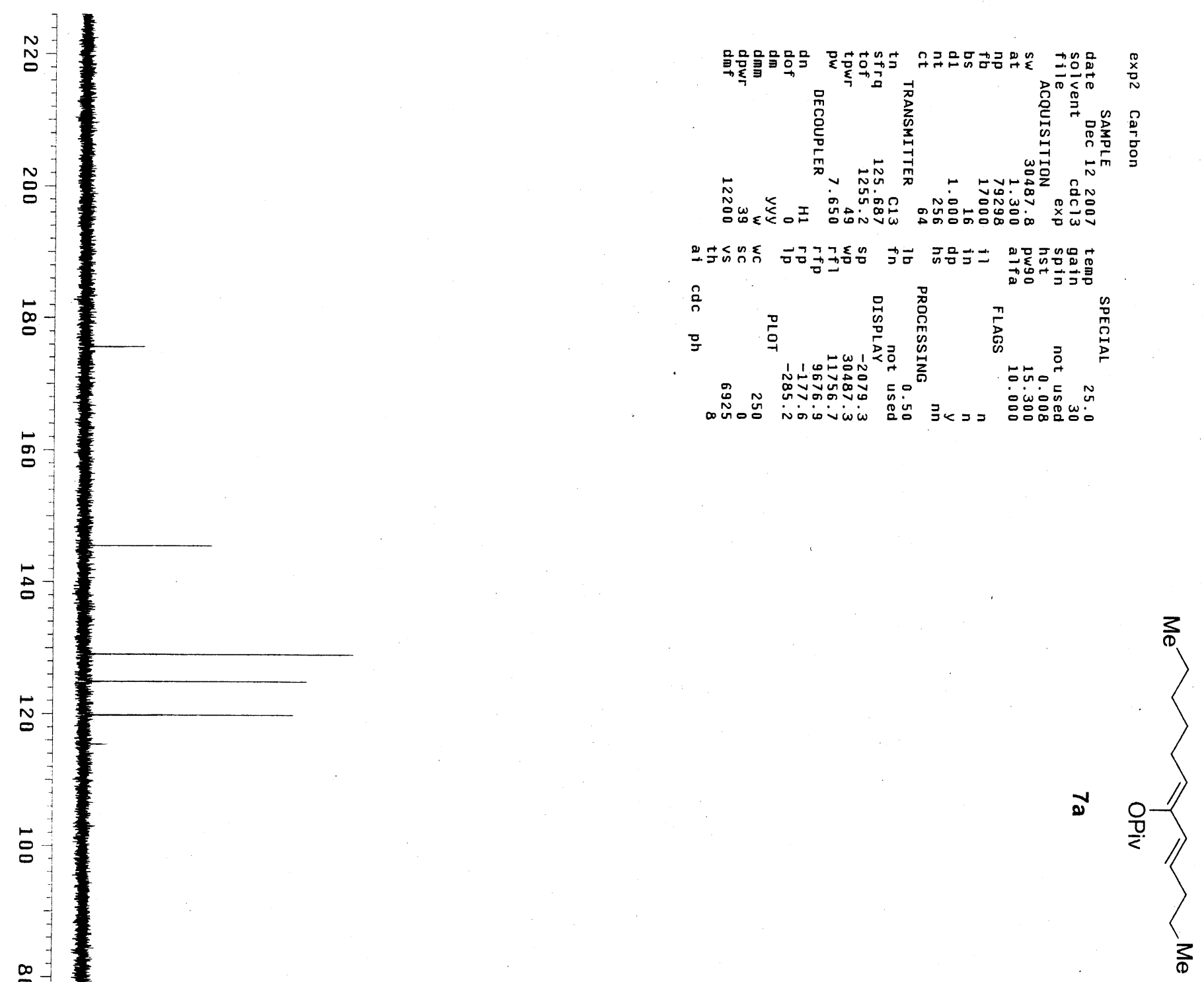


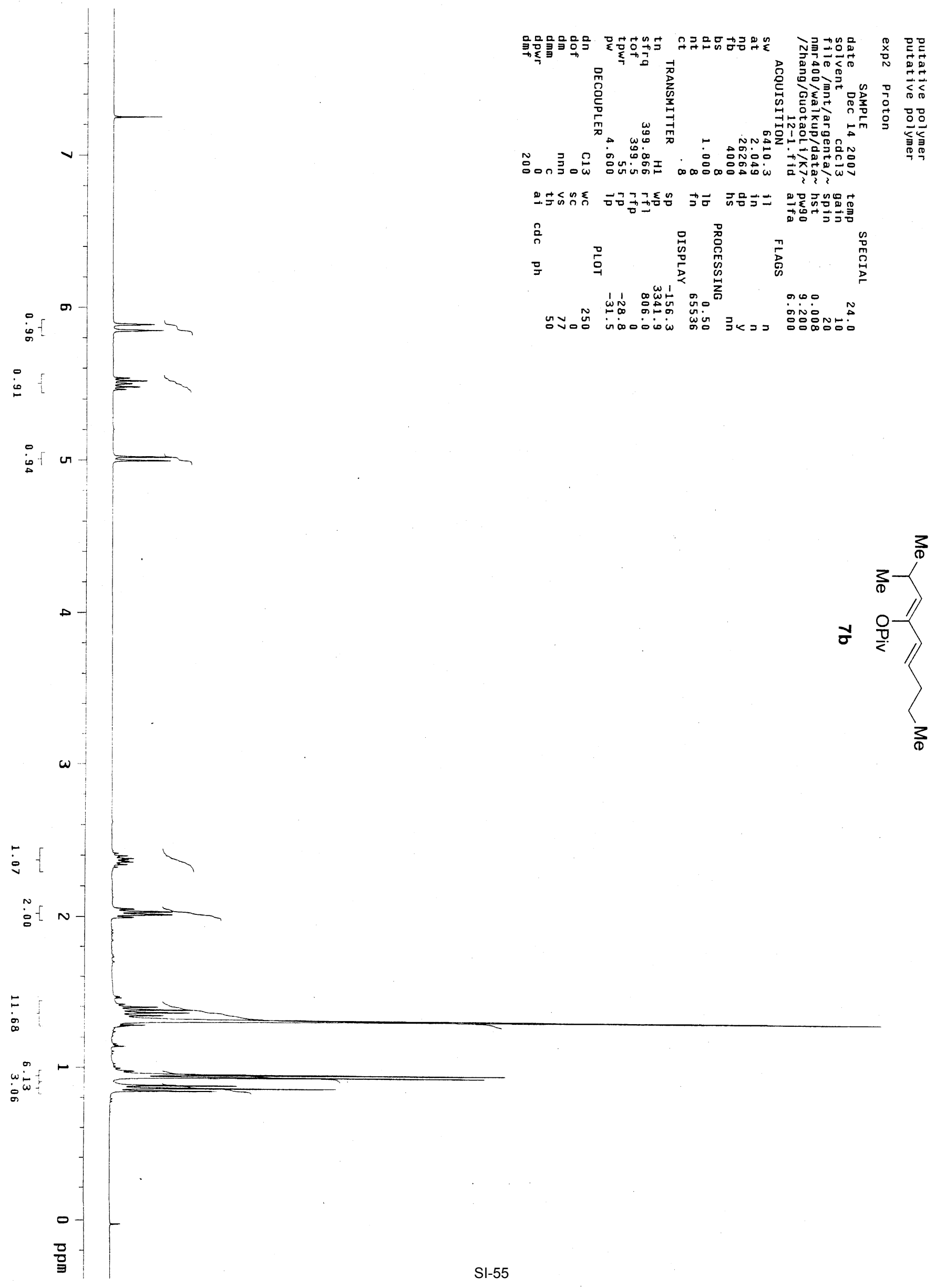



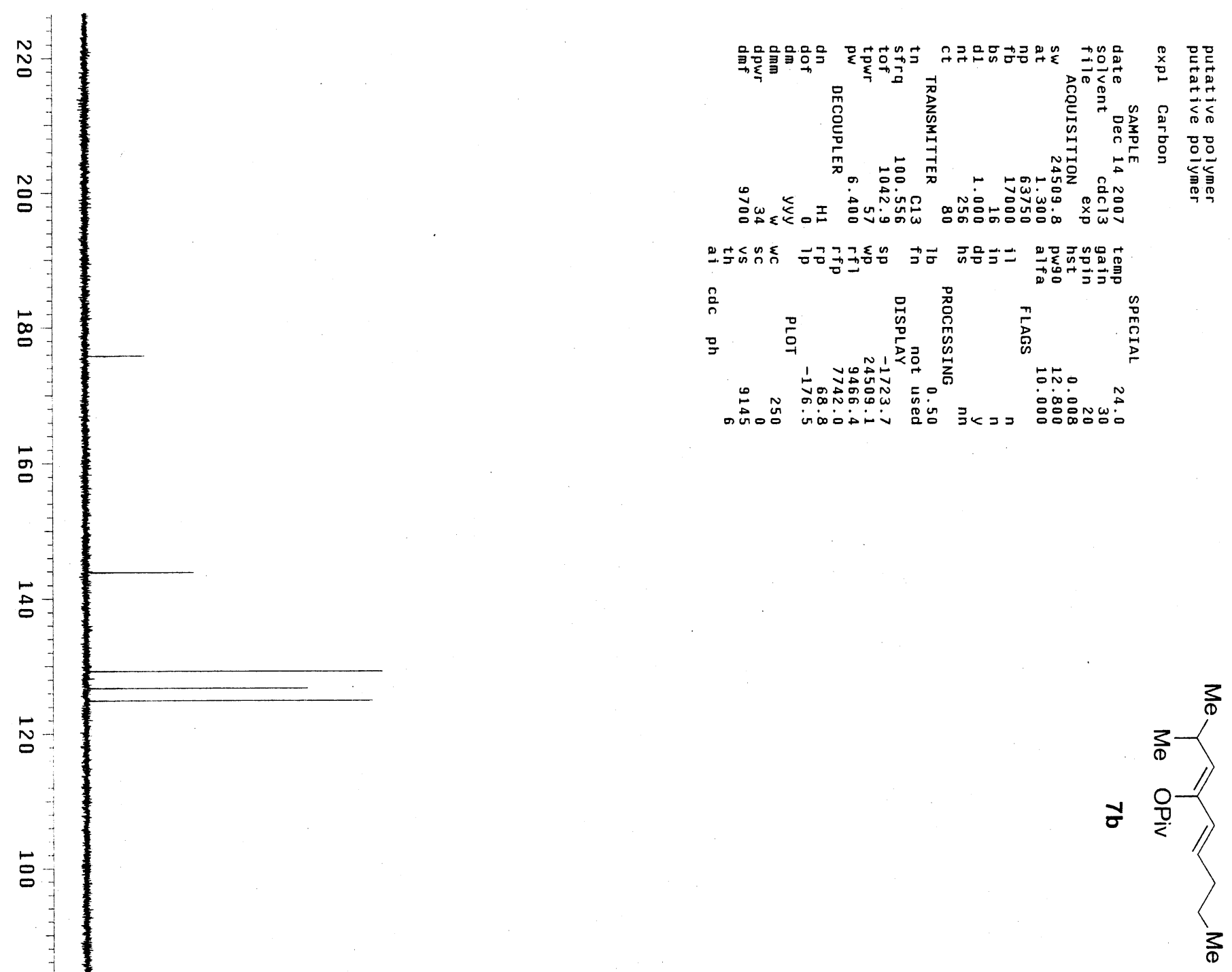


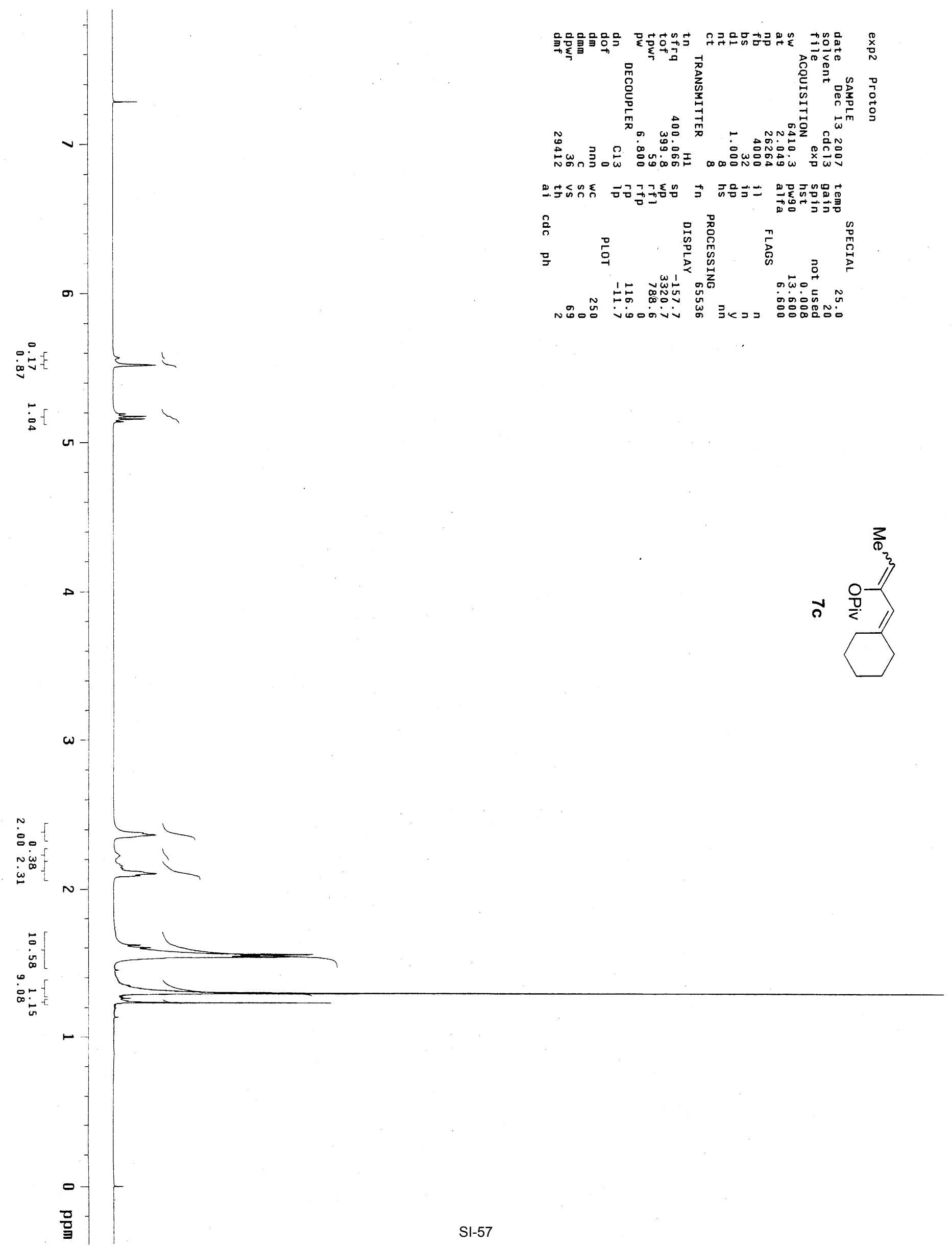




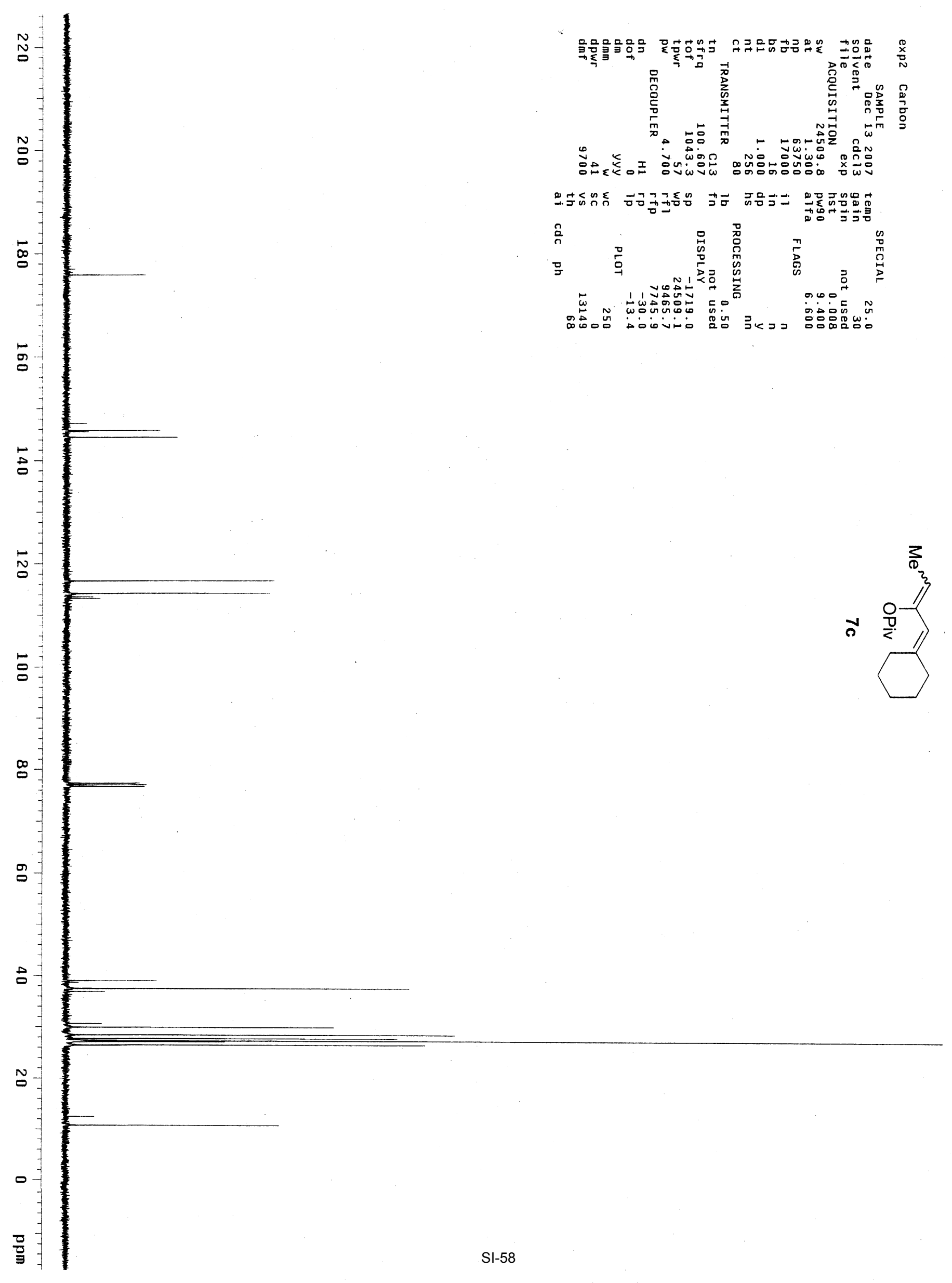




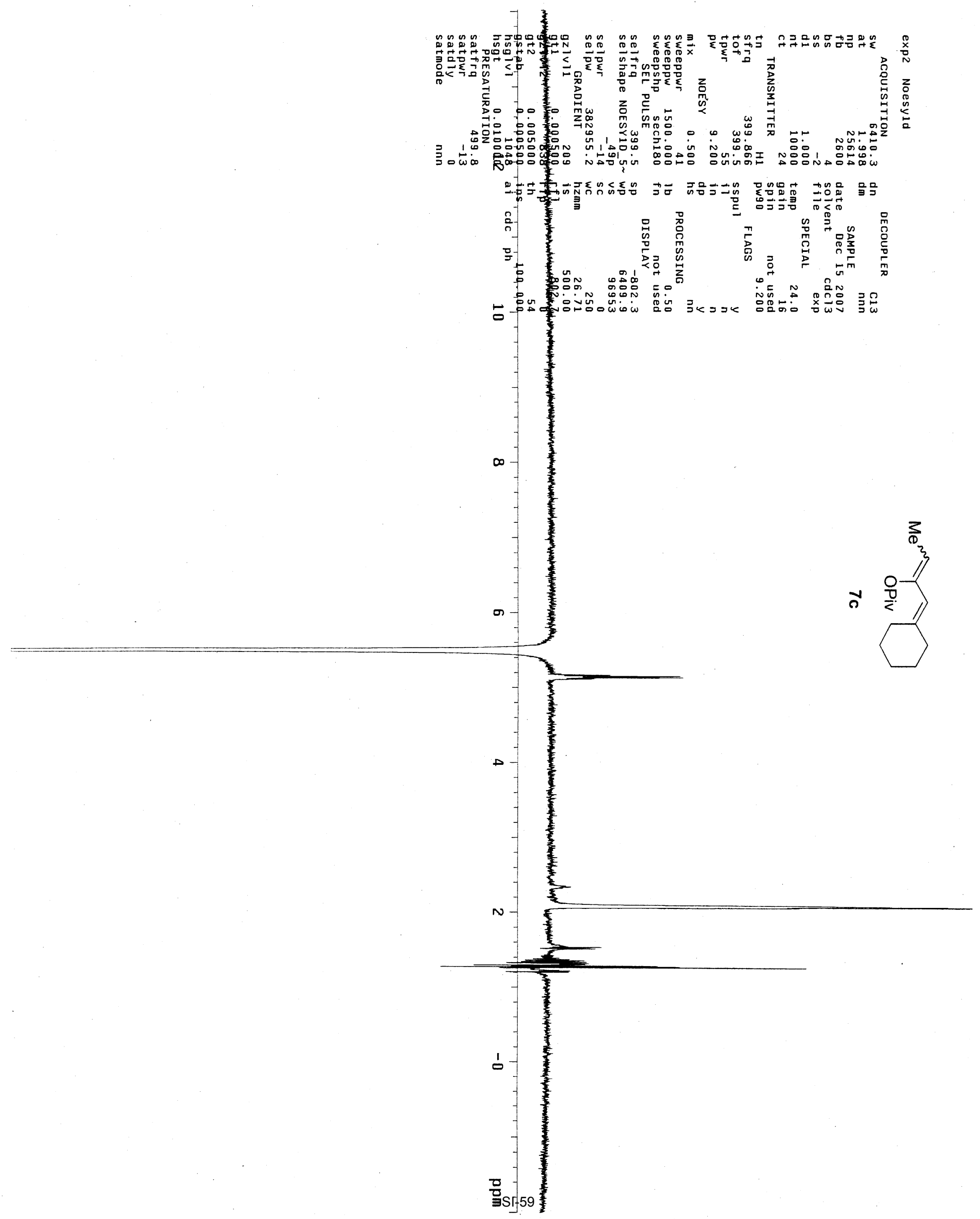



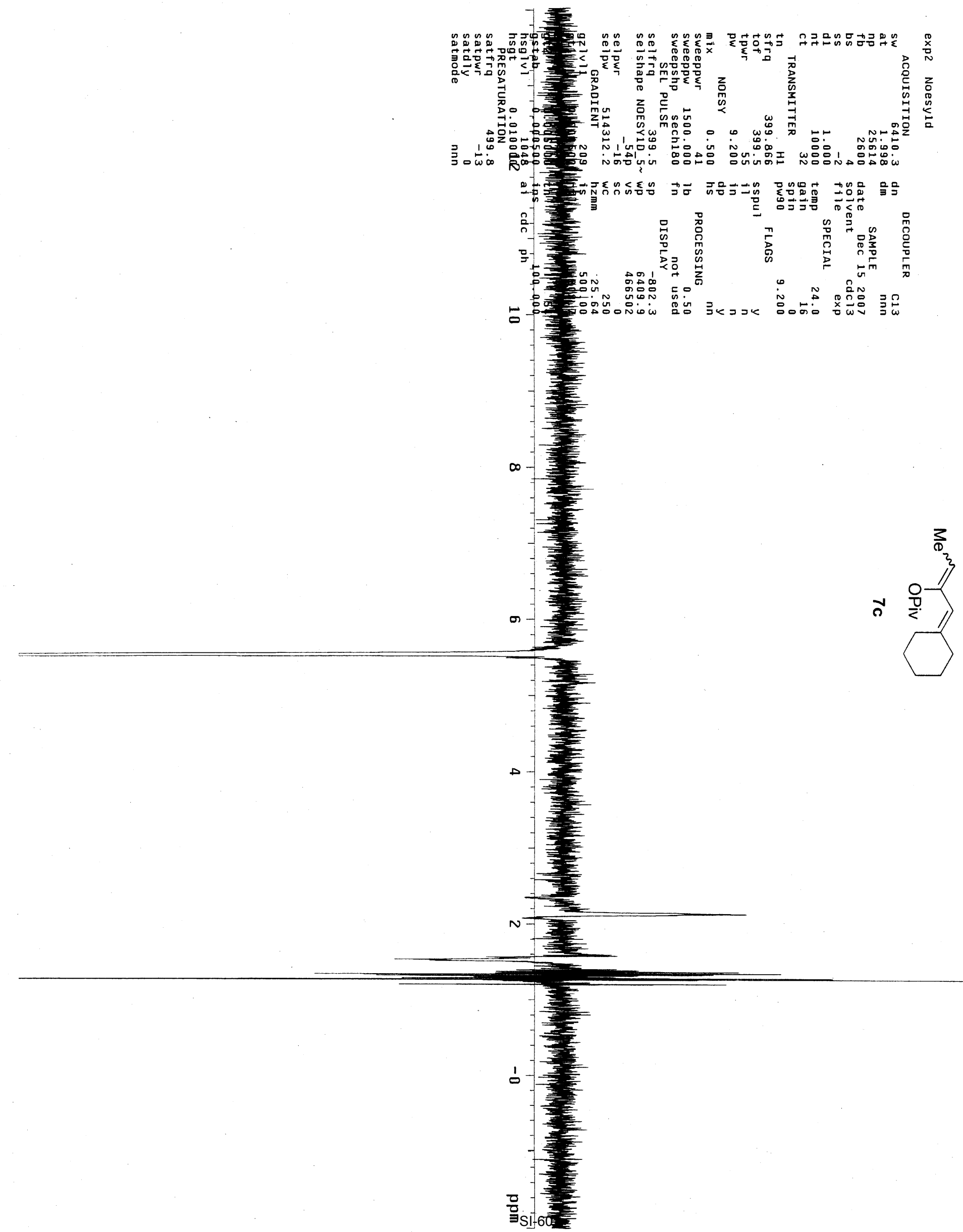


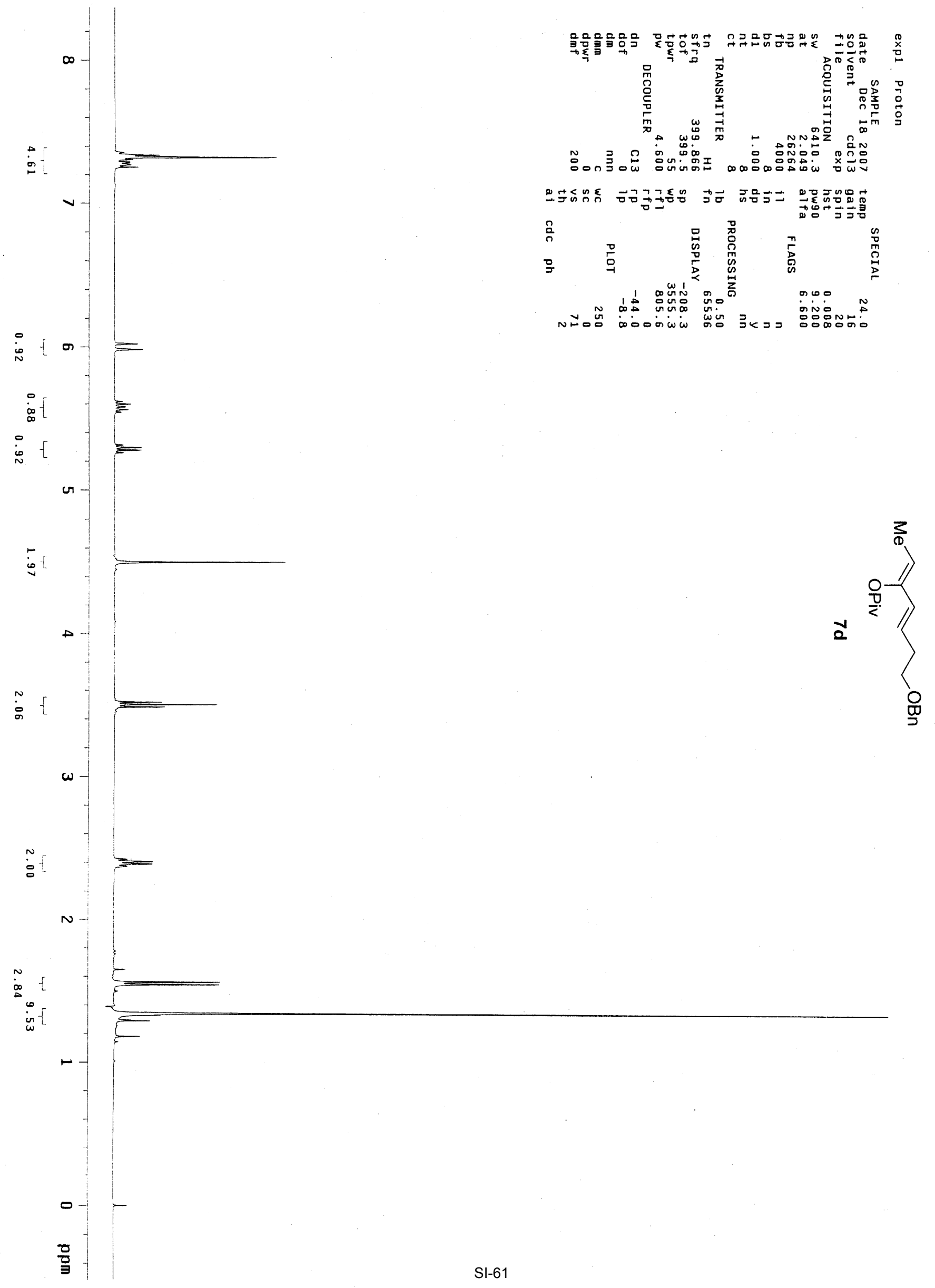




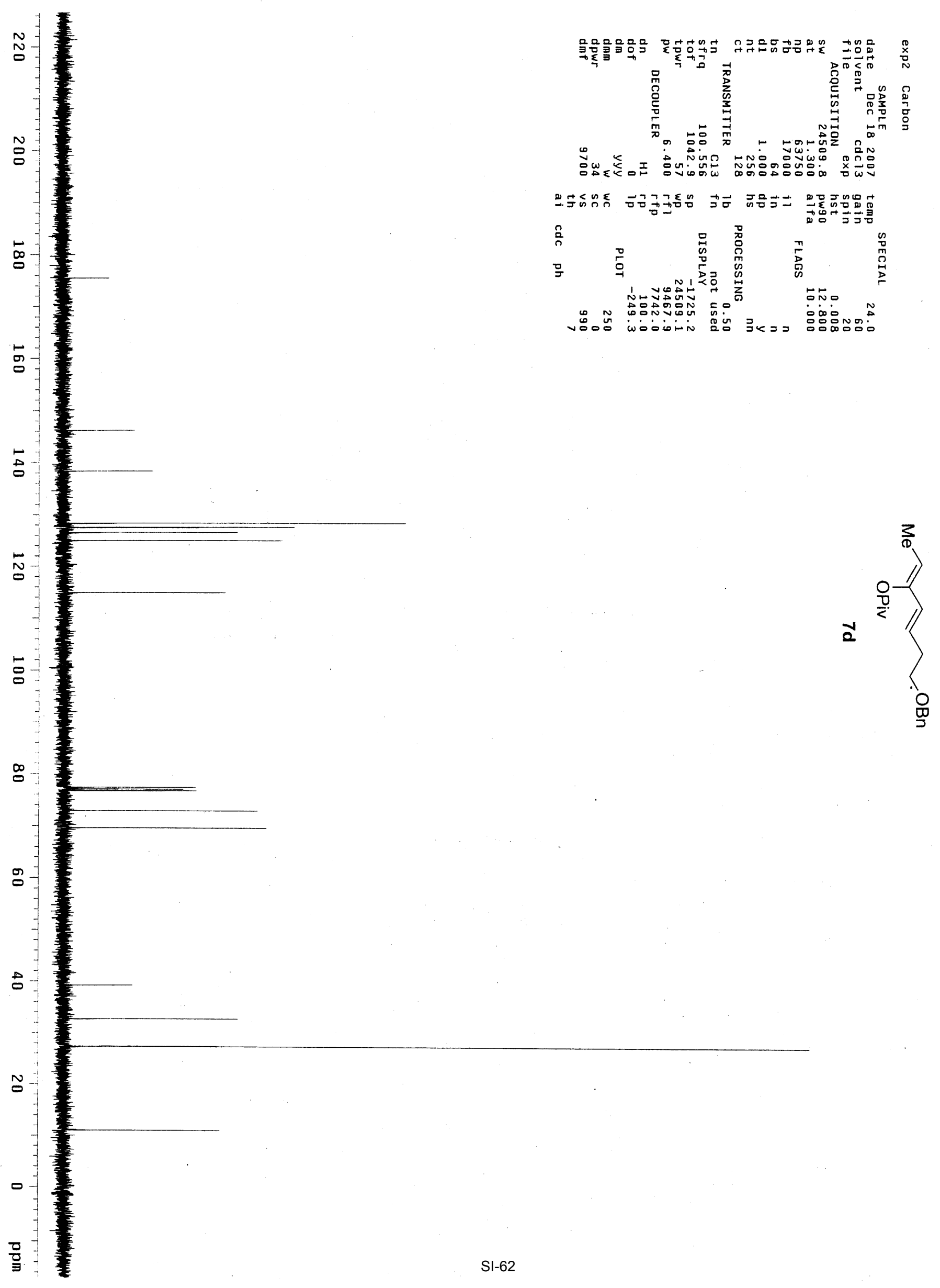




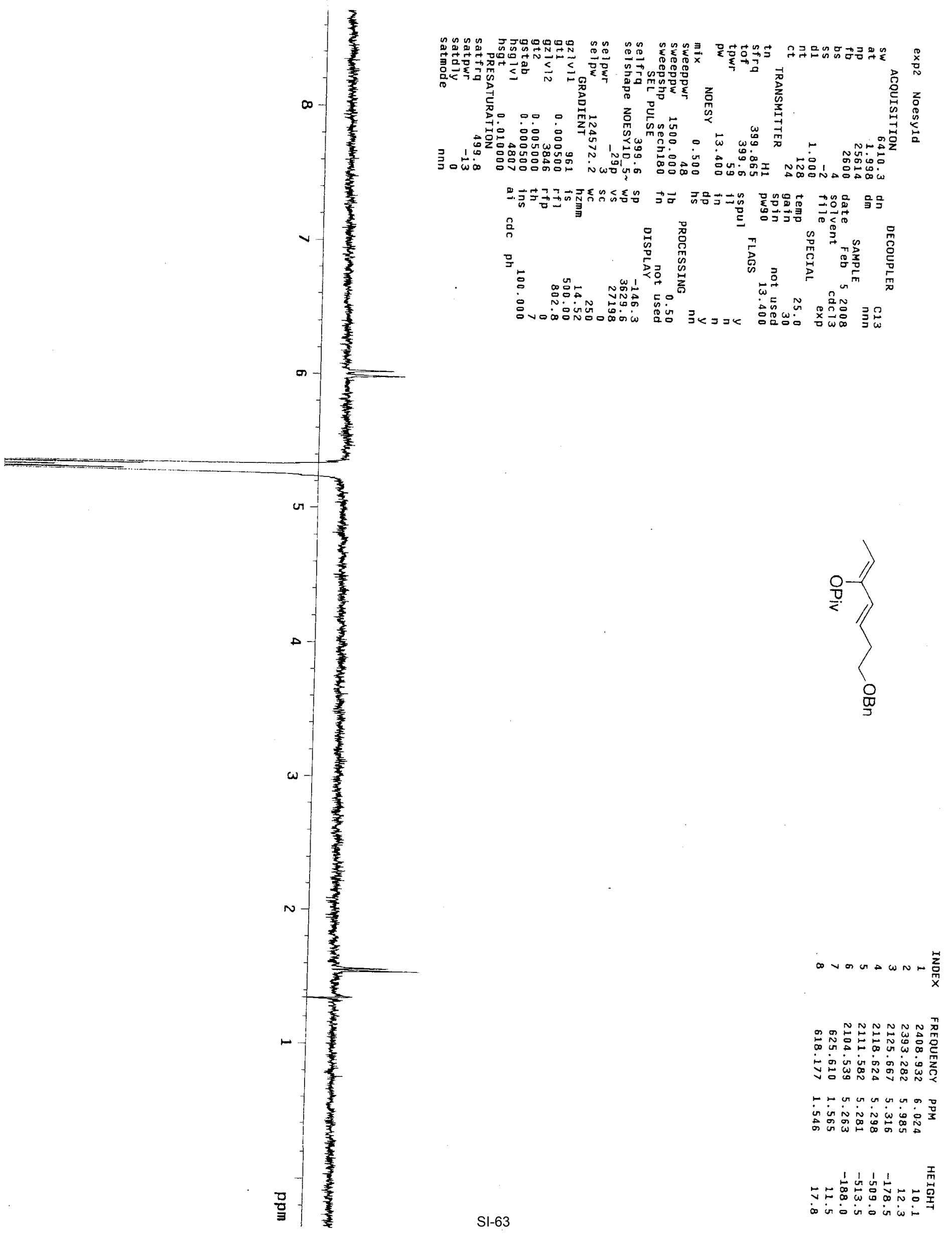



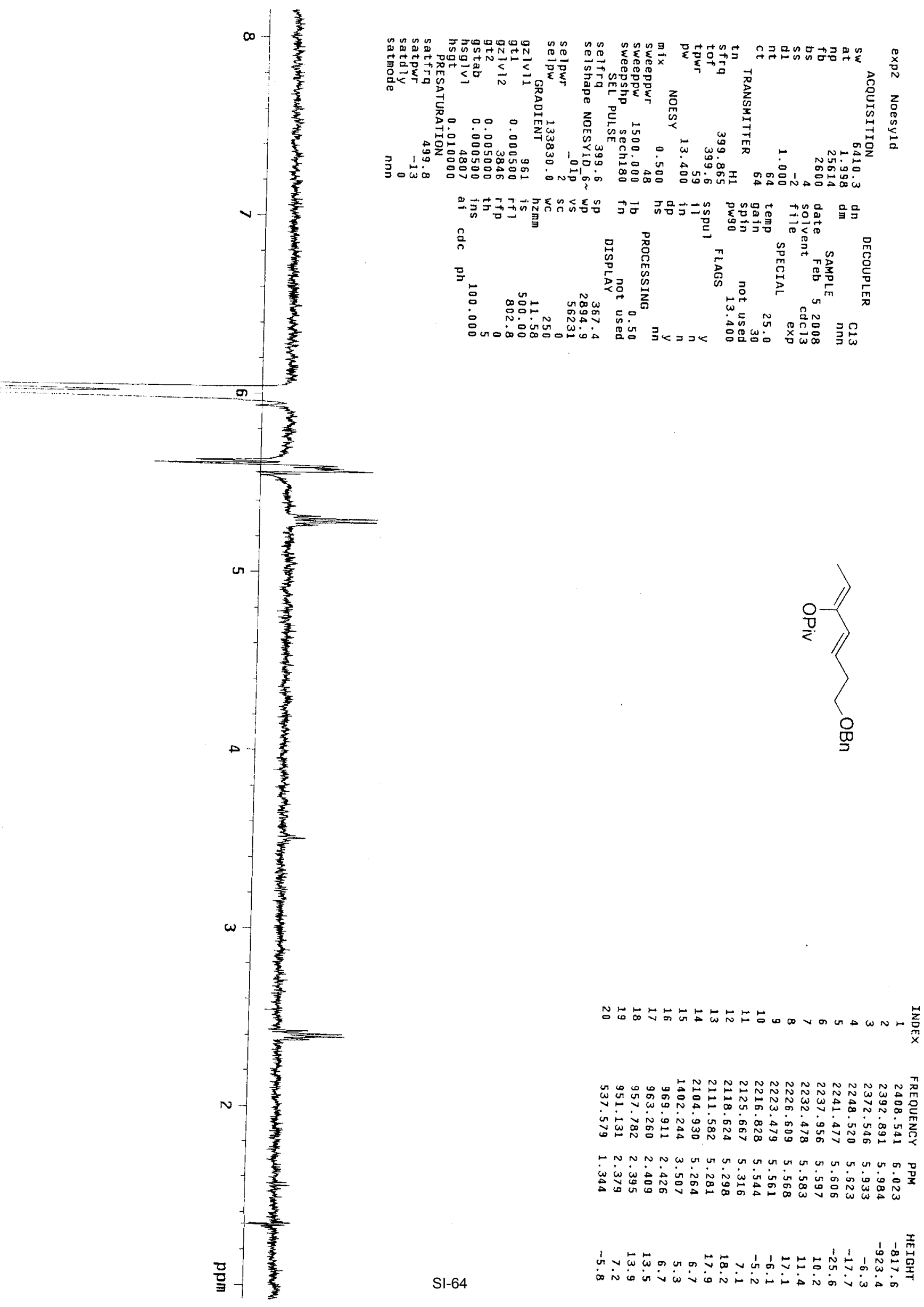

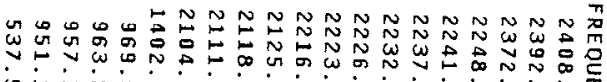

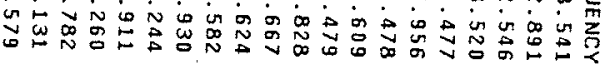

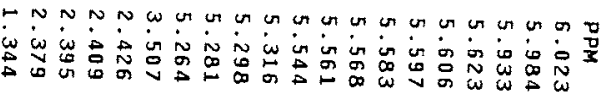

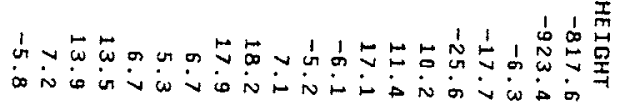




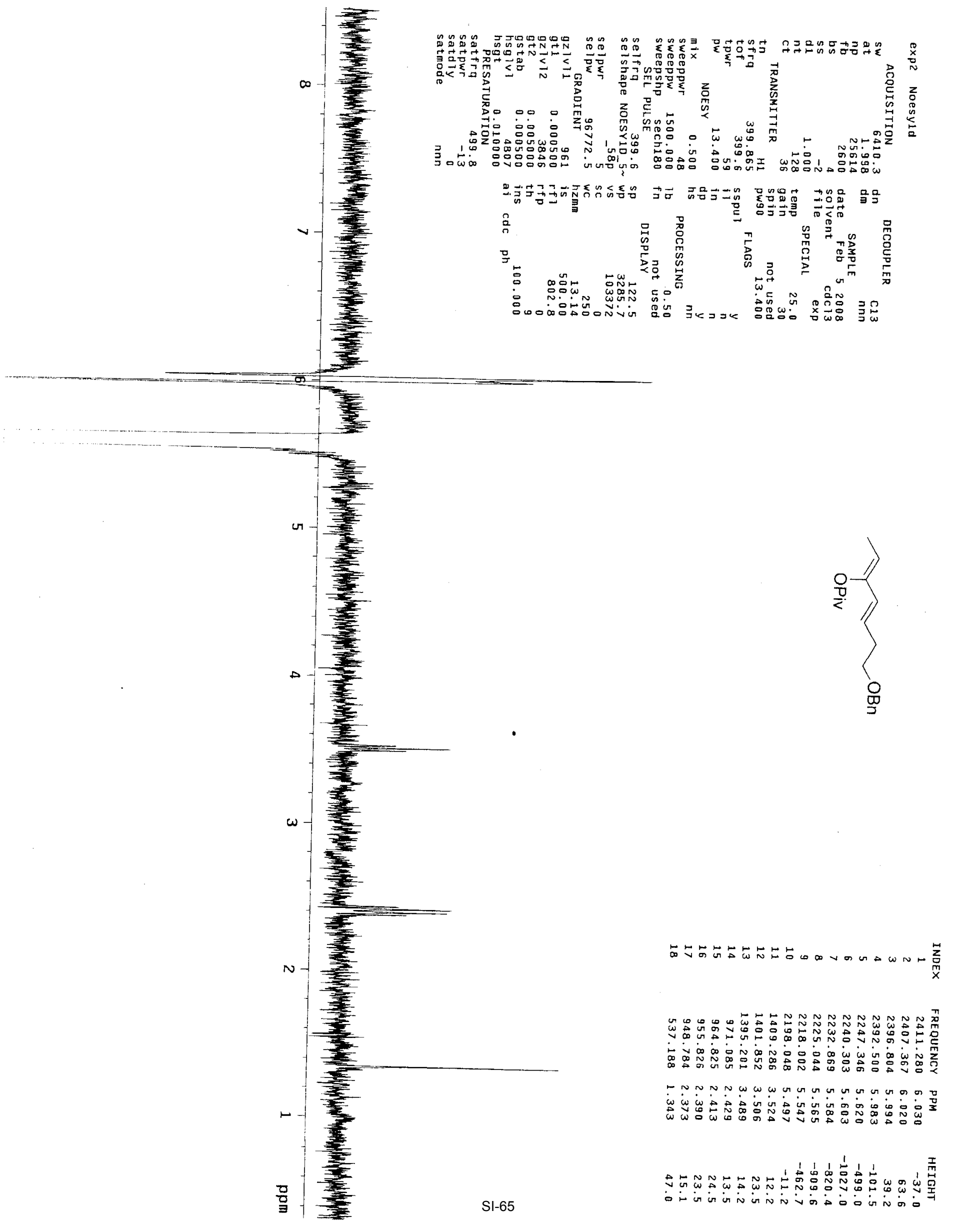




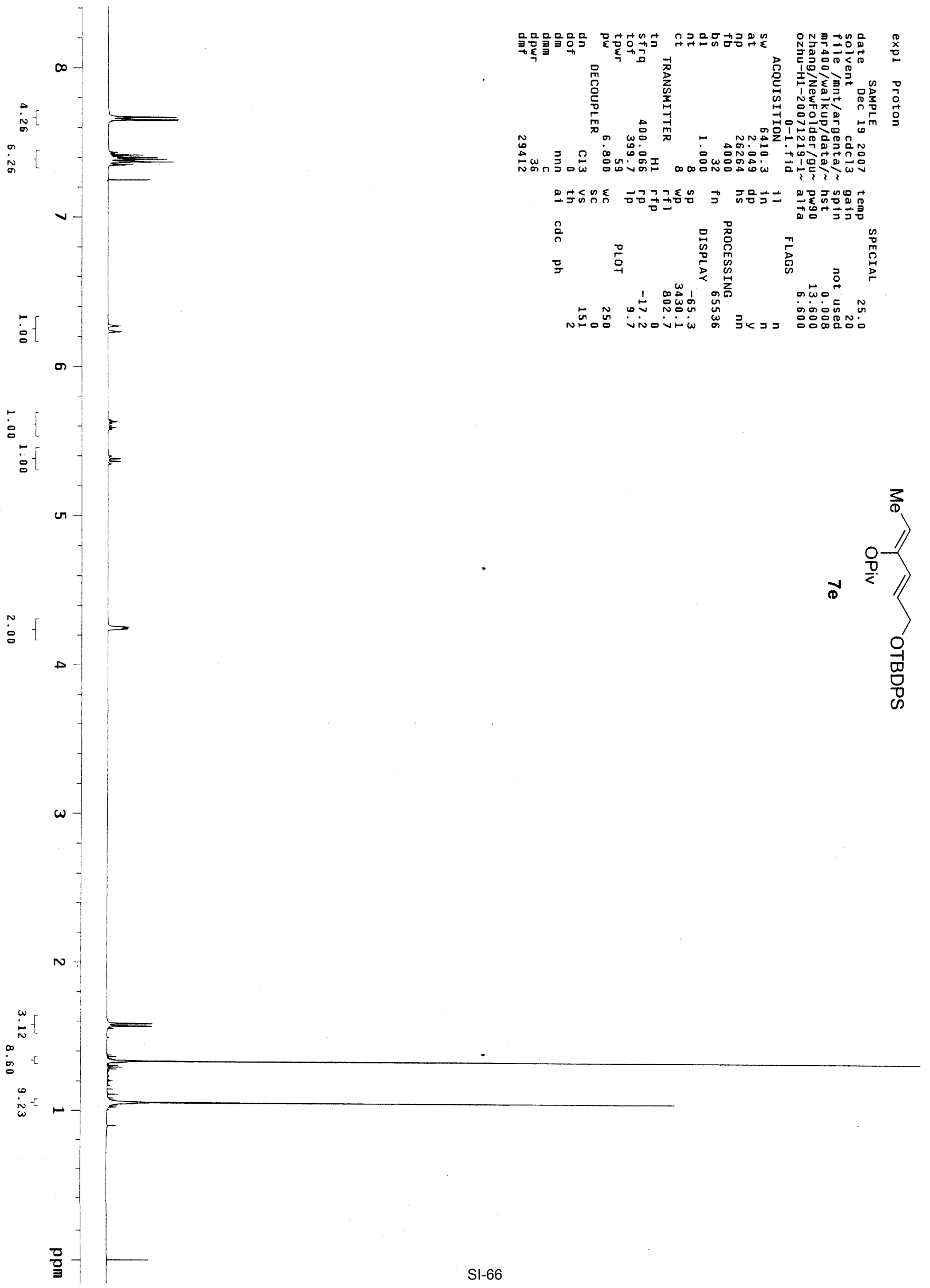




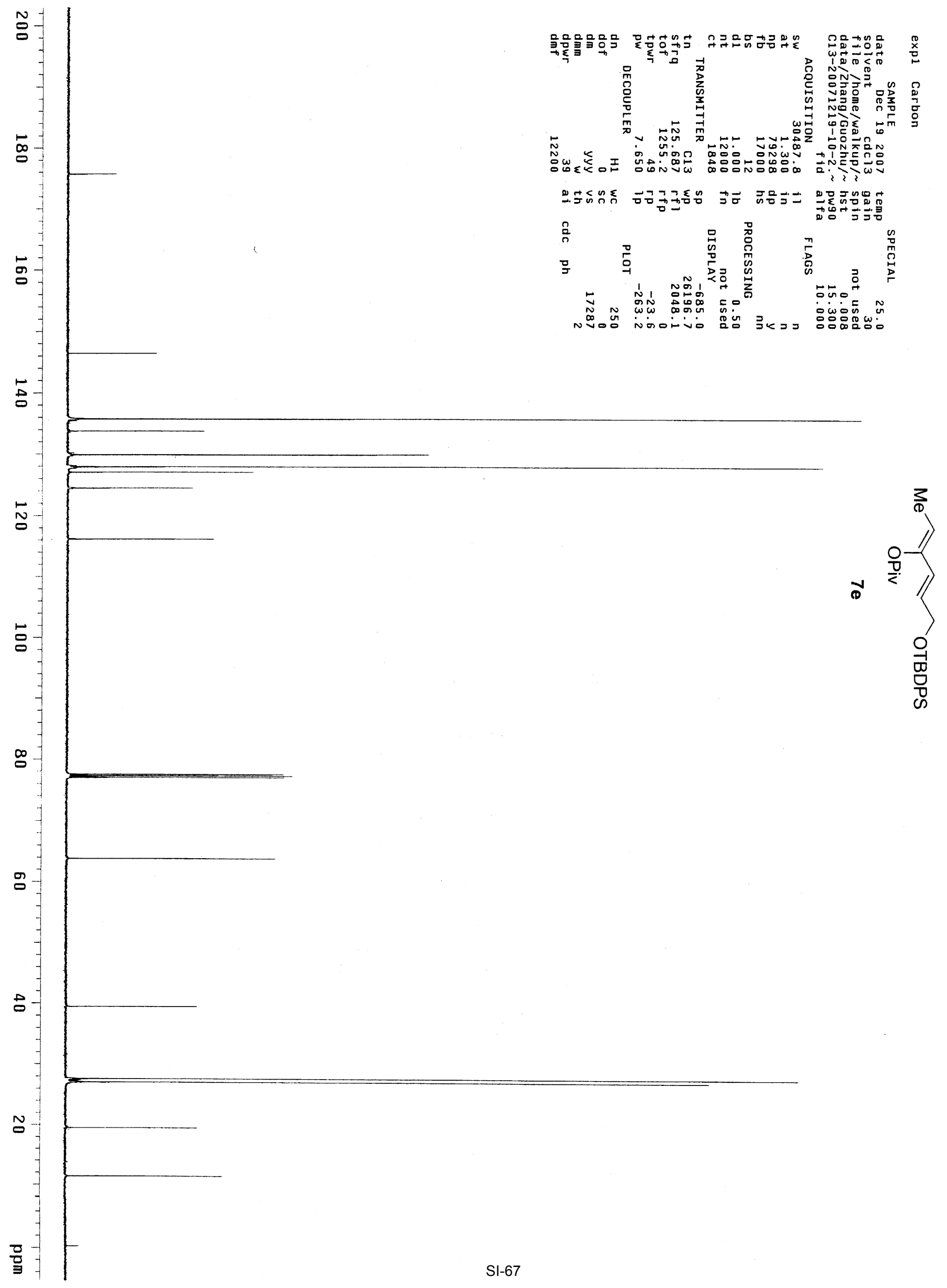




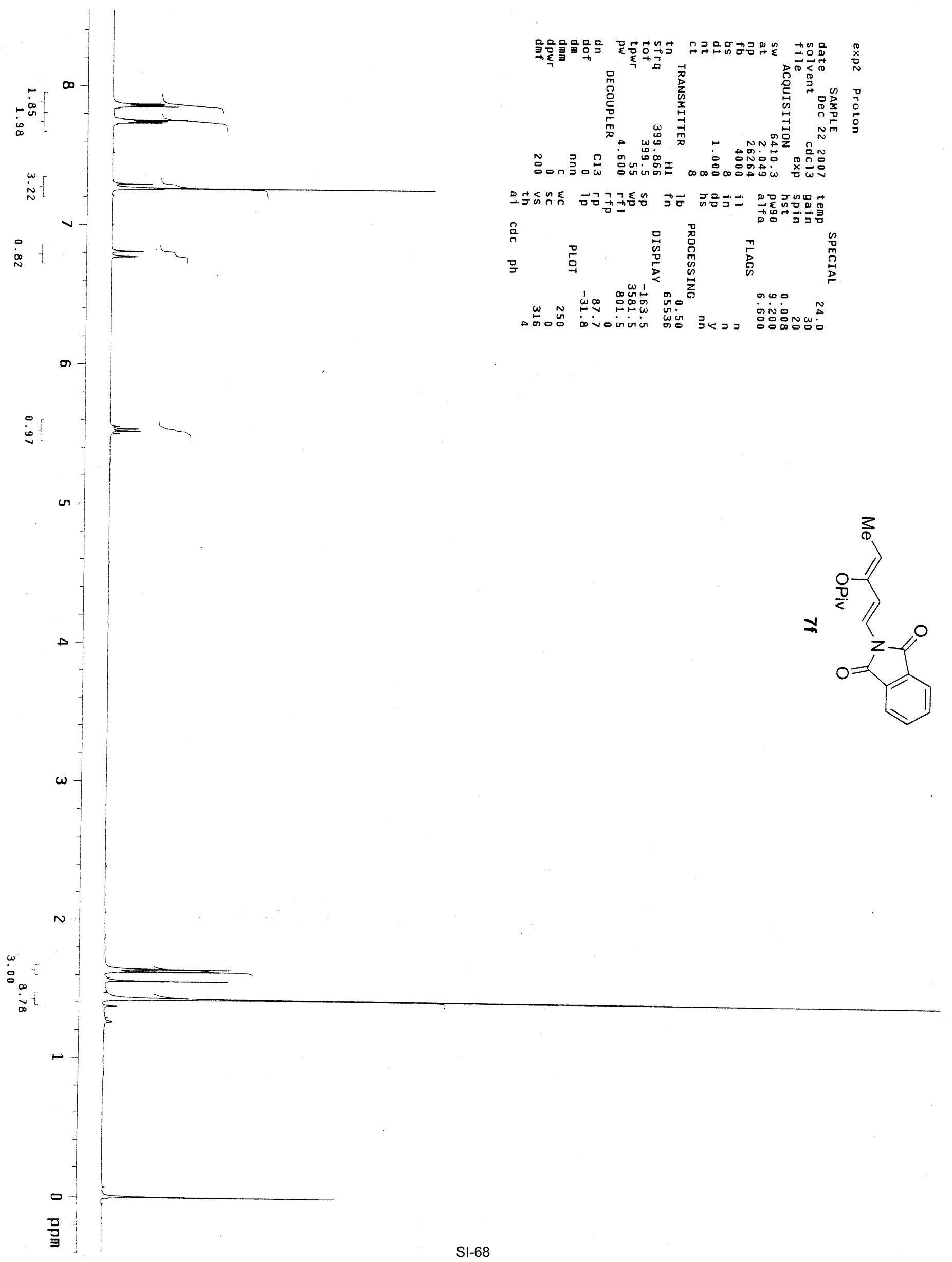




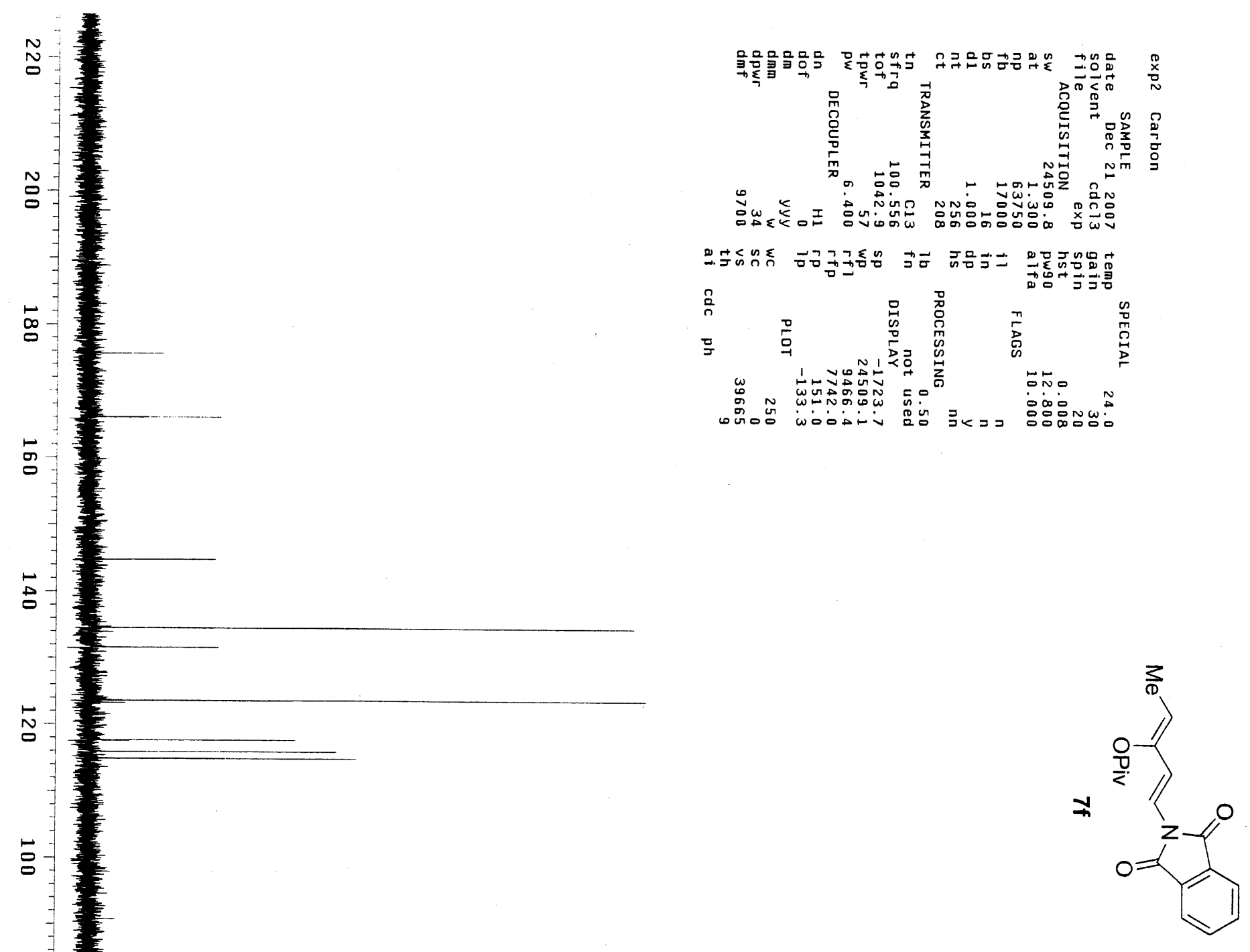




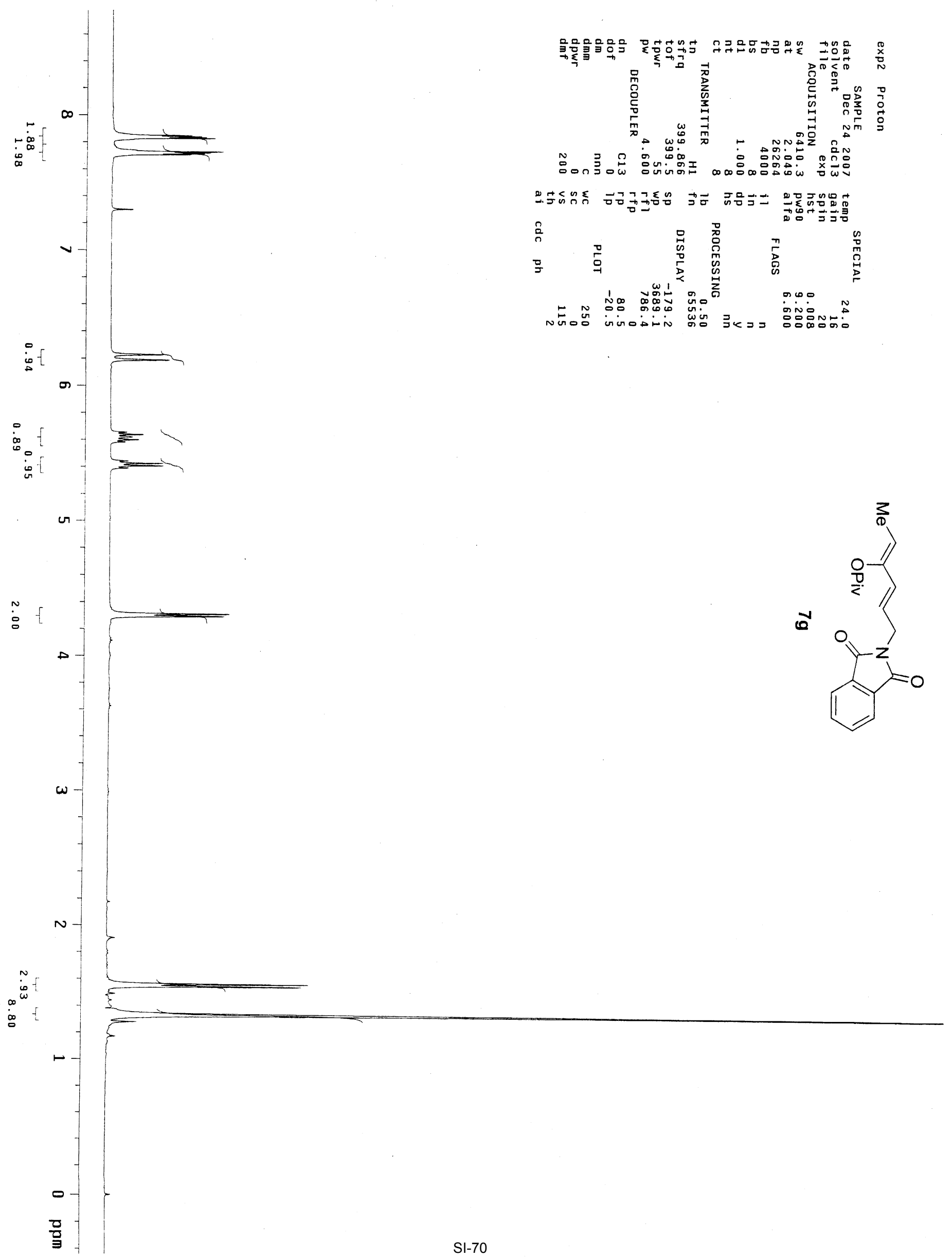




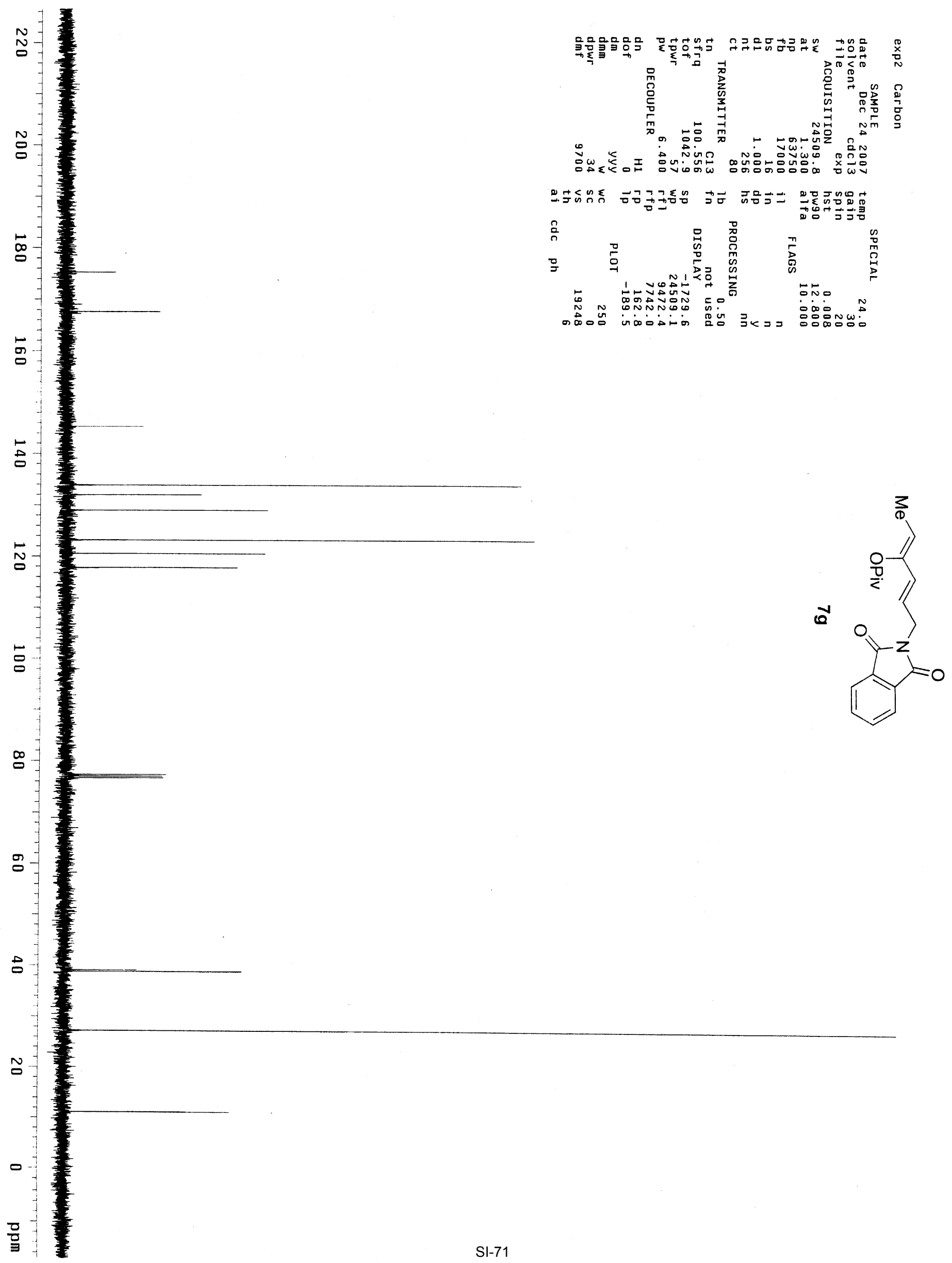




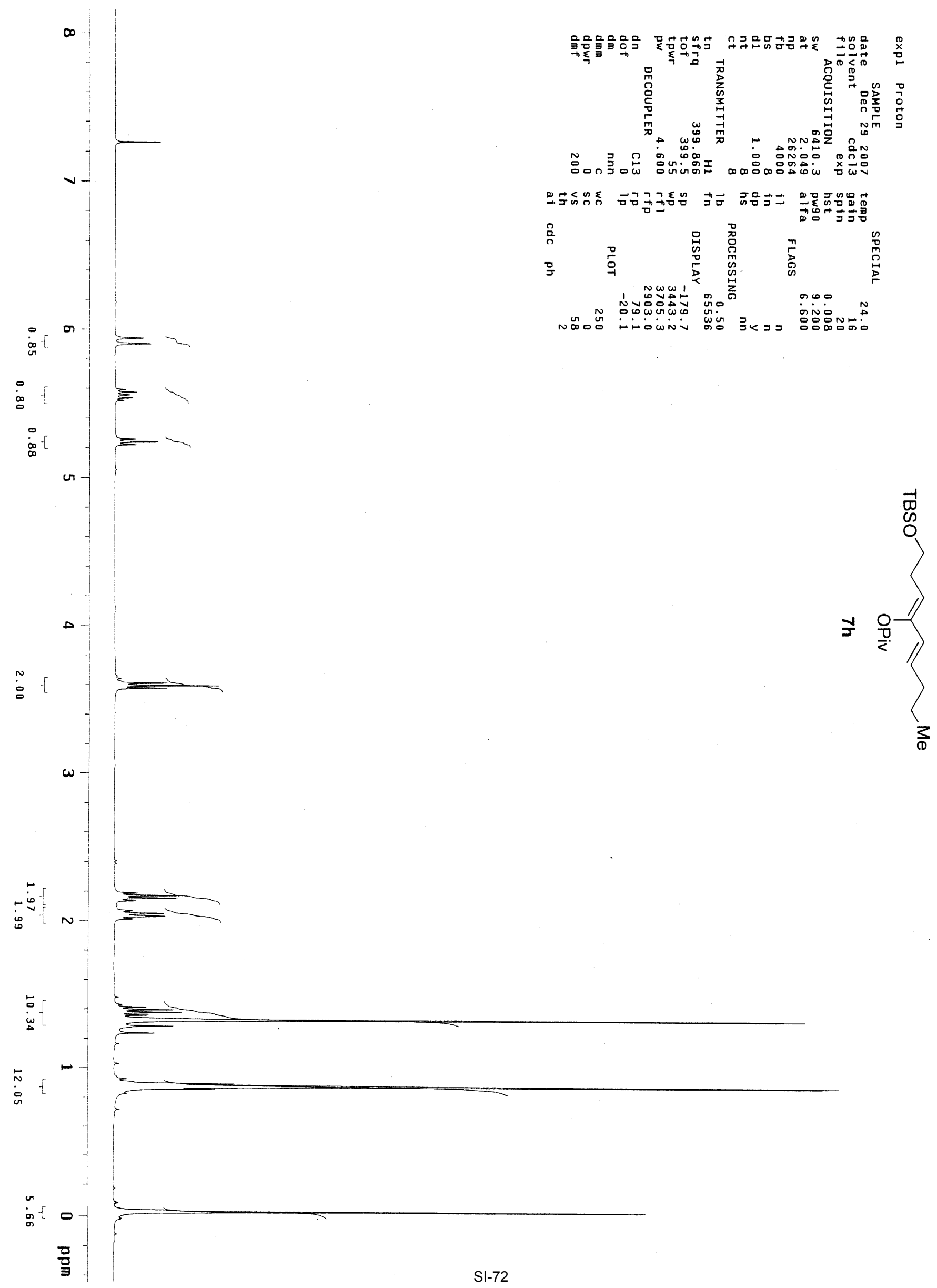



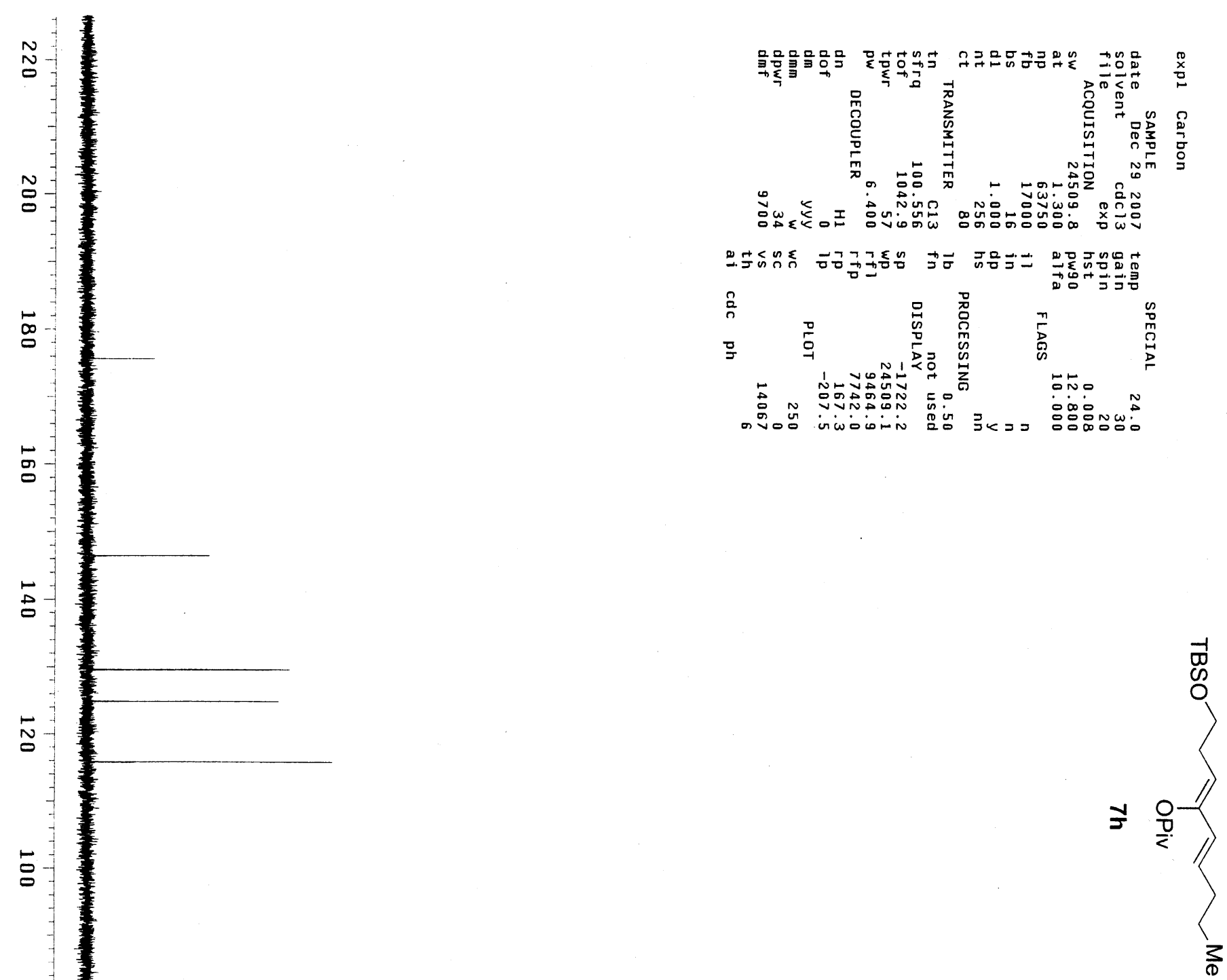


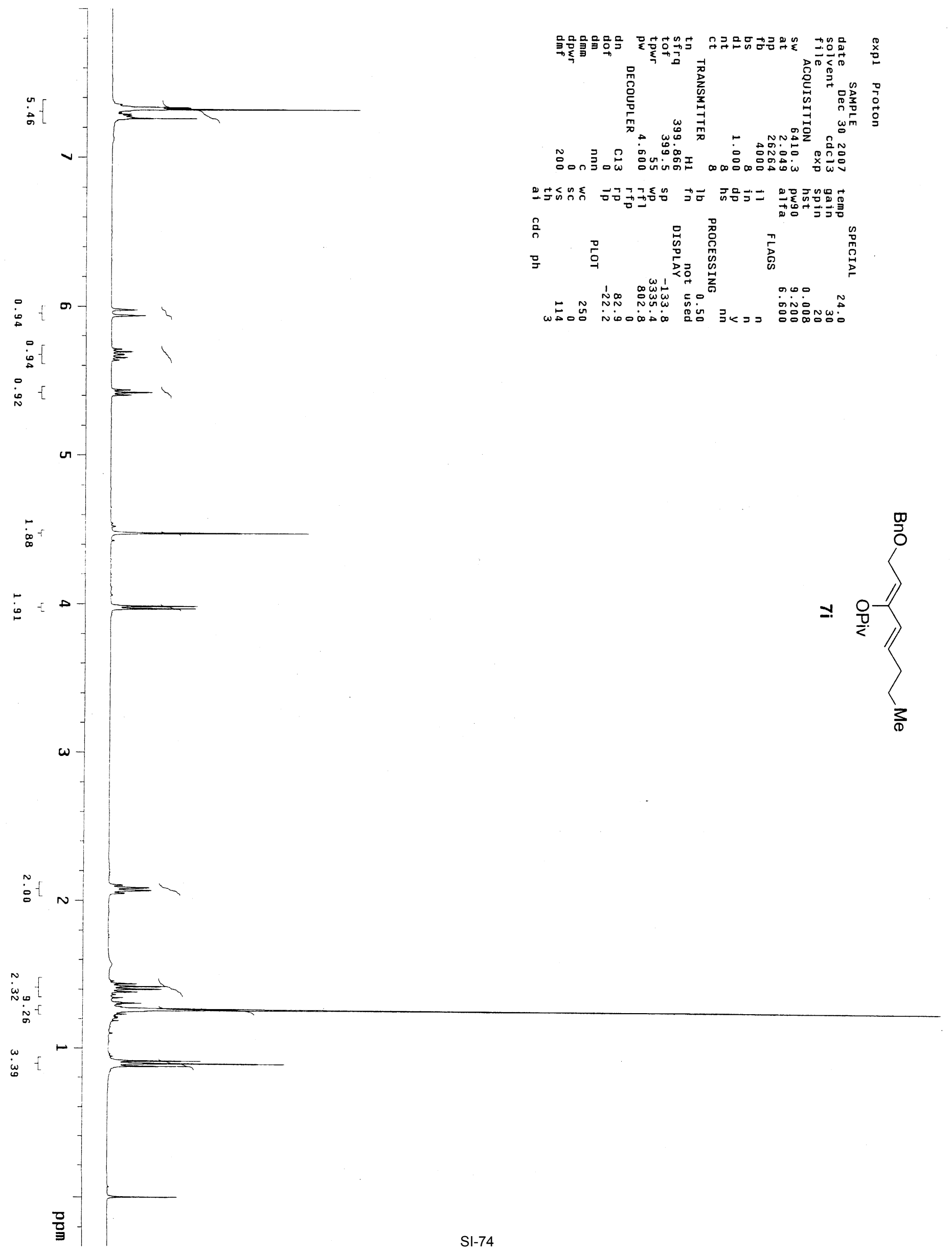




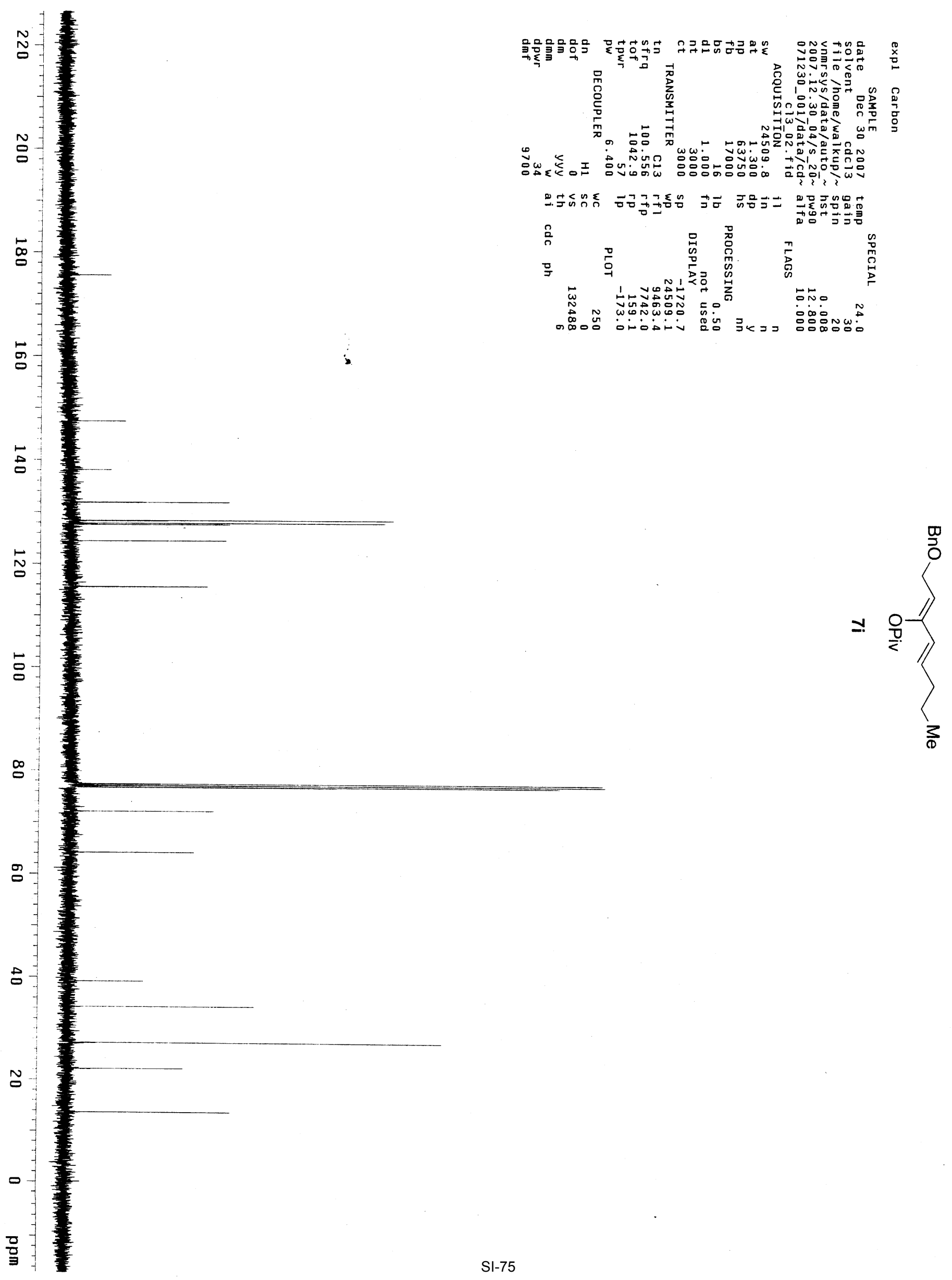



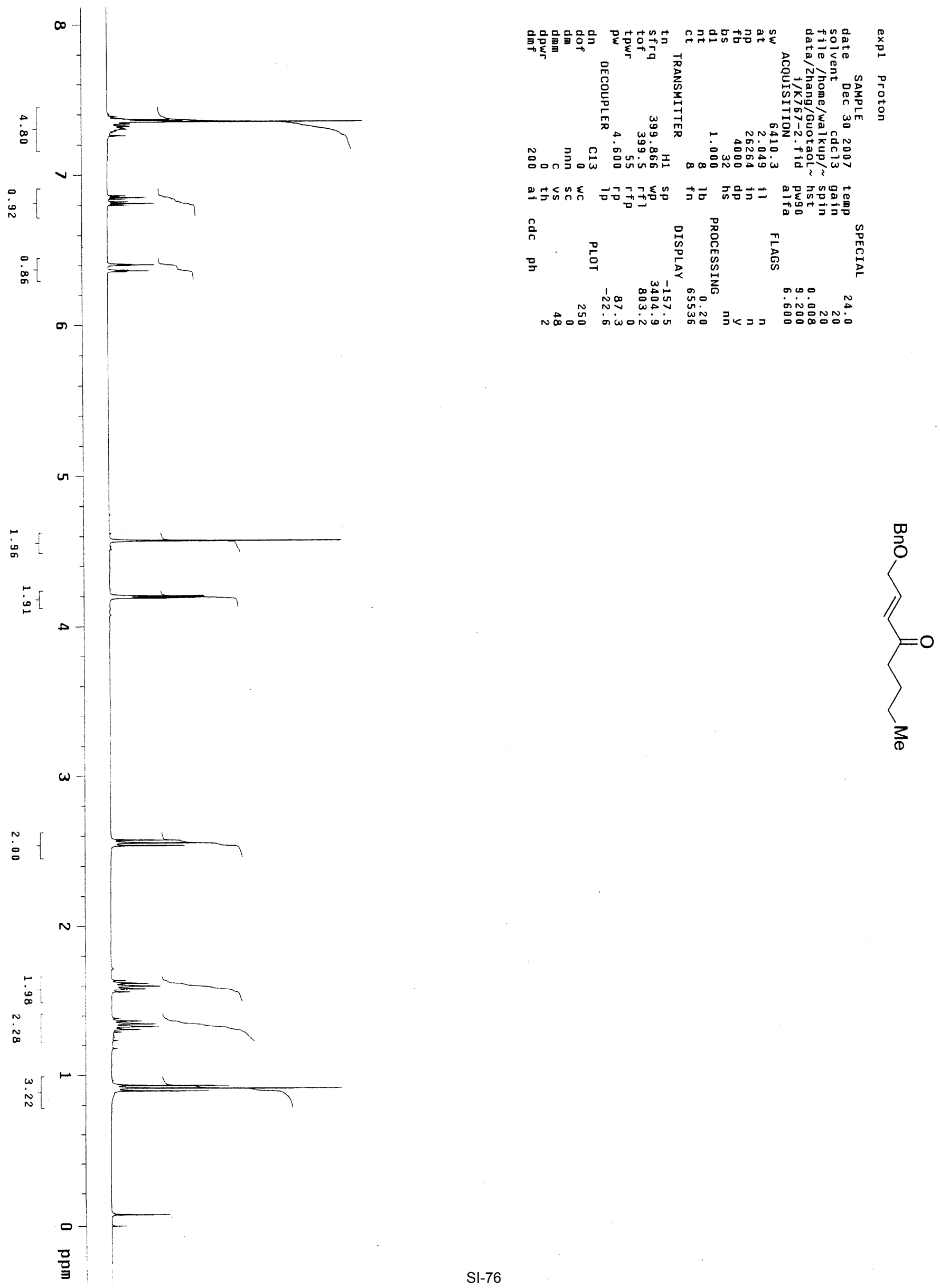

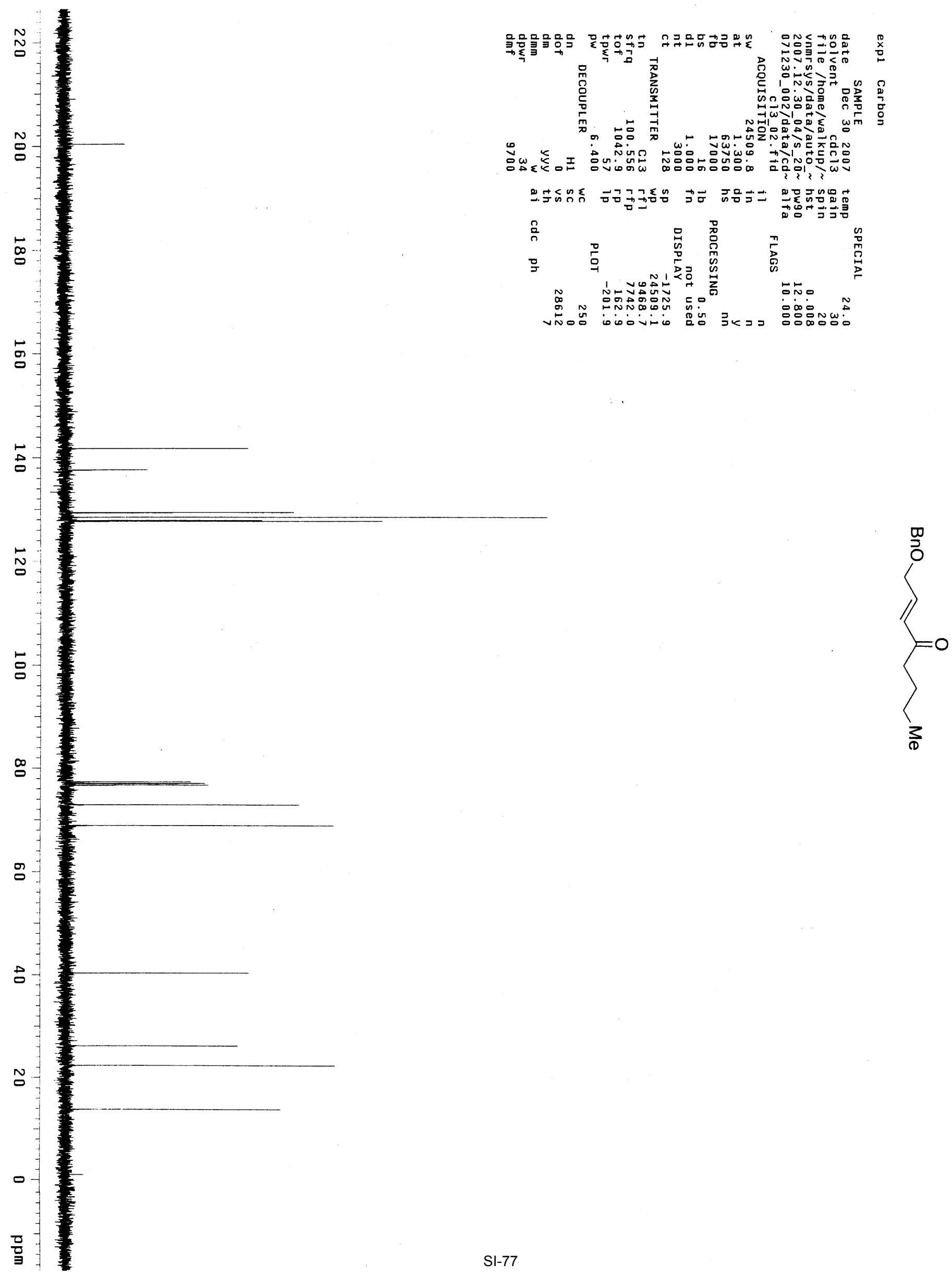


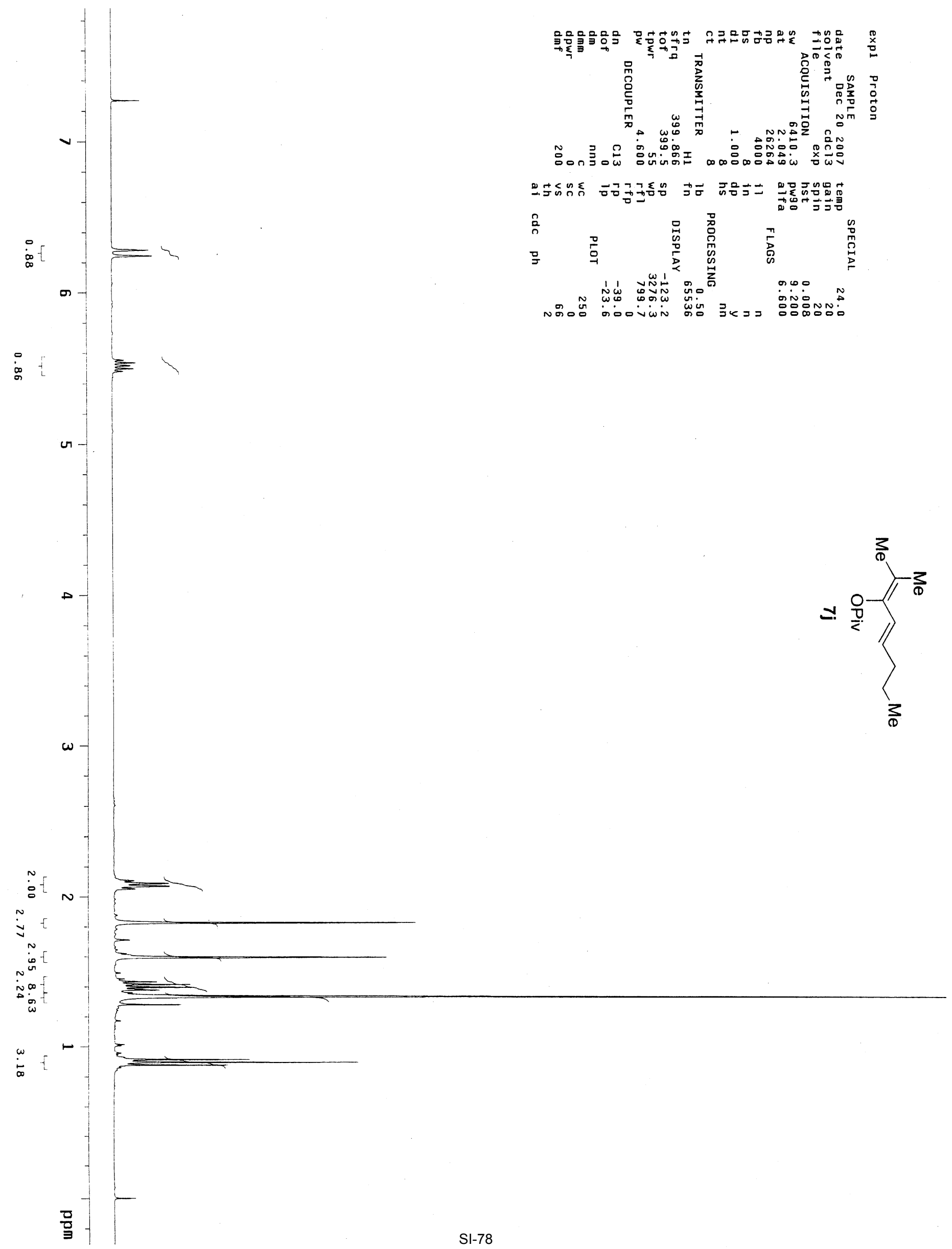




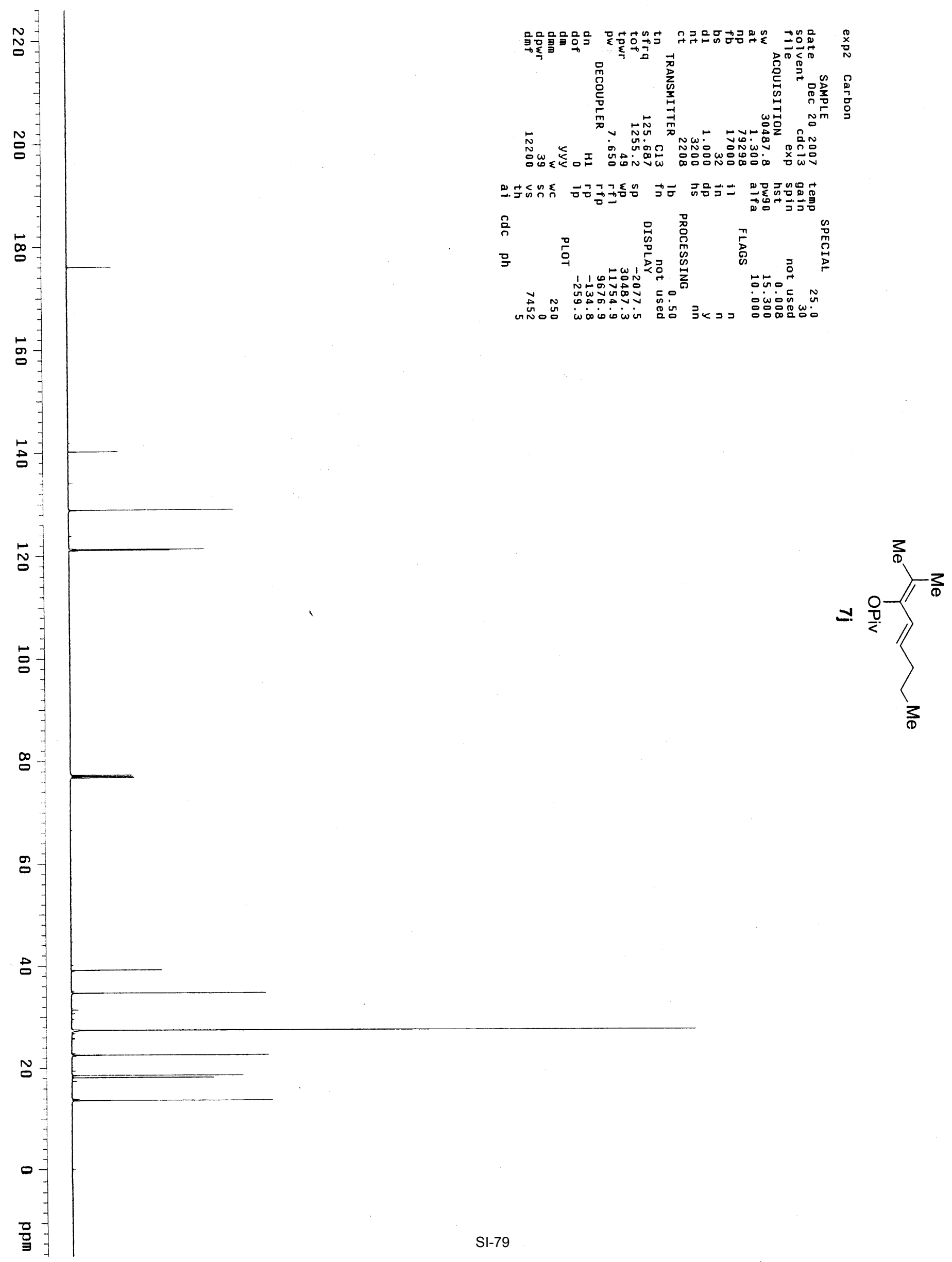



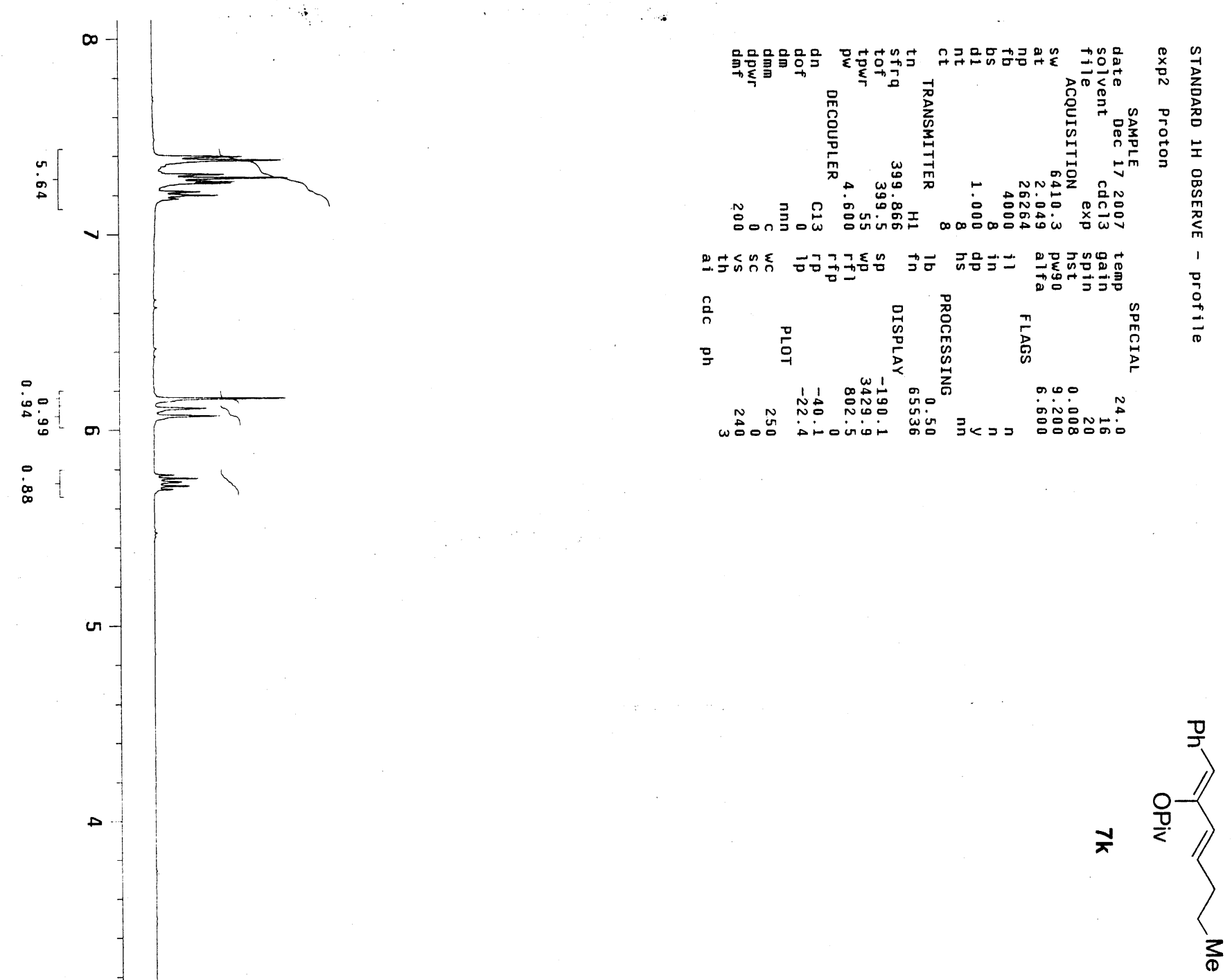

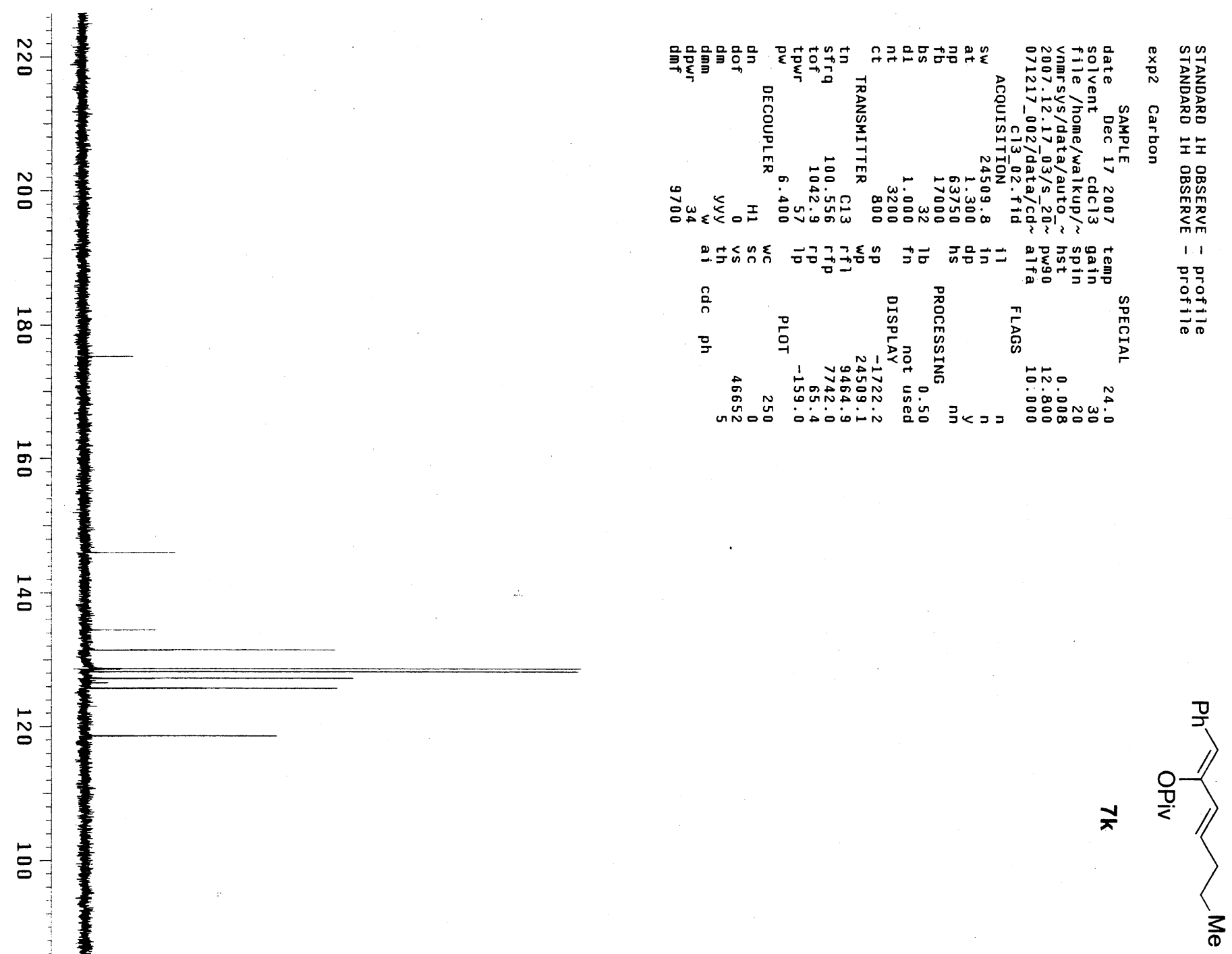

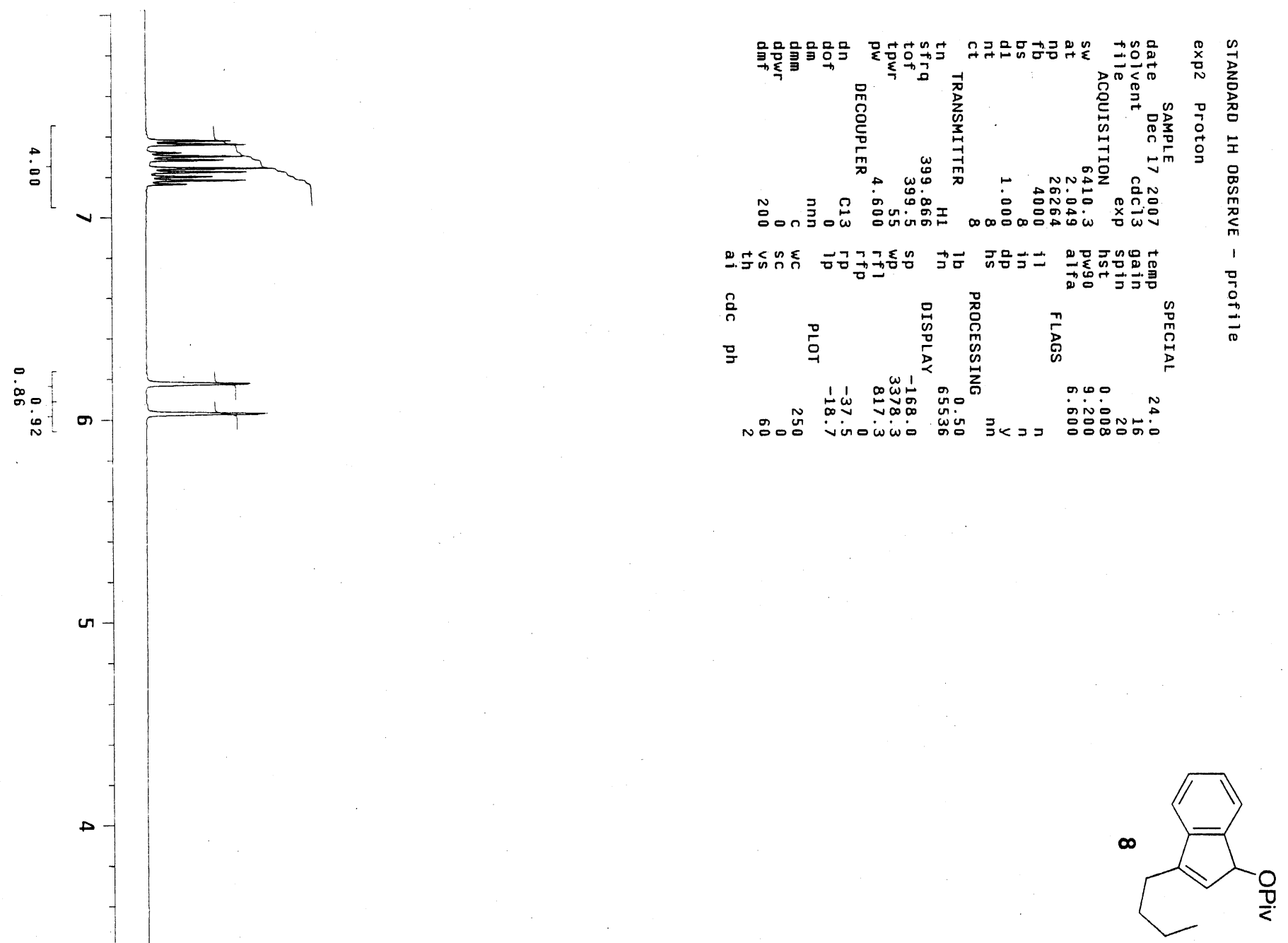

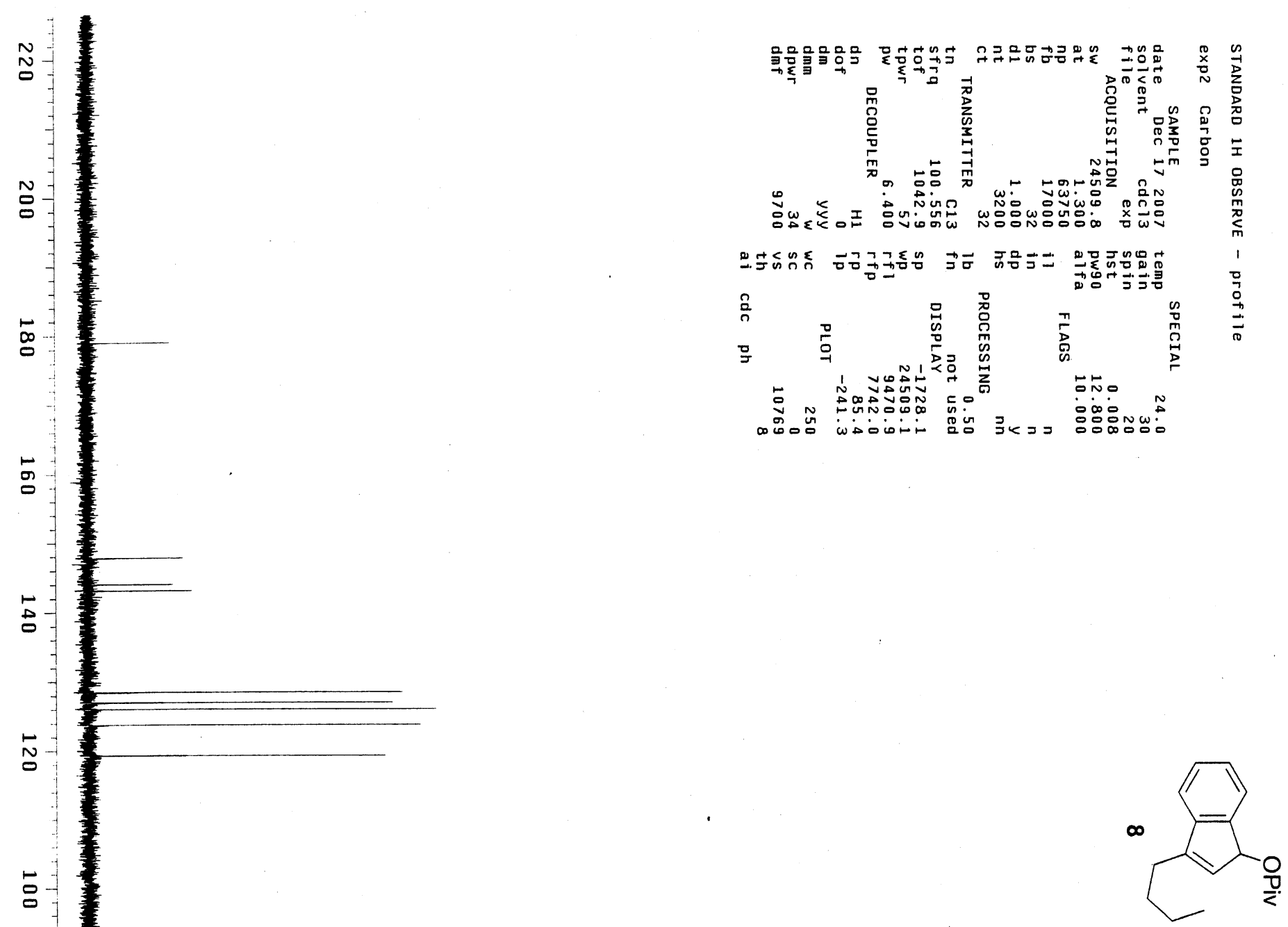


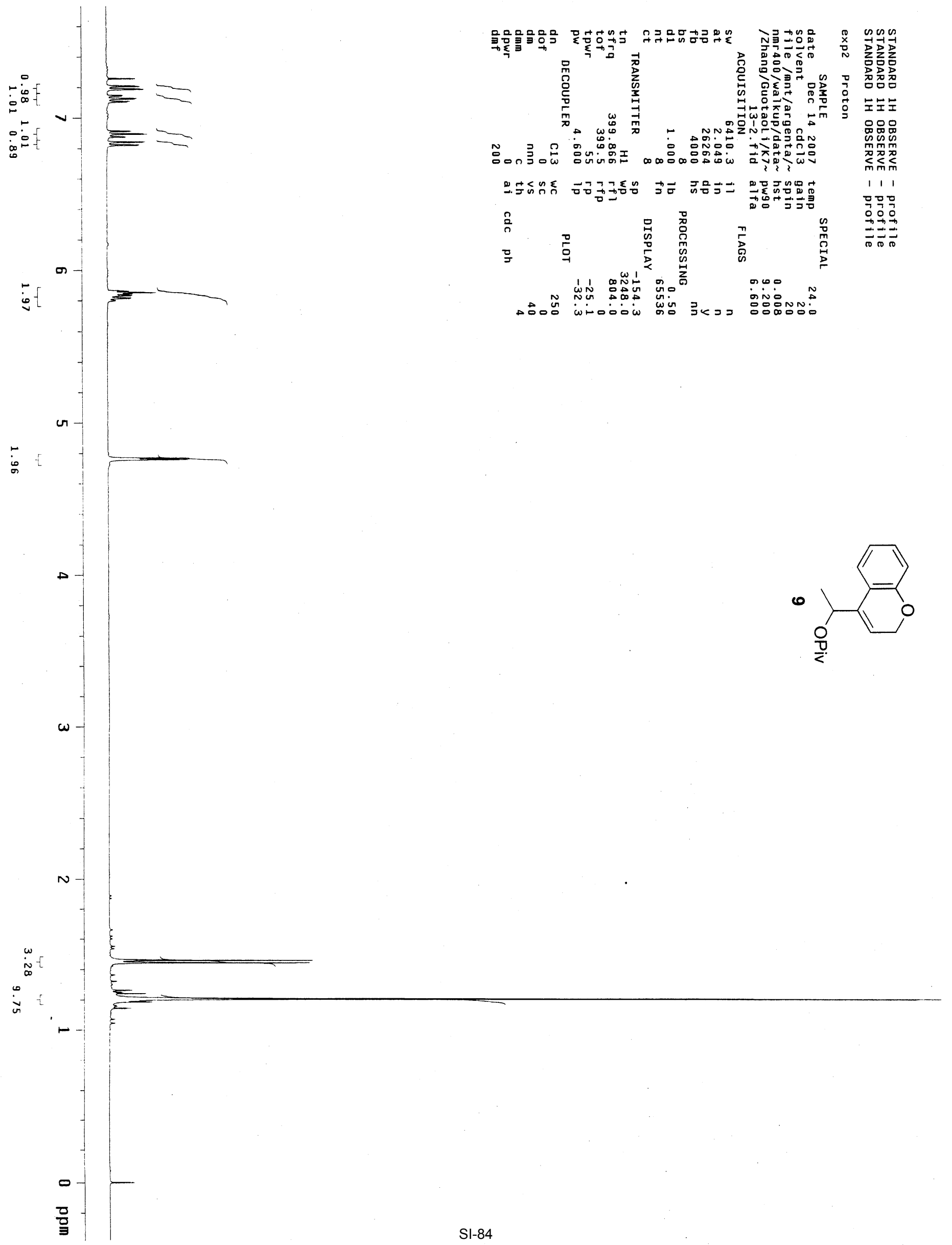




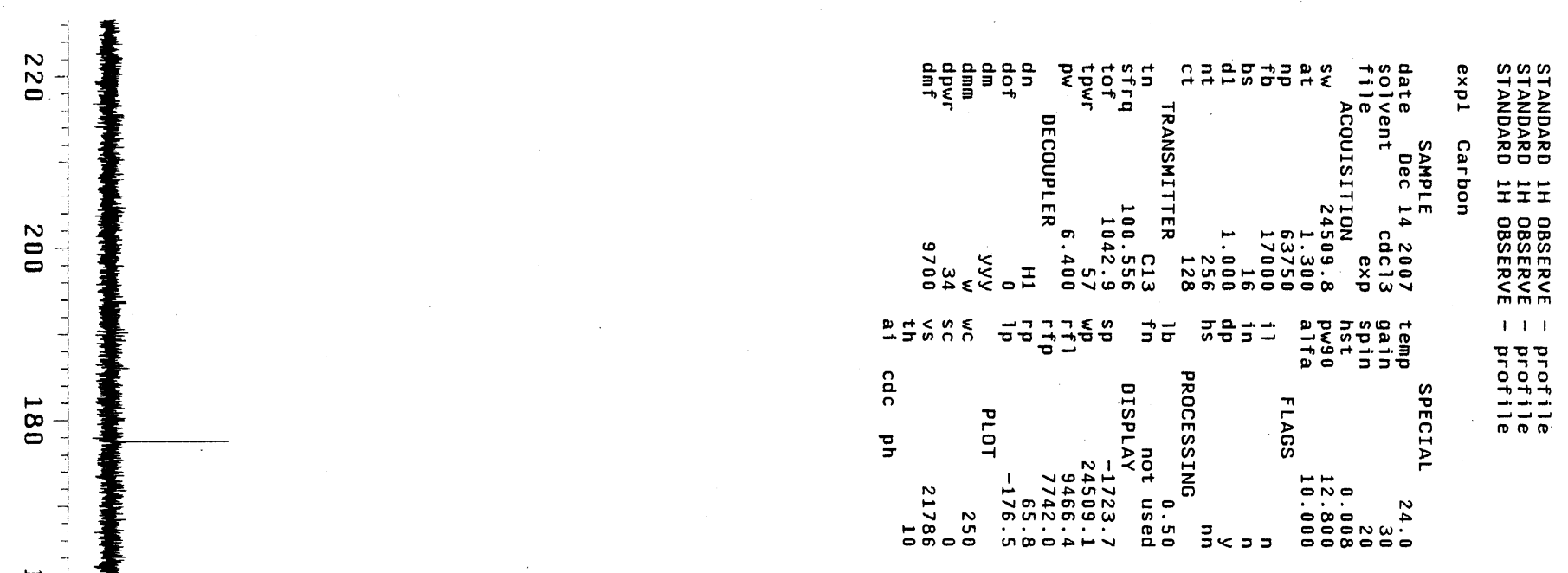

g

$\stackrel{\square}{\circ}$

N

뭉

$\stackrel{\infty}{\circ}$

g

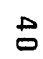

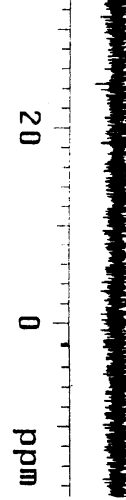

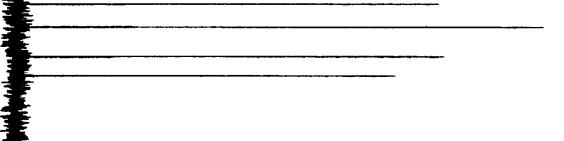

.

$\stackrel{\infty}{\circ}$

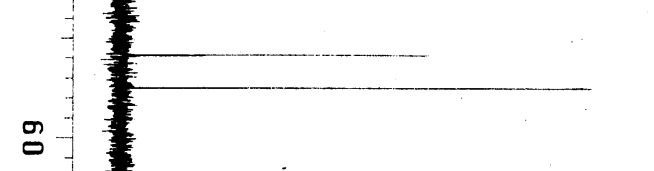

$D$

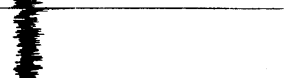

0<smiles>CC(O)C1=CCOc2ccccc21</smiles> 


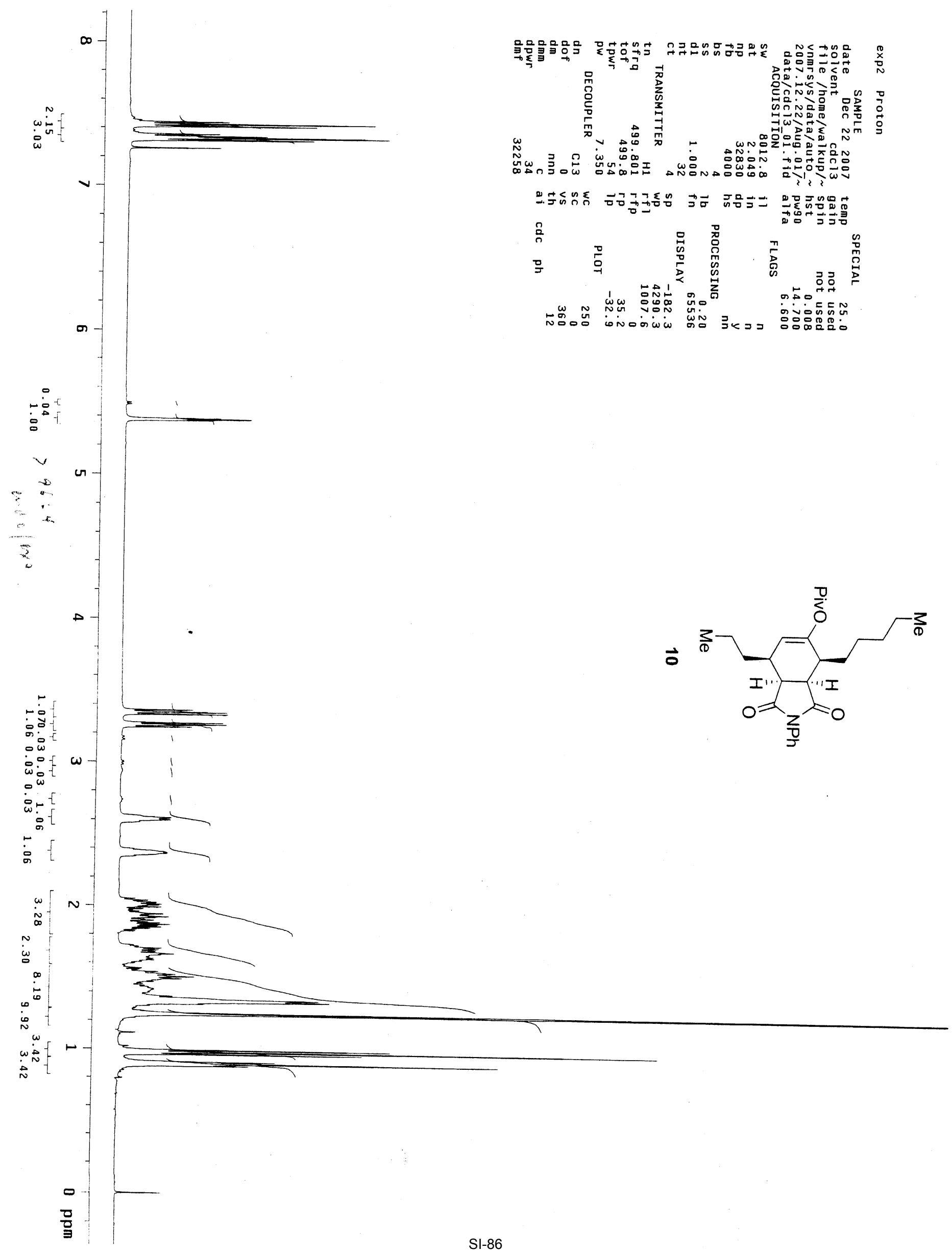




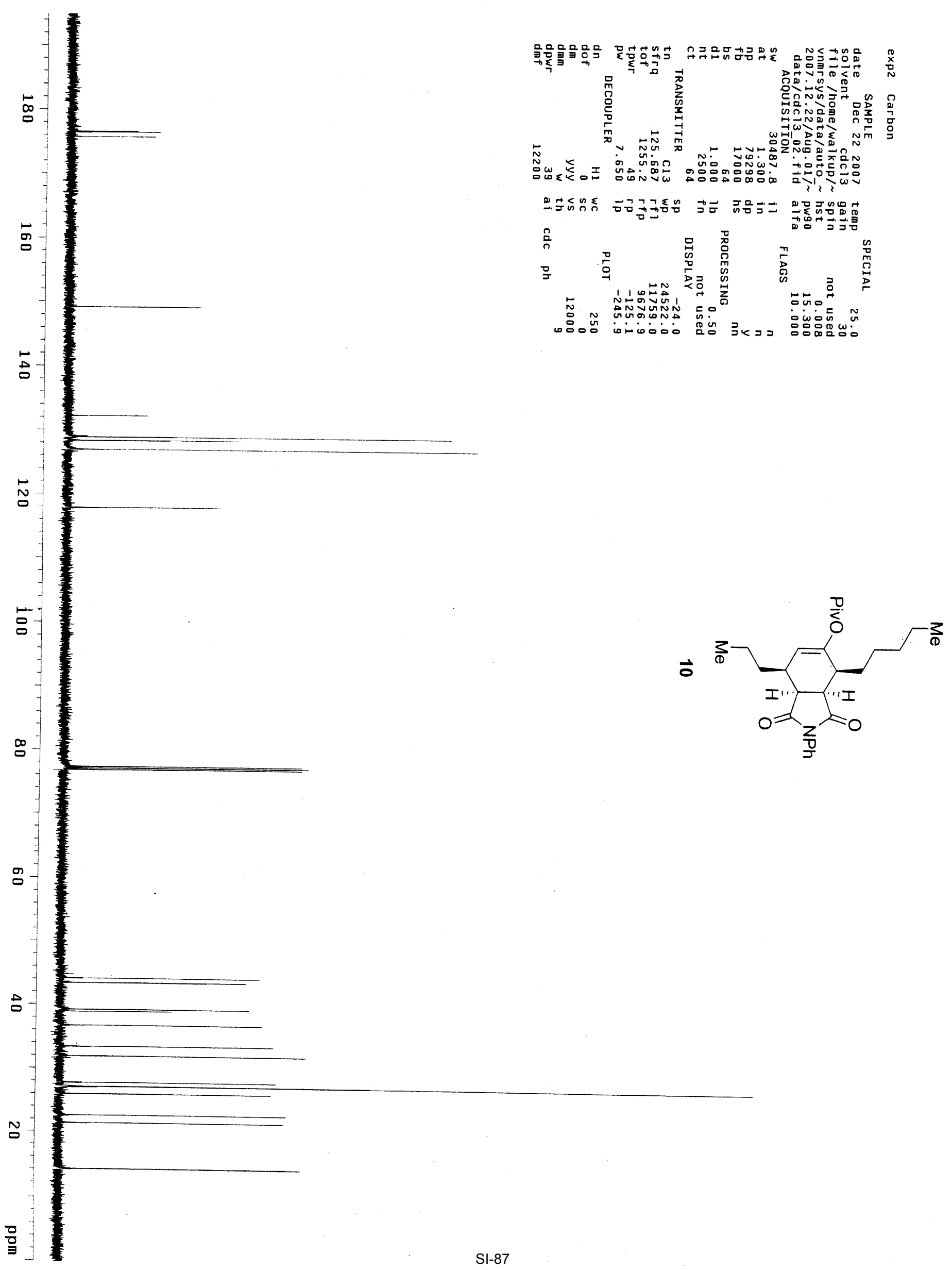




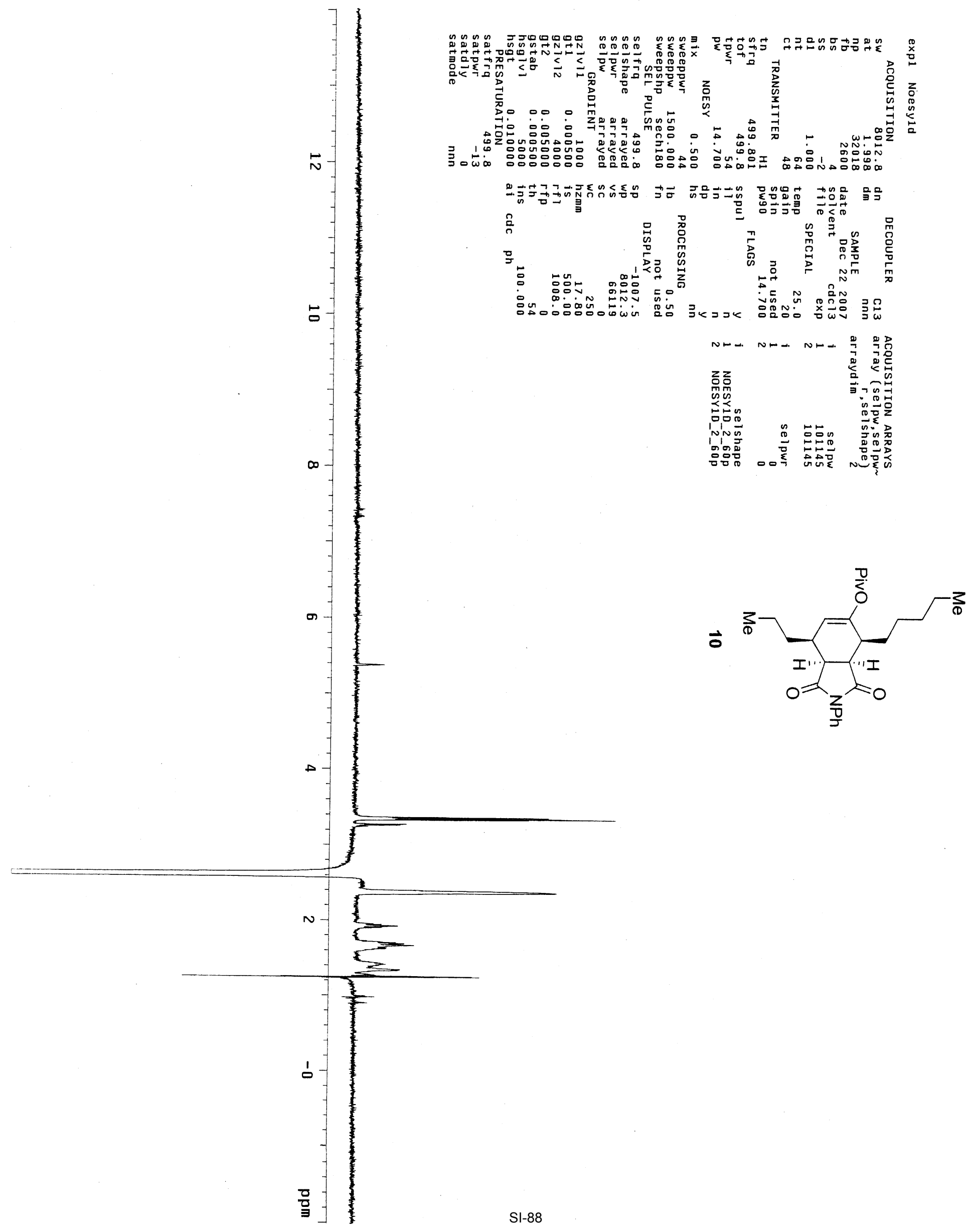




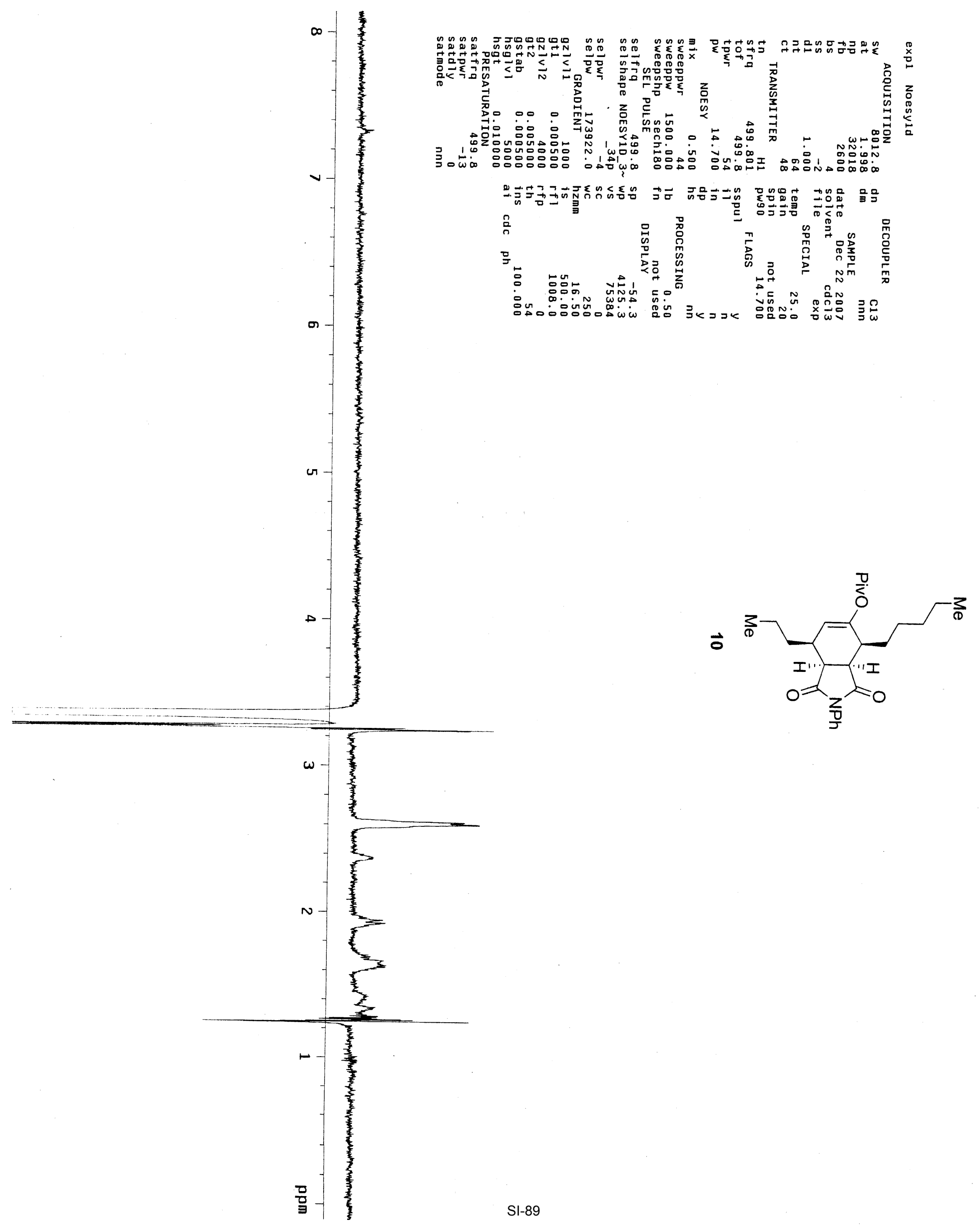




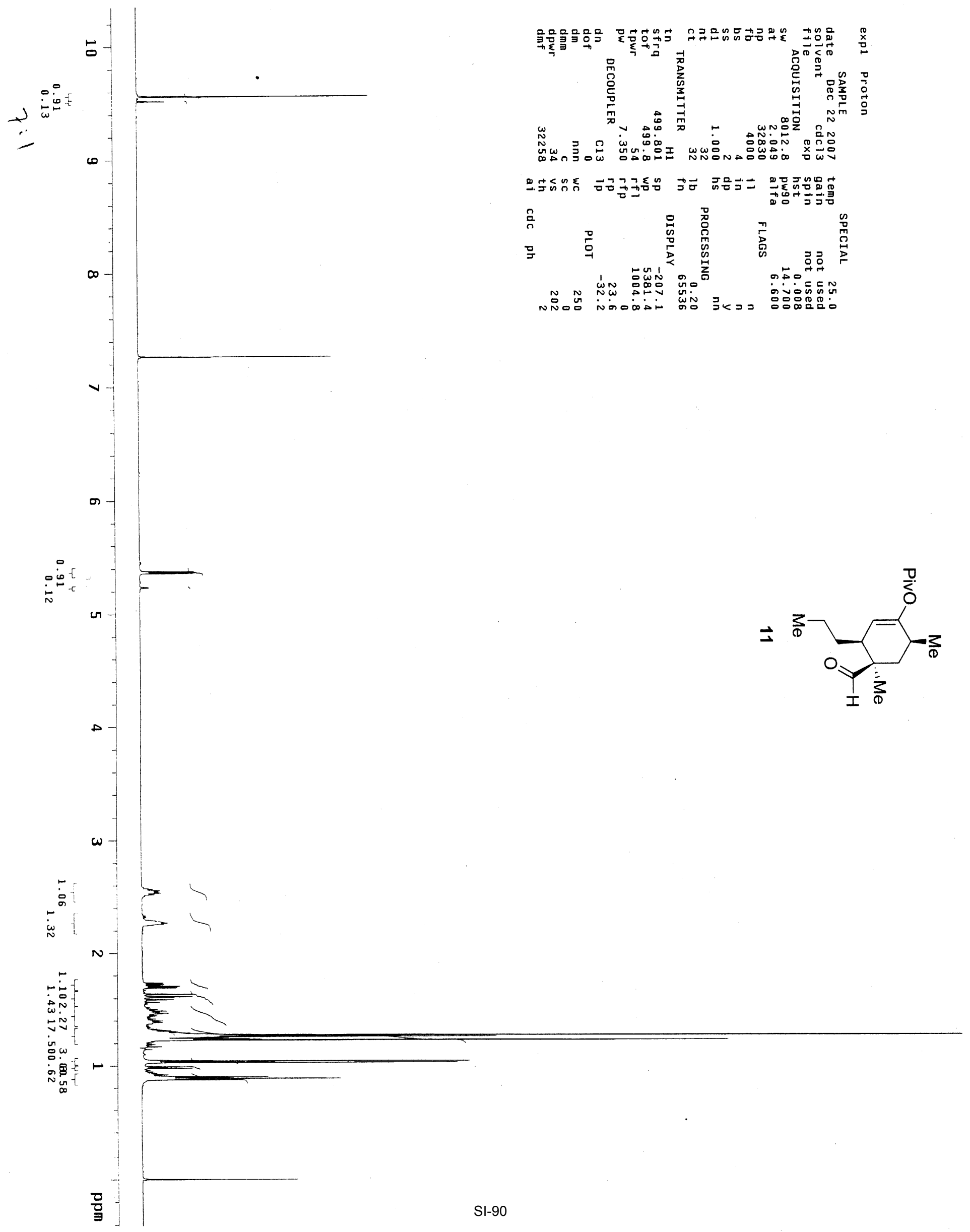




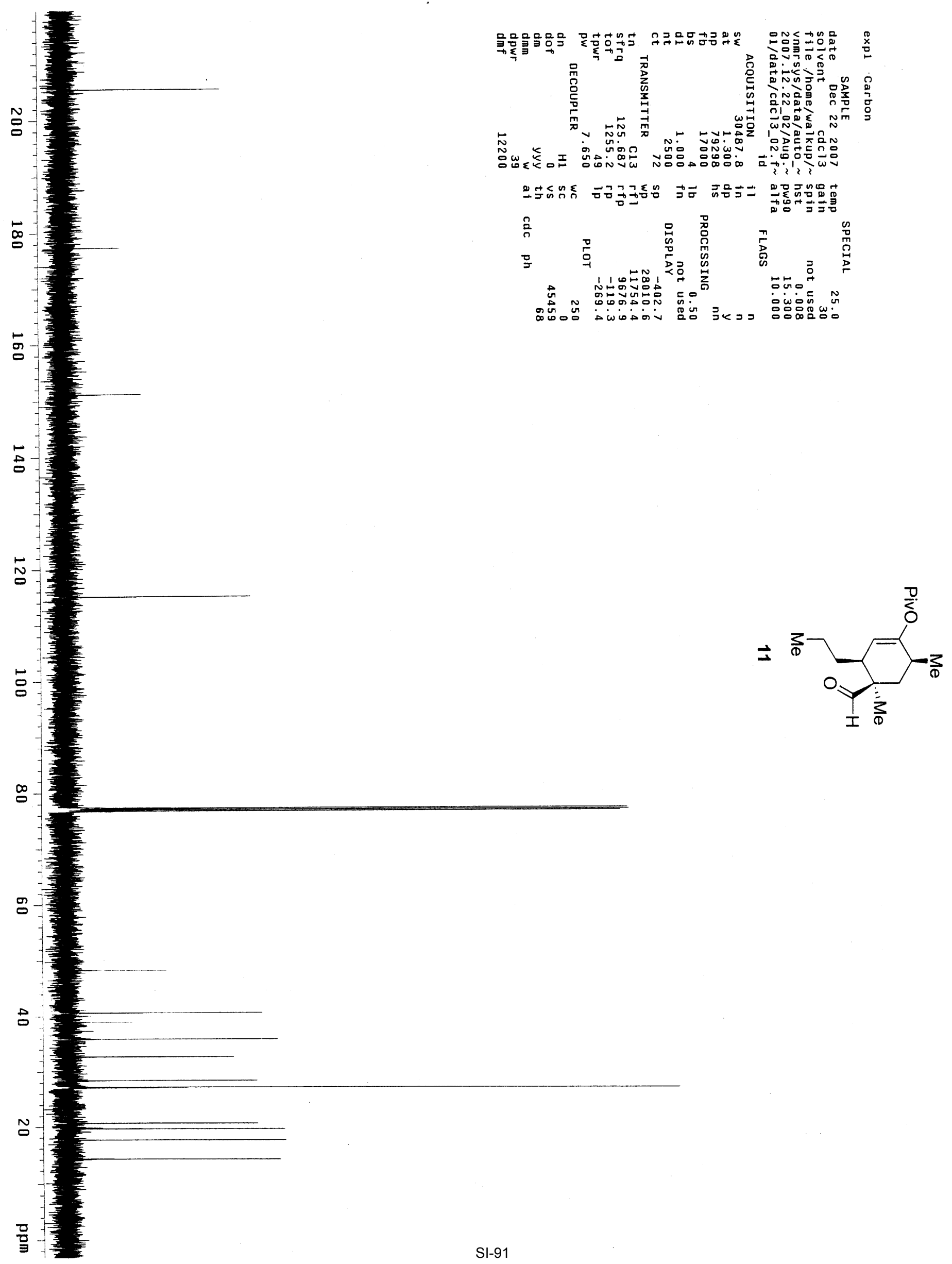




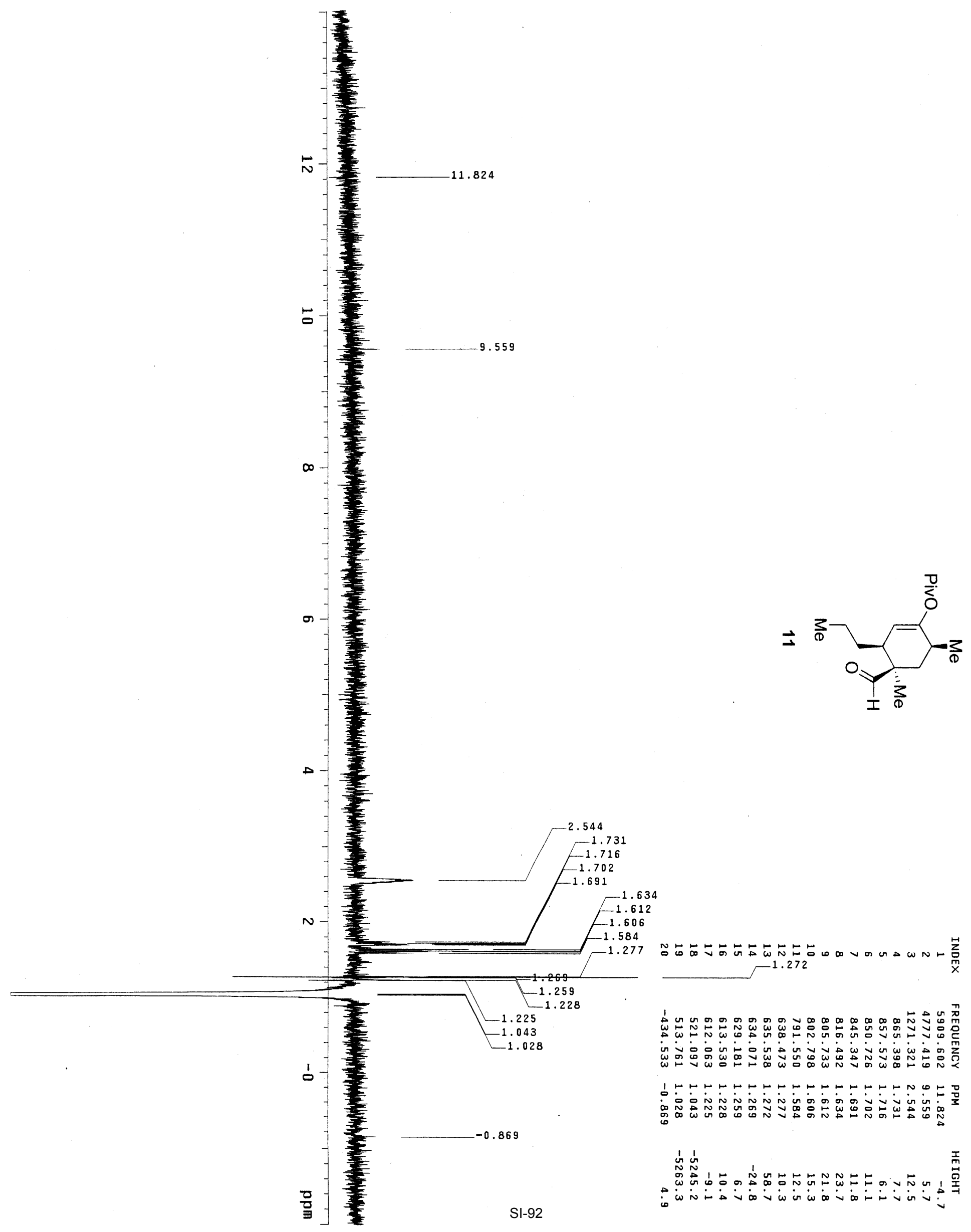




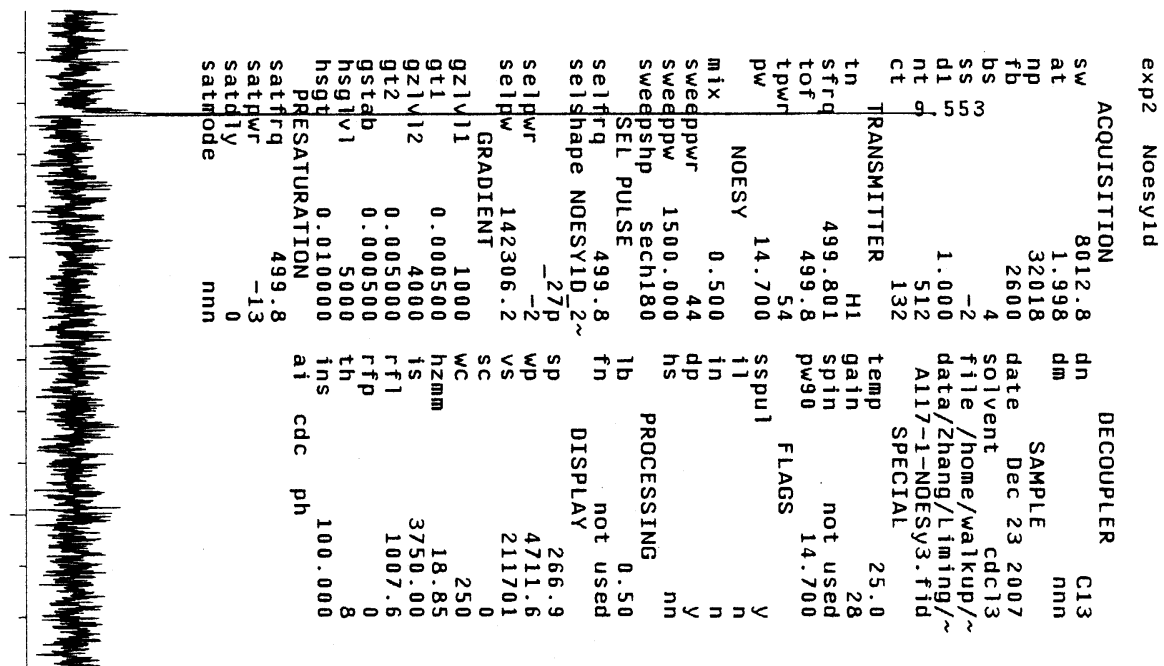

a

$\omega$

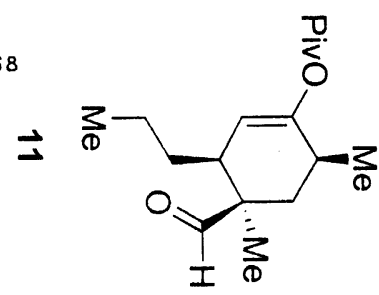

$\triangle$

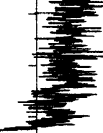

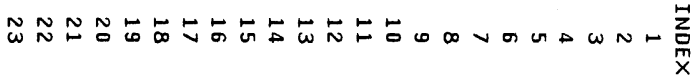

$N$

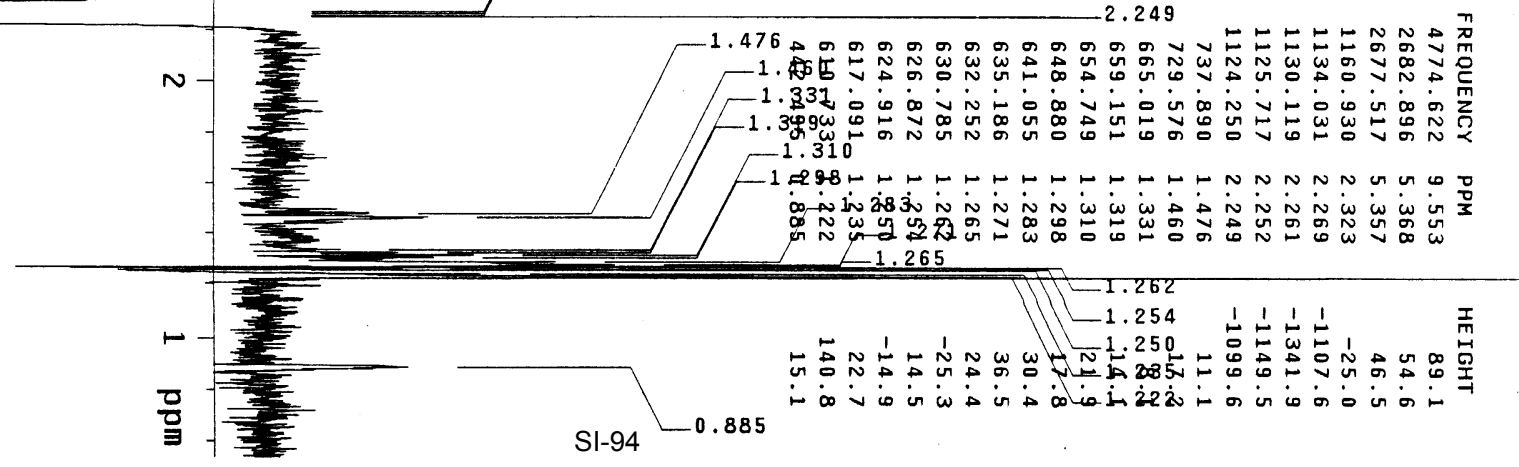




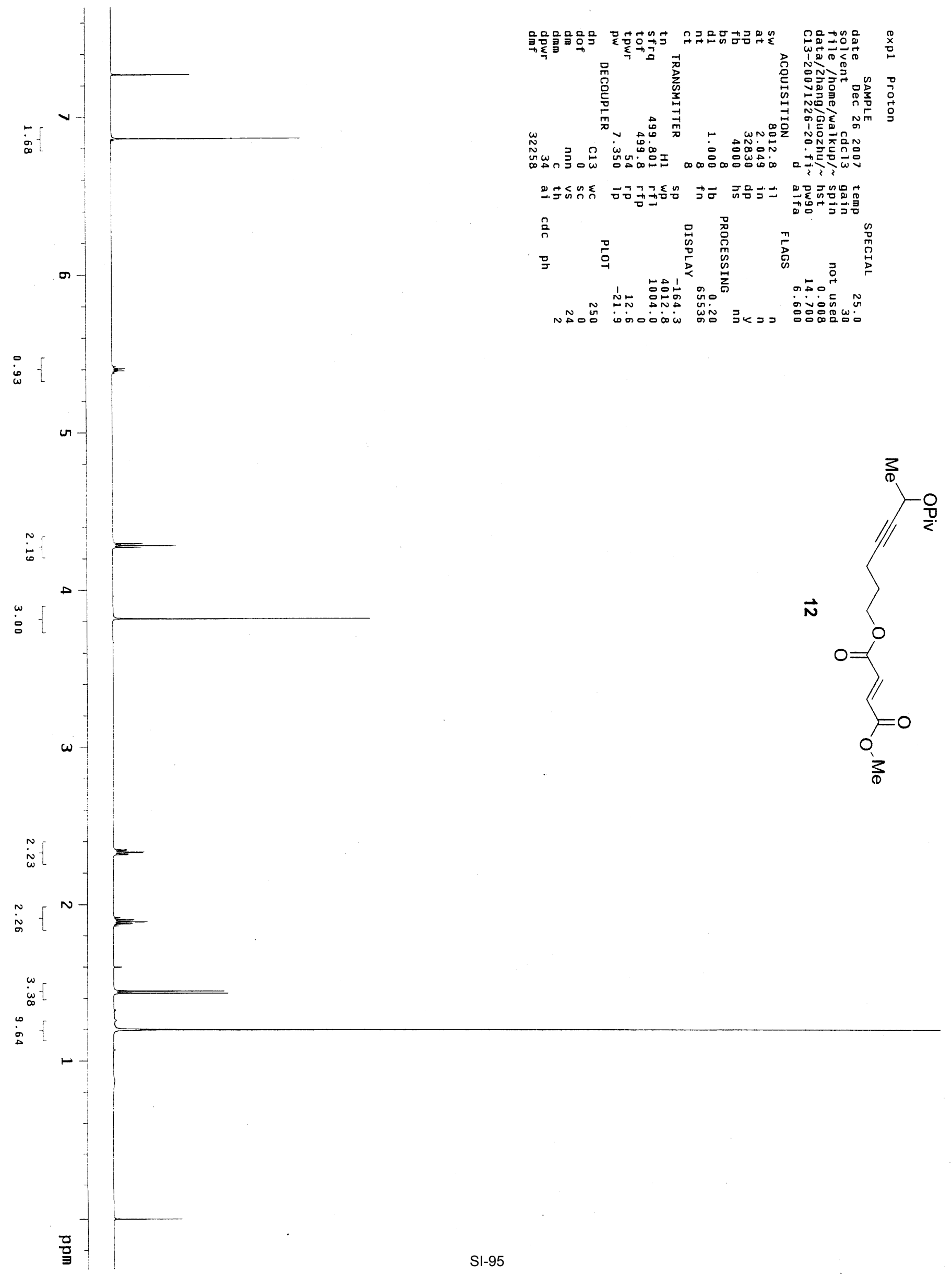



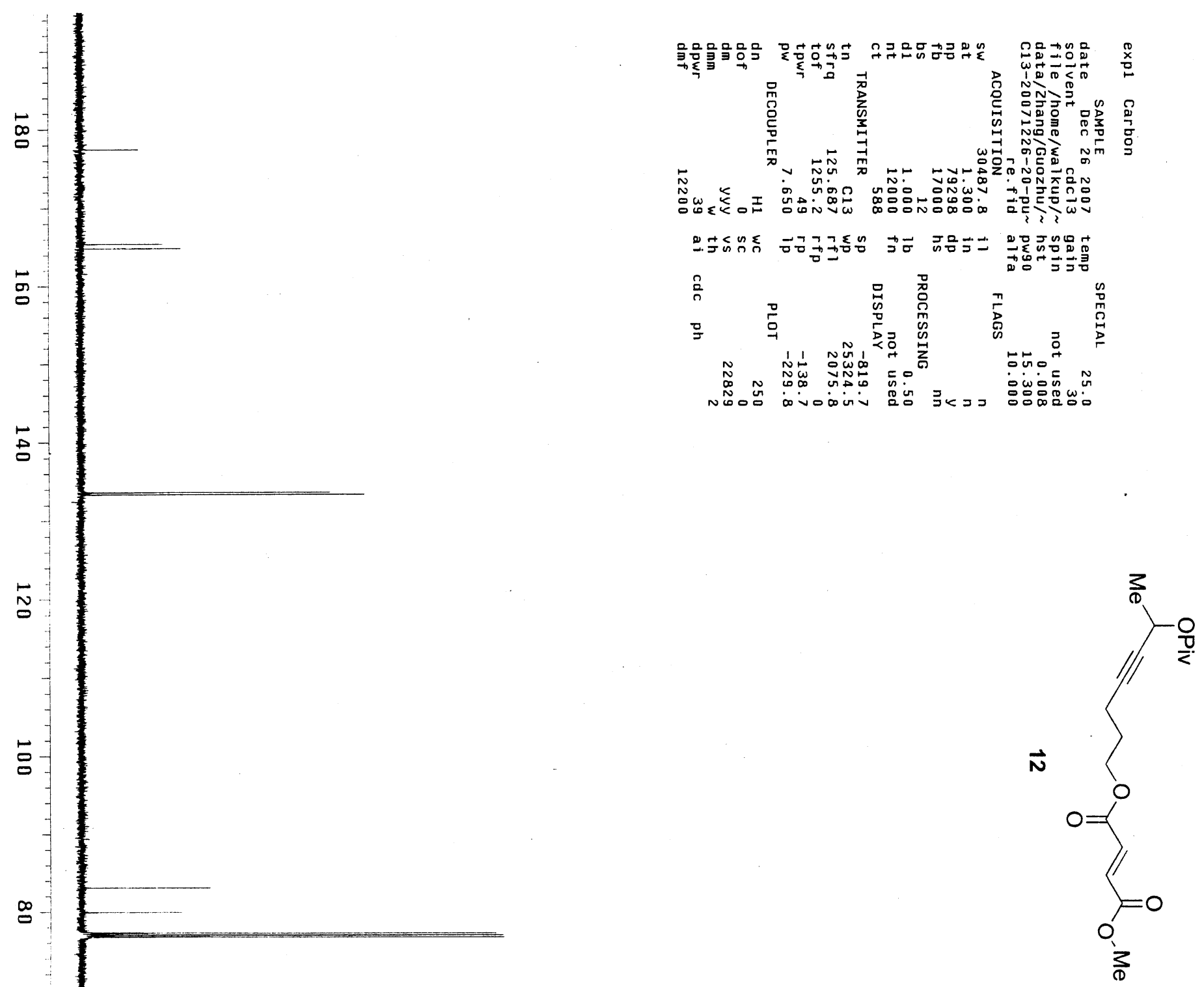


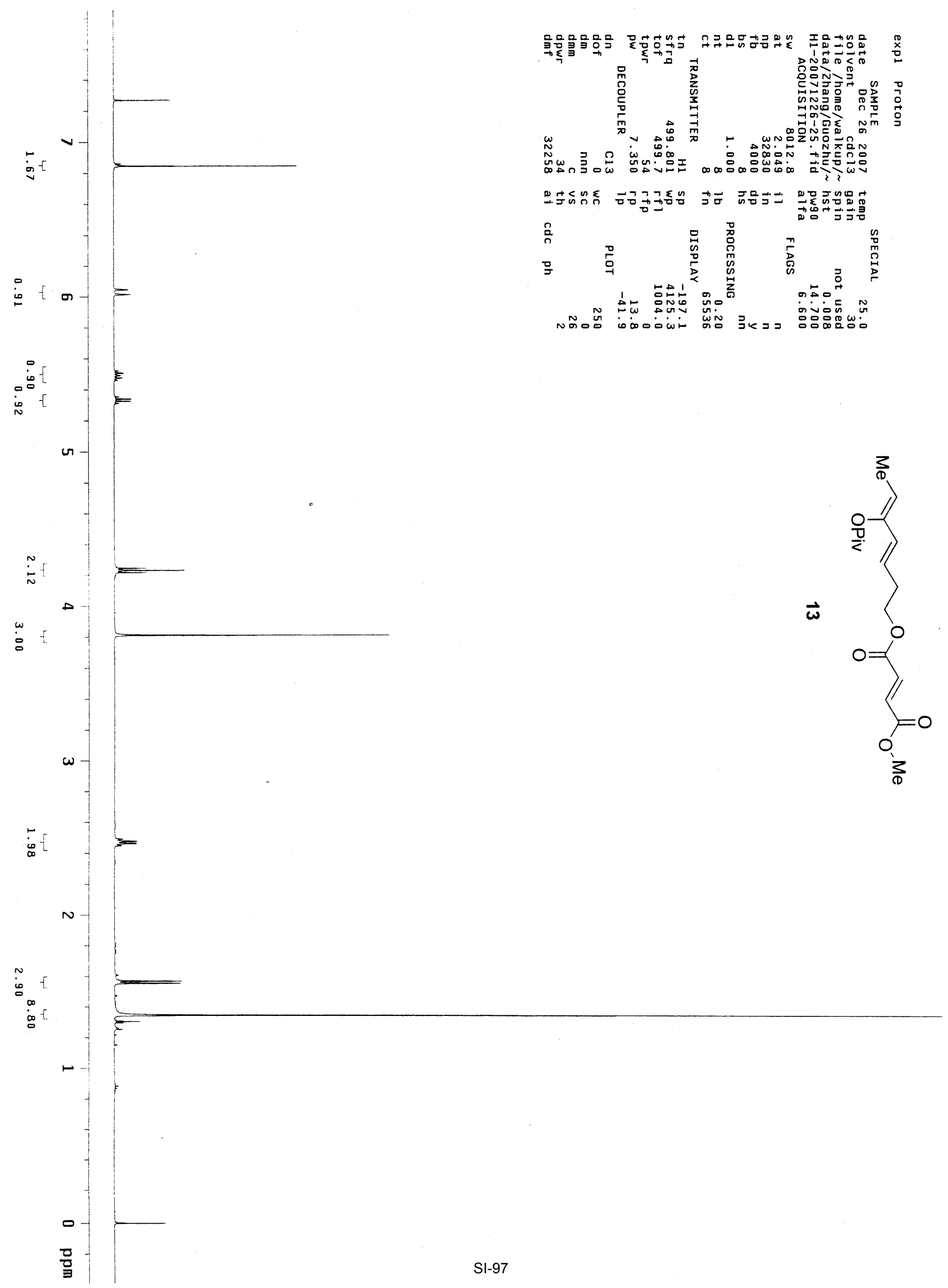




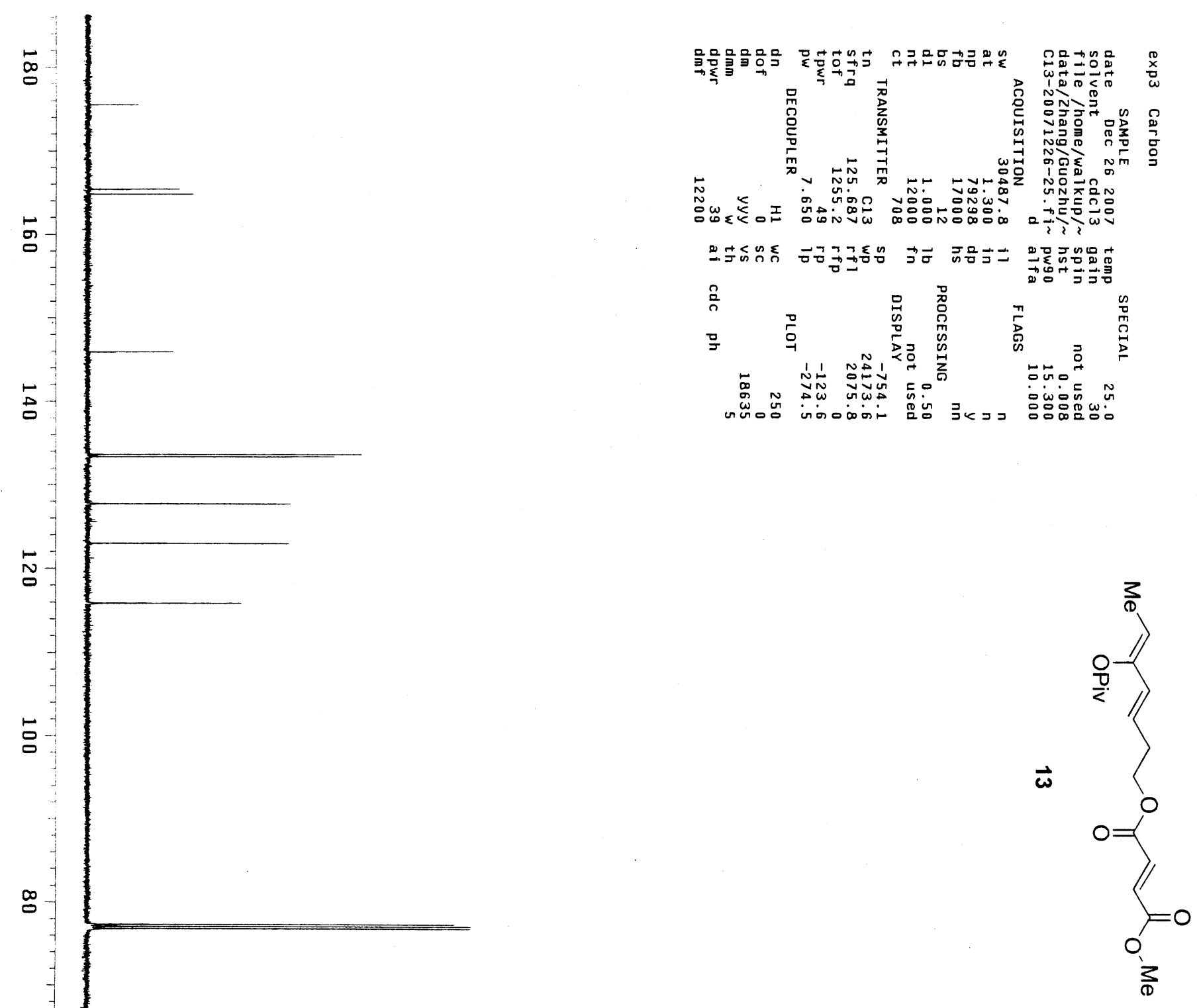

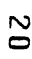

밀 


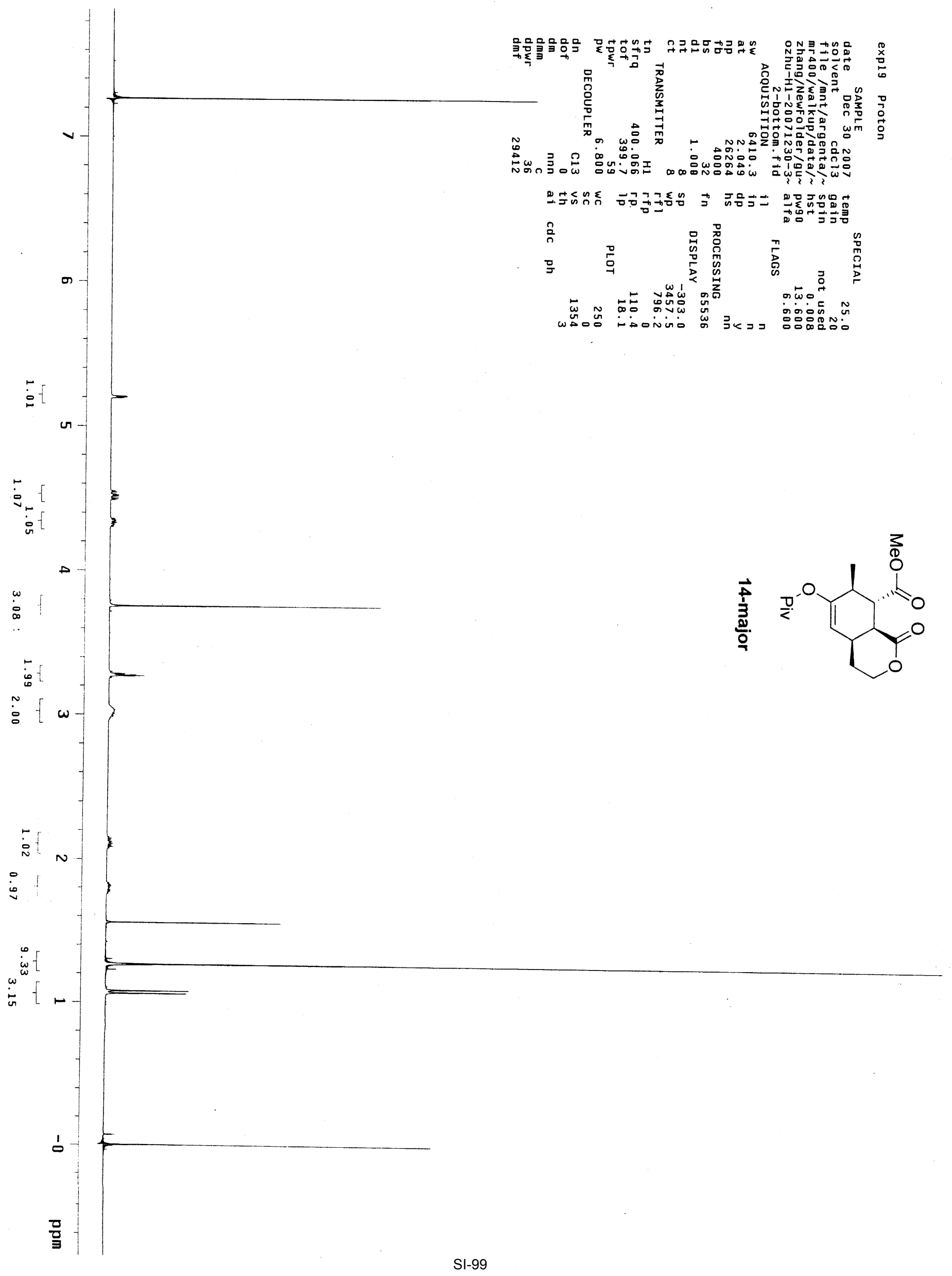



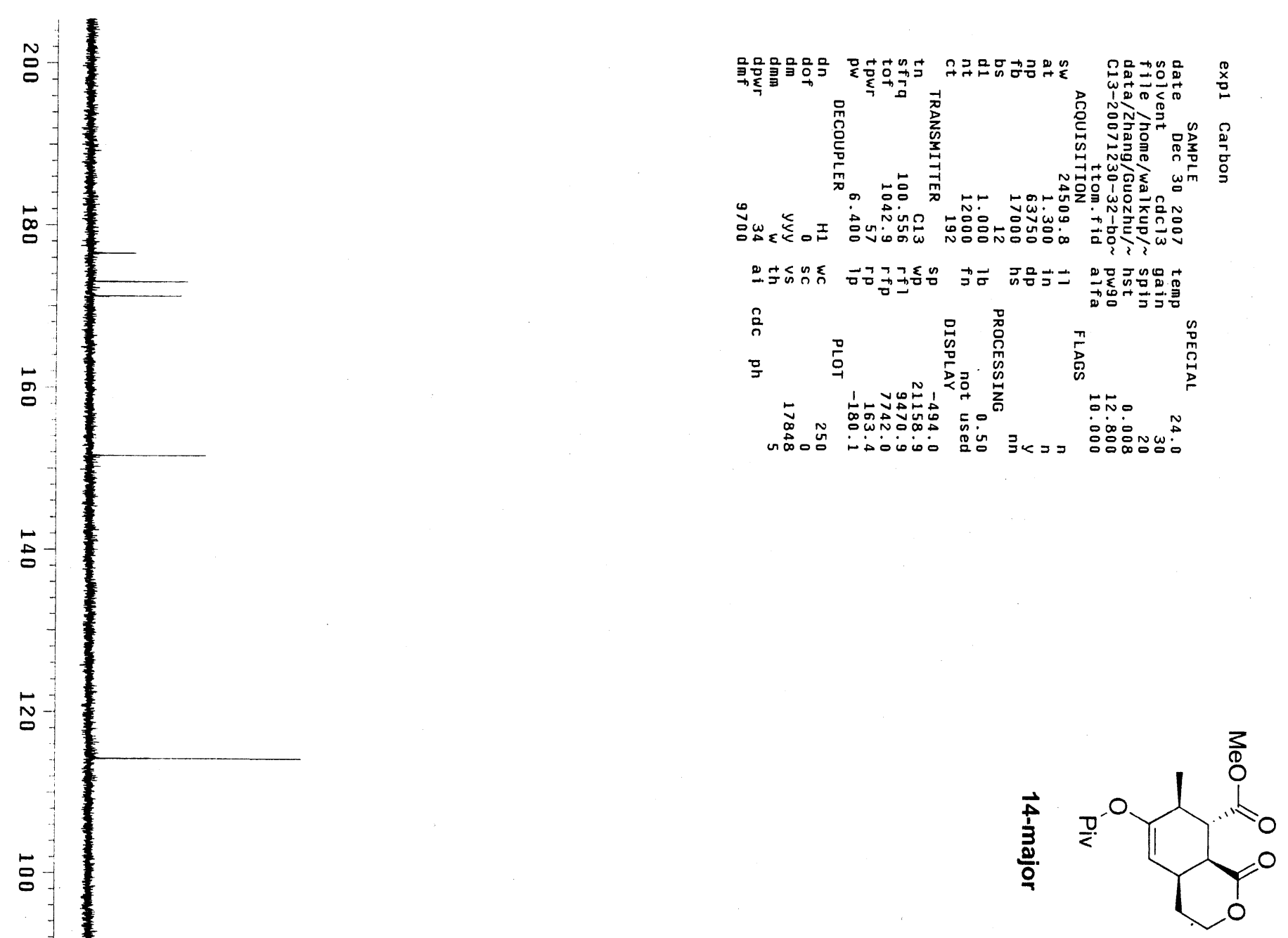


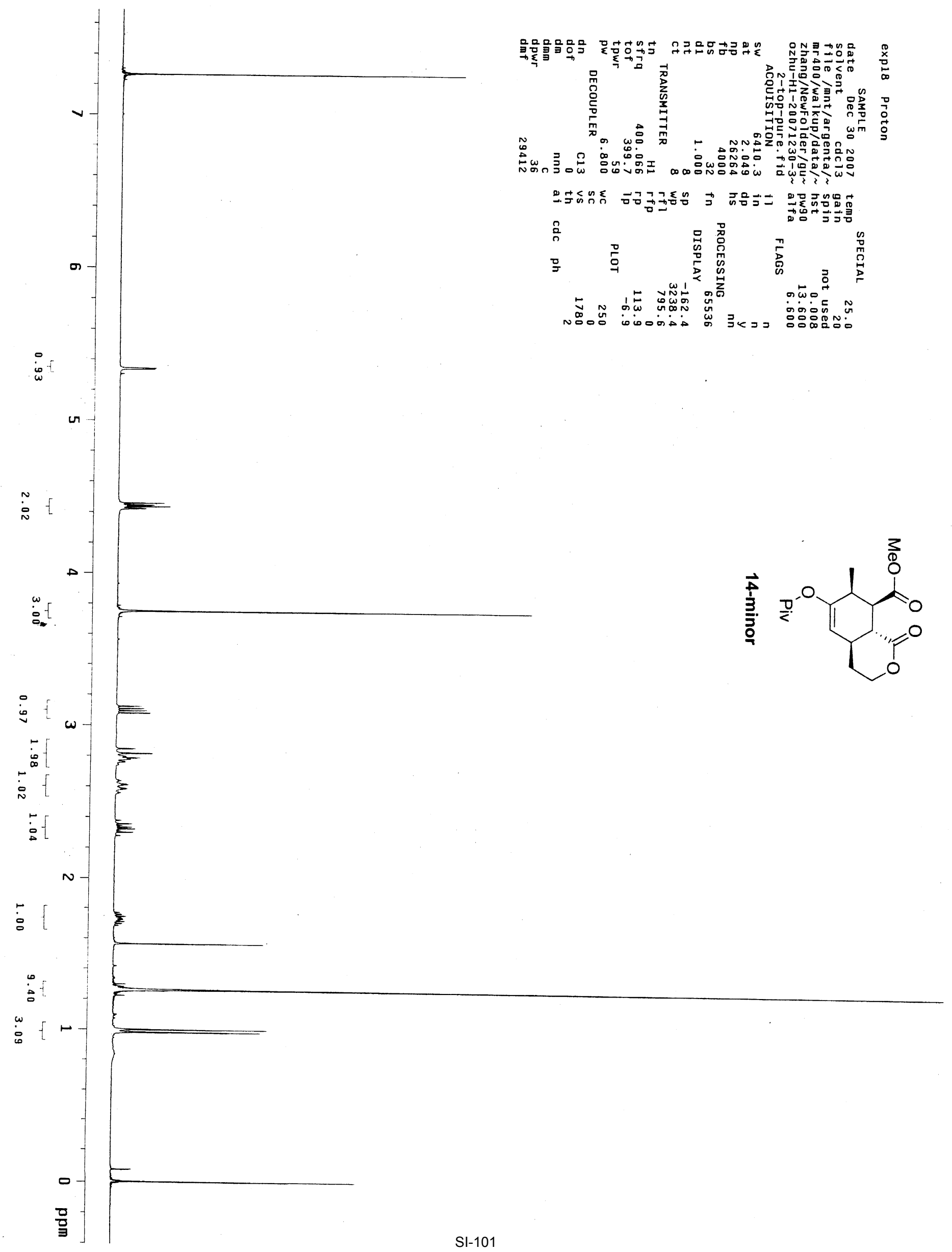



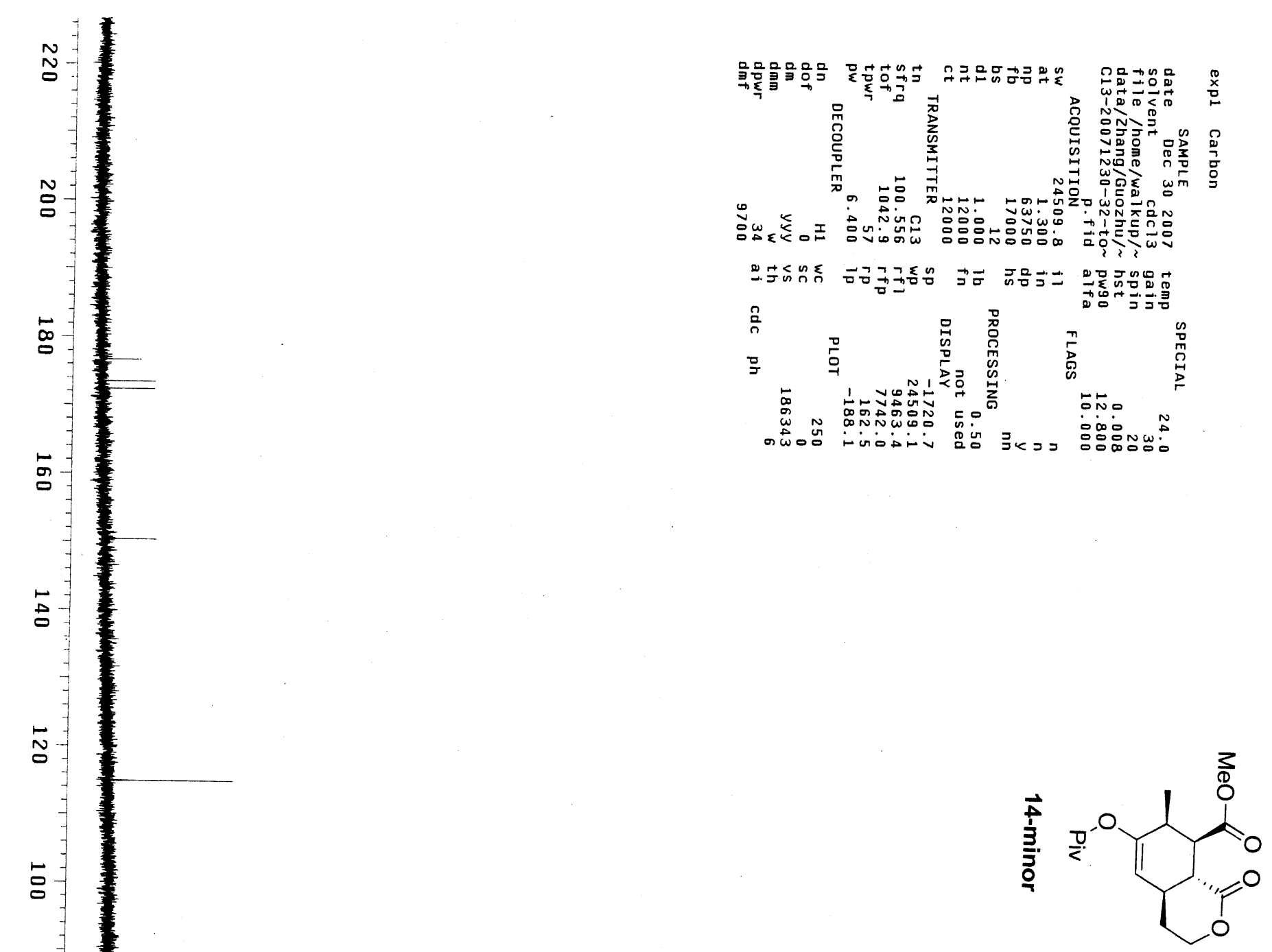